University of Louisville

ThinkIR: The University of Louisville's Institutional Repository

Electronic Theses and Dissertations

$12-2019$

\title{
Coordination of airway protective behaviors and swallow: effects of afferent feedback and sex.
}

Alyssa D. Huff

University of Louisville

Follow this and additional works at: https://ir.library.louisville.edu/etd

Part of the Physiological Processes Commons

\section{Recommended Citation}

Huff, Alyssa D., "Coordination of airway protective behaviors and swallow: effects of afferent feedback and sex." (2019). Electronic Theses and Dissertations. Paper 3342.

https://doi.org/10.18297/etd/3342

This Doctoral Dissertation is brought to you for free and open access by ThinkIR: The University of Louisville's Institutional Repository. It has been accepted for inclusion in Electronic Theses and Dissertations by an authorized administrator of ThinkIR: The University of Louisville's Institutional Repository. This title appears here courtesy of the author, who has retained all other copyrights. For more information, please contact thinkir@louisville.edu. 
COORDINATION OF AIRWAY PROTECTIVE BEHAVIORS AND SWALLOW: EFFECTS OF AFFERENT FEEDBACK AND SEX

By

Alyssa D. Huff

B.S.- Western Kentucky University, 2015

M.S.- University of Louisville, 2018

\begin{abstract}
A Dissertation
Submitted to the Faculty of the School of Medicine of the University of Louisville in Partial Fulfillment of the Requirements for the Degree of
\end{abstract}

Doctor of Philosophy in Physiology and Biophysics

Department of Physiology University of Louisville Louisville, Kentucky

December 2019 
(C) 2019

Alyssa D. Huff

All rights reserved 



\section{COORDINATION OF AIRWAY PROTECTIVE BEHAVIORS AND SWALLOW: EFFECTS OF AFFERENT FEEDBACK AND SEX}

\section{By}

Alyssa D. Huff

B.S.- Western Kentucky University, 2015

M.S.- University of Louisville, 2018

A Dissertation Approved on

August 14, 2019

by the following Dissertation Committee:

Teresa Pitts, Ph.D., Dissertation Advisor

Dale A. Schuschke, Ph.D., Dissertation Co-Advisor

Dena Howland, Ph.D.

Nicholas Mellen, Ph.D.

Amanda Jo LeBlanc, Ph.D.

Stuart K. Williams, Ph.D. 


\section{DEDICATION}

To my mom and dad.

For never giving up on me and

ALWAYS encouraging me. 


\section{ACKNOWLEDGMENTS}

First and foremost, I would like to thank Dr. Pitts for her mentorship and support over the past four years. What a journey, physically and mentally, we have been on. Dr. Howland, thank you for always being available to give histology advice and overall support. Dr. Mellen, thank you for spending all of your free time teaching me your SSRH preparation and your determination and dedication to stimulate swallow for 3 years! I'd like to thank Dr. Schuschke, Dr. LeBlanc and Dr. Williams for their guidance and encouragement as members of my thesis committee. I would like to thank Mitch, for your friendship, constant support, corny dad jokes and willingness to listen to all my comments. Kim, thank you for your editorial support throughout this process and to Wilbur for always helping me with anything and everything. Thank you Yun for all your efforts in histology.

Additionally, I would like to thank my family for their constant support and willingness to listen to anything I had to say, good or bad, at any time of day or night. Mom, thank you for enstilling in me that I can do anything I put my mind to. Dad, thank you for making science and education a priority in my life early on (eventhough it was engineering and not biological science). Amanda, thank you for paving the way through the $\mathrm{PhD}$ process and preparing expectations.

I cannot go without thanking all of my sweet bestfriends who have given me more love and support in my life than anyone could understand. You all are amazing women placed in my life to help me realize my potential. The pen is mightier than the sword. 


\title{
ABSTRACT \\ COORDINATION OF AIRWAY PROTECTIVE BEHAVIORS AND SWALLOW: EFFECTS OF AFFERENT FEEDBACK AND SEX
}

\begin{abstract}
Alyssa D. Huff
August 14, 2019

This dissertation represents a series of studies describing mechanisms related to breathing, upper airway behaviors and their coordination in man and animal. Chapter two transformed the cough swallow aspiration protocol from the cat (previous work) to the human introducing a new strategy, volume targeting, in swallow breathing coordination. Chapter three evaluated swallow breathing coordination at increasing altitudes. As respiratory drive altered due to hypoxia and hypocapnia, swallow breathing coordination shifted toward inspiration occurring during the transition from inspiration and expiration. The collection of the two previous studies led to development of an animal model to evaluate volume targeting and mechanisms involved in this strategy. Chapter four highlights presence of vagal spinal feedback on breathing characteristics and chapter five the same for swallow behavior and swallow breathing coordination. Chapter four and five also introduce sex differences in breathing and swallow breathing coordination when vagal and spinal balance is perturbed. In conclusion, this work has furthered the knowledge of swallow breathing coordination and suggested mechanisms responsible for these behaviors. Describing basic swallow parameters in human could lead to potential detection of pathologic changes in the upper airway as well as further the understanding
\end{abstract}


of pulmonary complications such as aspiration pneumonia. The influence of the thoracic cavity spinal feedback could lead to new therapeutic techniques for breathing, swallow and their coordination in spinal cord injured patients. 
TABLE OF CONTENTS

PAGE

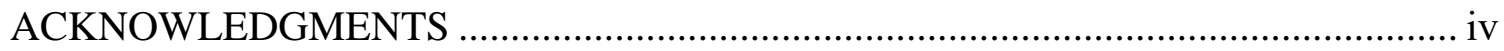

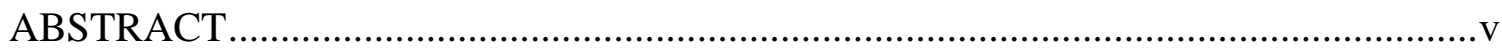

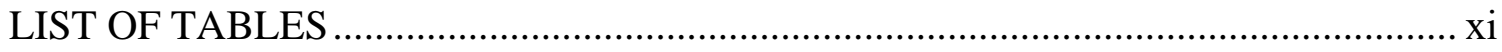

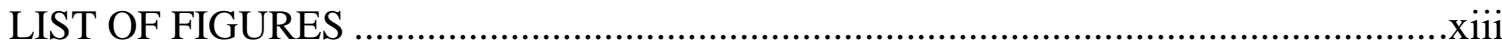

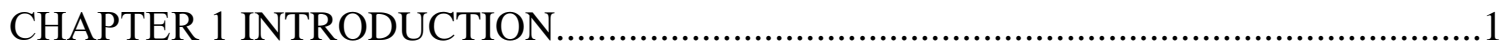

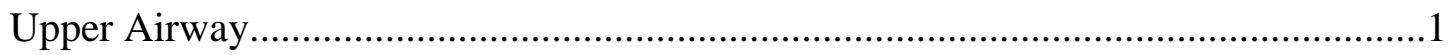

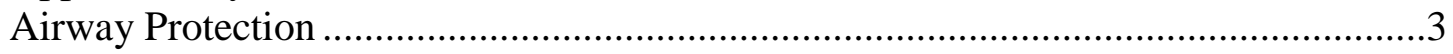

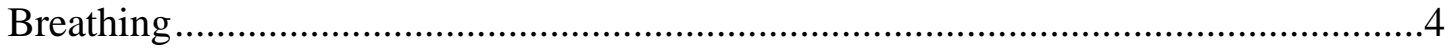

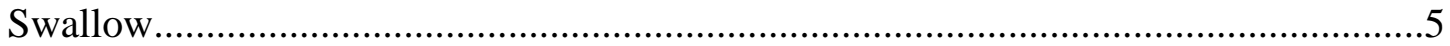

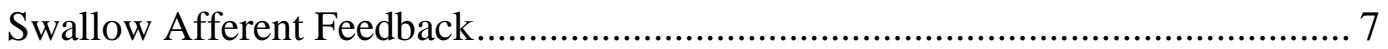

Swallow-Breathing Coordination ....................................................................... 8

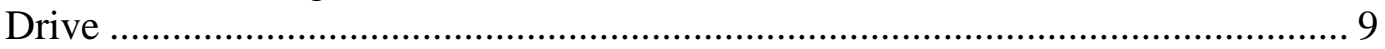

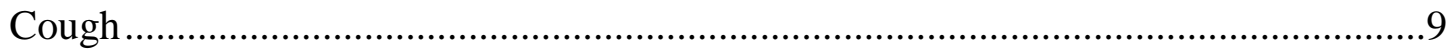

Coordination of Cough and Swallow................................................................11

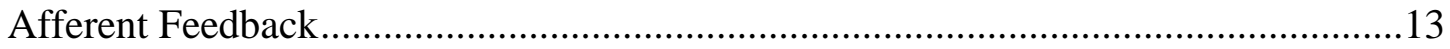

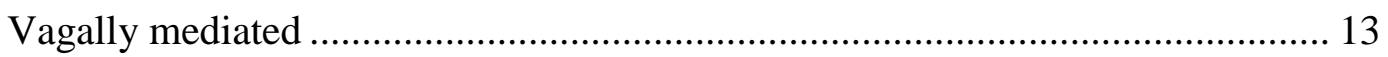

Spinally mediated ....................................................................................... 16

CHAPTER 2 STRATEGIES FOR THE INTEGRATION OF COUGH AND SWALLOW TO MAINTAIN AIRWAY PROTECTION IN HUMANS ………………………..........19

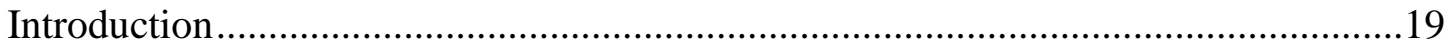

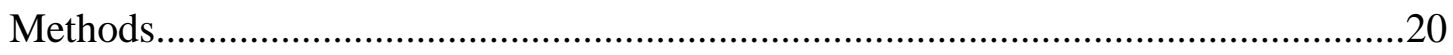

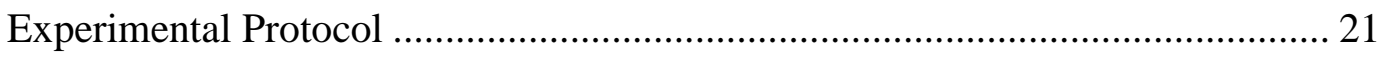

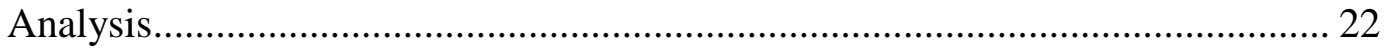

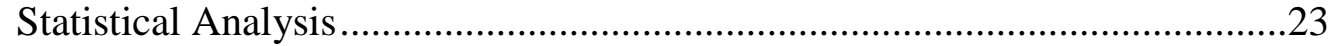

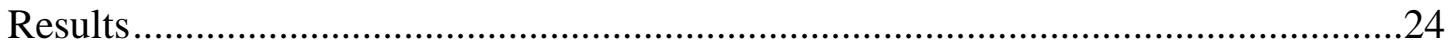

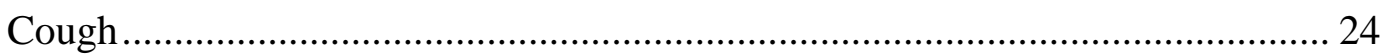

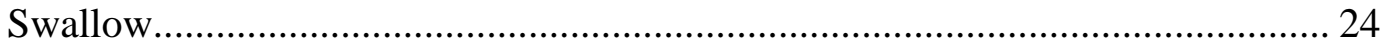

Cough and respiratory phase .................................................................... 25

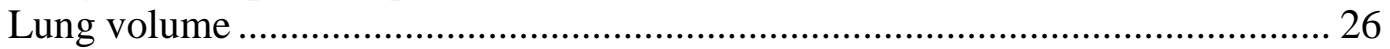

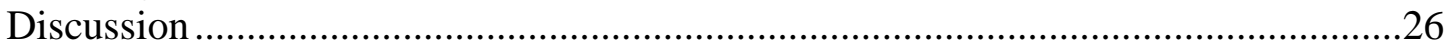

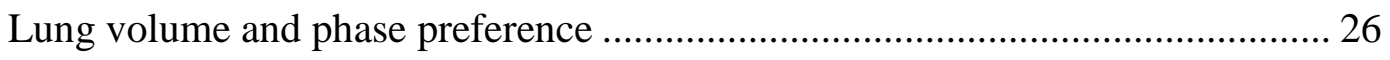

Swallows during the compression phase of cough ............................................ 29

Apnea Duration ..................................................................................... 29

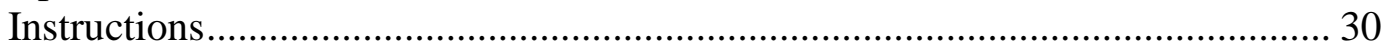


Conclusion

CHAPTER 3 SWALLOW-BREATHING COORDINATION DURING INCREMENTAL

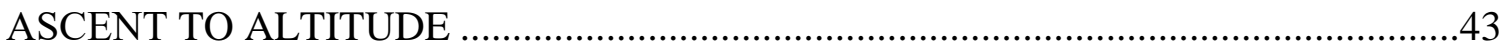

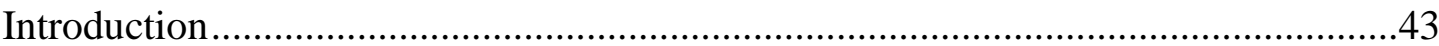

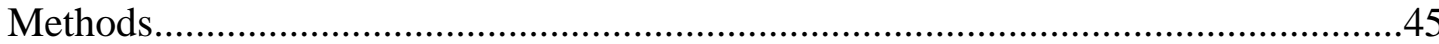

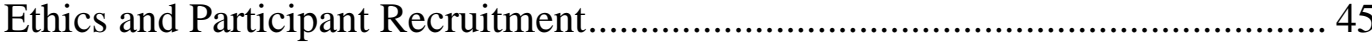

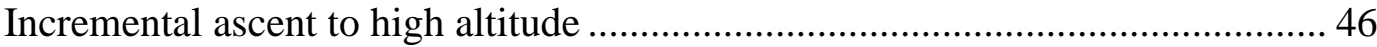

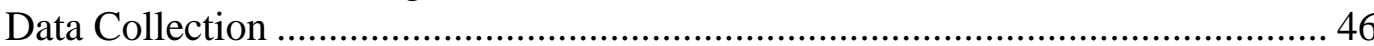

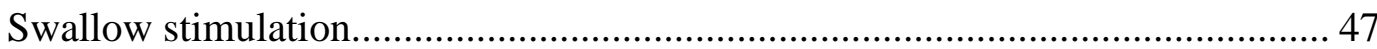

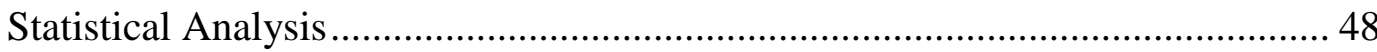

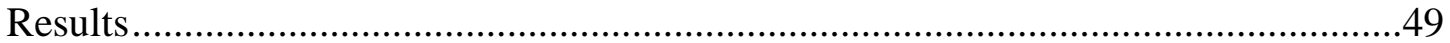

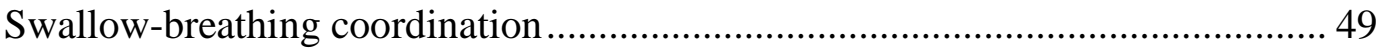

Change of swallow motor pattern with increasing altitude ............................ 50

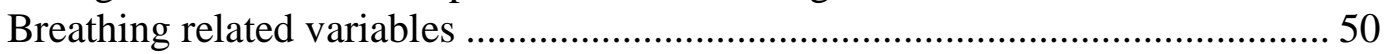

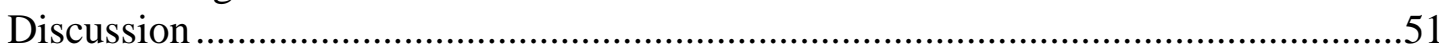

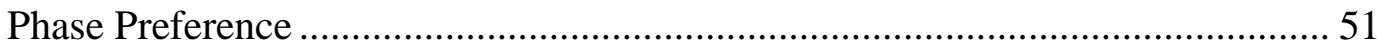

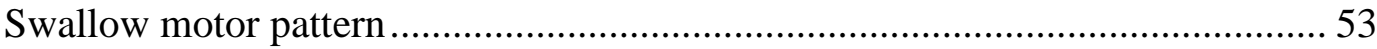

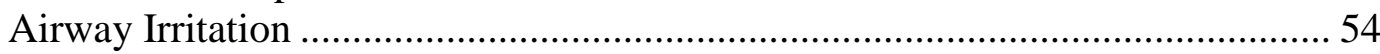

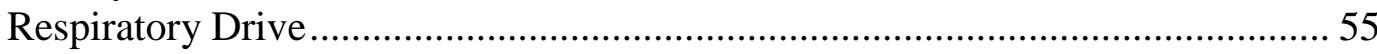

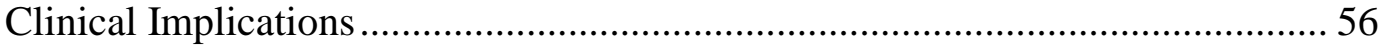

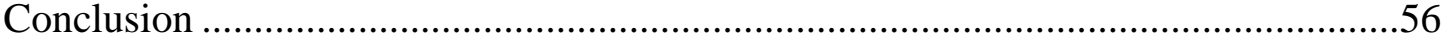

CHAPTER 4 SEX SPECIFIC VAGAL AND SPINAL MODULATION OF BREATHING

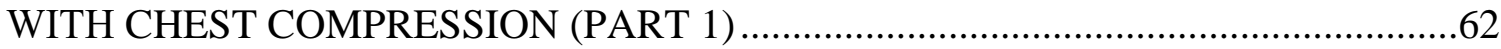

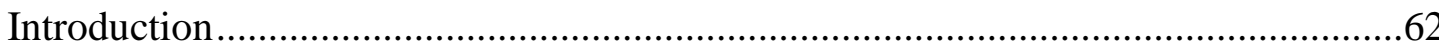

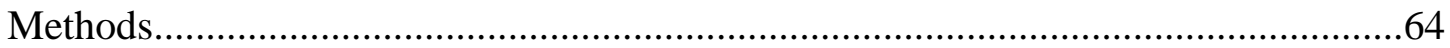

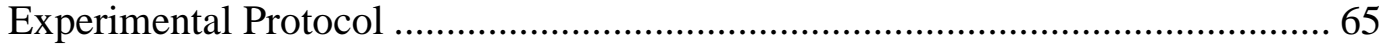

Removal/reduction of vagal feedback ....................................................66

Reduction of spinal feedback .............................................................67

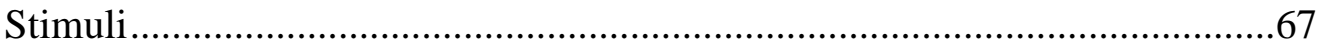

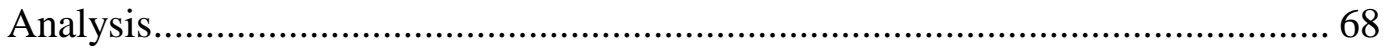

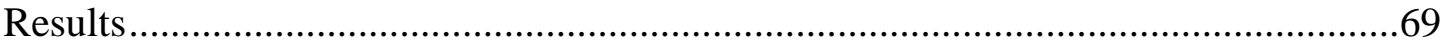

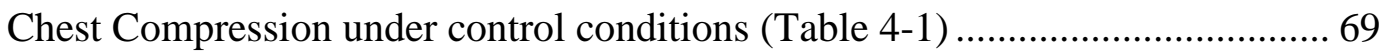

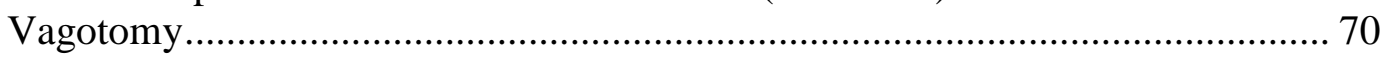

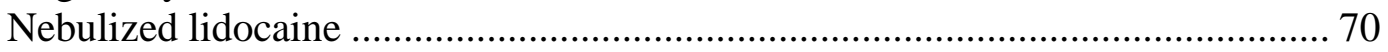

Altered afferent feedback by pleural administration of lidocaine ...................... 70

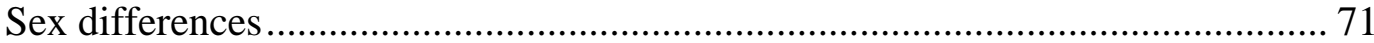

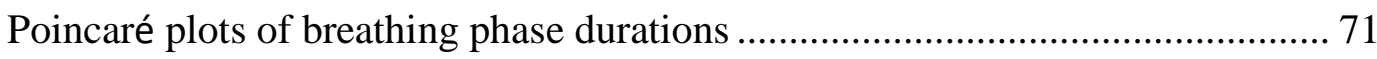

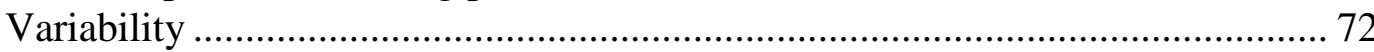

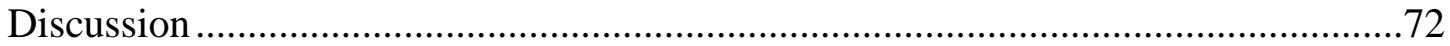

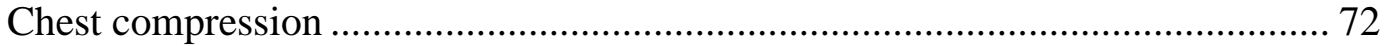

Yield: a novel description of diaphragm activity during early expiration ............ 73

Laryngeal contribution to breathing (E1) ...................................................... 76 


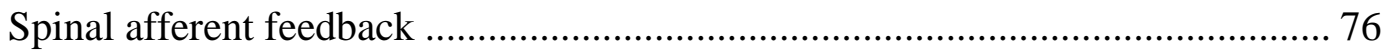

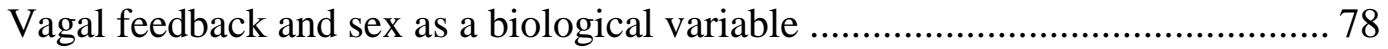

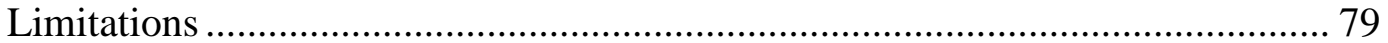

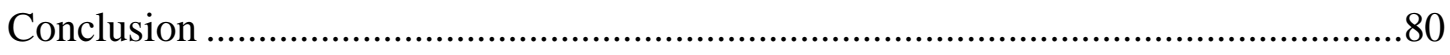

CHAPTER 5 SEX SPECIFIC VAGAL AND SPINAL MODULATION OF SWALLOW

AND ITS COORDINATION WITH BREATHING (PART 2) ......................................91

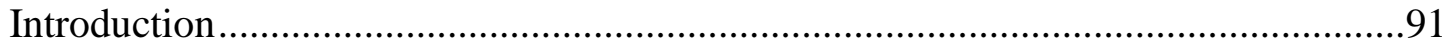

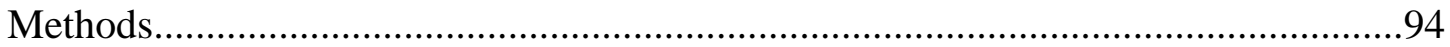

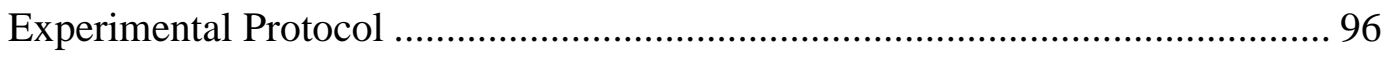

Removal/reduction of vagal feedback ………………..................................96

Reduction of spinal feedback ........................................................................97

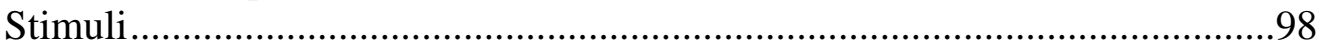

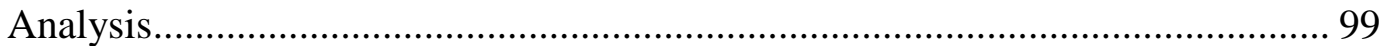

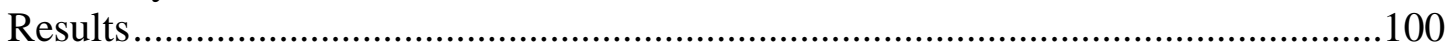

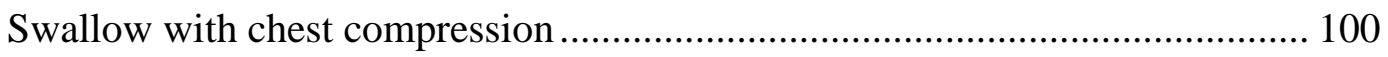

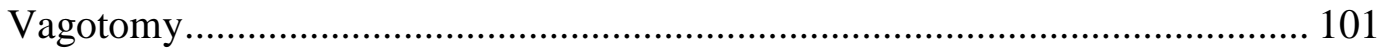

Local anesthesia of PSRs via lidocaine inhalation .......................................... 102

Local anesthesia of pleural afferents via lidocaine injection ............................... 102

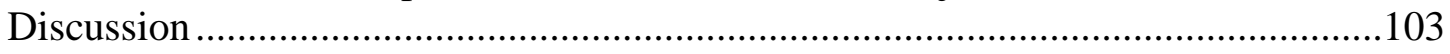

Sex differences in swallow-breathing coordination............................................ 103

Upper airway amplitude changes during swallow due to vagal and spinal feedback

105

Swallow duration relies on both PSR and spinal feedback................................. 106

EMG amplitude and duration are not correlated................................................ 106

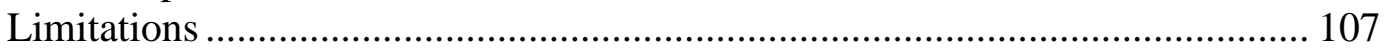

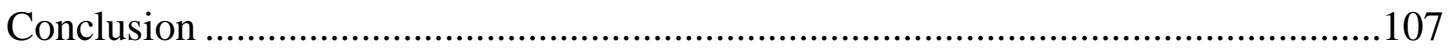

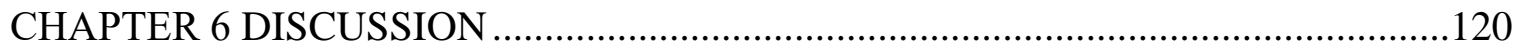

Phase Preference versus Volume Targeting ............................................................120

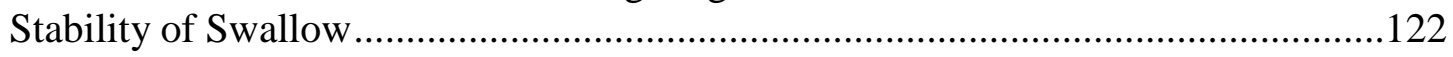

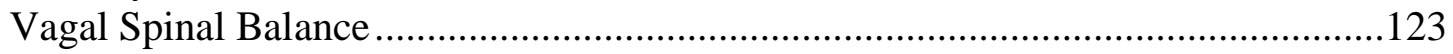

Sex Differences in Breathing and Swallow Coordination .........................................124

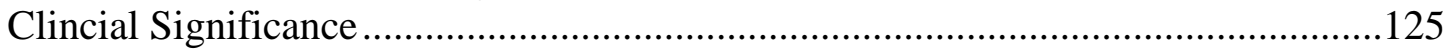

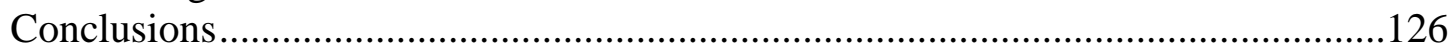

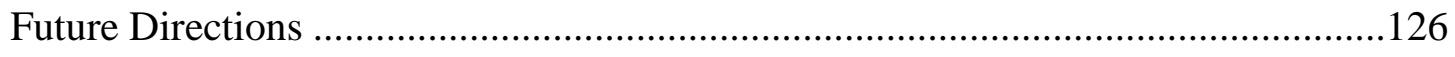

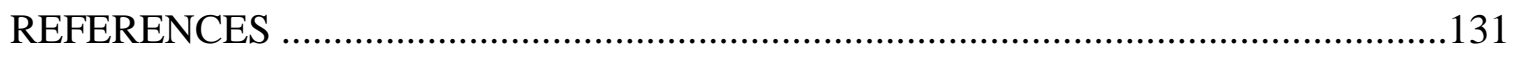

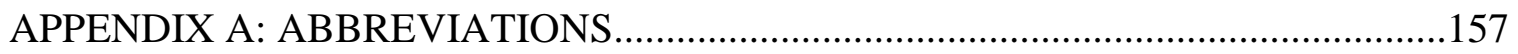

APPENDIX B: LUNG LICENSE AGREEMENT ........................................................

APPENDIX C: RESPIRATORY PHYSIOLOGY AND NEUROBIOLOGY LICENSE

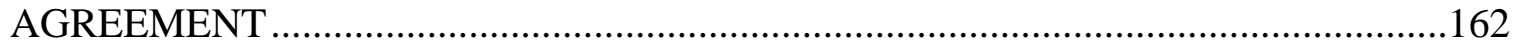


CURRICULUM VITAE. .165 


\section{LIST OF TABLES}

TABLE

PAGE

2-1. Means, standard deviations (SD), and p-value comparing cough protocol to combined stimuli protocol as well as swallow stimuli protocol to combined stimuli protocol for all measurements made in this study.

$\underline{3-1 .}$ Percent of swallow occurrence during breathing across the three levels of ascent ... 57

3-2. Means, standard deviations (SD), and $p$-values comparing ventilatory, cardiac and acclimation values, as well as saliva and water swallows at the three different elevations are shown in this table.

4-1. Means, standard deviation (SD), $p$-values and direction of change for breathing parameters during control and chest compression conditions are shown below for both male and female.

4-2. Means, standard deviation (SD), p-values and direction of change for breathing parameters during control and feedback modulation conditions are shown below for both male and female.

4-3. Means, standard deviation (SD), $\mathrm{p}$-values and direction of change for breathing parameters during chest compression and feedback modulation conditions are shown below for both male and female.

5-1. Means, standard deviation (SD), $p$-values and direction of change for swallow parameters during control and chest compression conditions are listed for both male and female groups.

5-2. Number of swallows during each phase of breathing for control and chest compression conditions are listed for both male and female groups.

5-3. Means, standard deviation (SD), $p$-values and direction of change for swallow parameters during control and feedback modulation conditions are listed for both male and female groups. 
5-4. Number of swallows during each phase of breathing during control and feedback modulation conditions are listed for both male and female groups.................... 112

5-5. Means, standard deviation (SD), $p$-values and direction of change for swallow parameters during chest compression and feedback modulation conditions are listed for both male and female groups............................................................ 114

5-6. Number of swallows during each phase of during chest compression and feedback modulation conditions are listed for both male and female groups.................... 116 


\section{LIST OF FIGURES}

FIGURE

PAGE

2-1. Cough epochs can be characterized using airflow and oblique surface EMG activity.

2-2. Examples of swallow occurring during each phase of cough. ............................... 36

2-3. Swallows occurring during the inspiratory phase of cough. ................................. 38

2-4. Abdominal EMG depression during compression phases which include swallow... 39

2-5. All swallows graphed on percent VC by breathing and cough respiratory phase..... 41

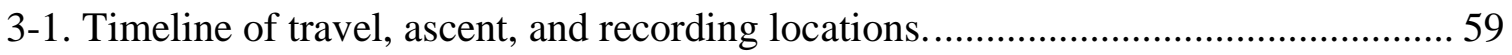

3-2. Example of submental sEMG and airflow from the same participant from Calgary $(1,045 \mathrm{~m})$ and Pheriche $(4,371 \mathrm{~m})$ during the water swallow protocol. ................. 60

3-3. Scatter plot of duration measures (pre-swallow, submental and post-swallow) for each subjects across the recording locations for the saliva (A) and water (B) swallow tasks.

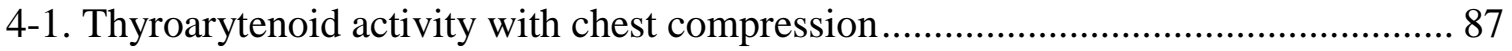

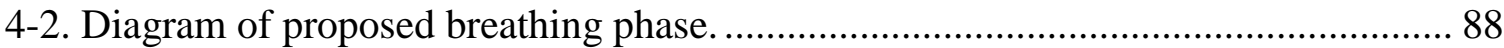

4-3. Poincaré plots demonstrating difference in expiration duration variability across all three afferent feedback interventions in both male and female. ........................ 89

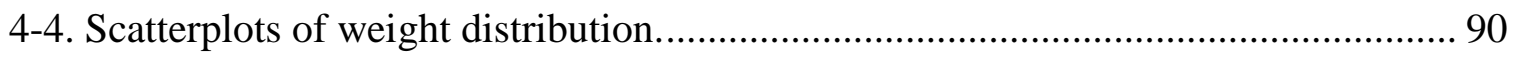

5-1. Experimental perturbations shifted swallow-breathing coordination in females.... 118

5-2. Representative EMG traces of swallow activity before and after afferent feedback

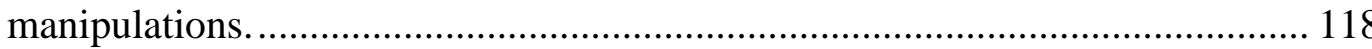


6-1. Presence of CTB in the cervical and thoracic DRG after pleural space CTB

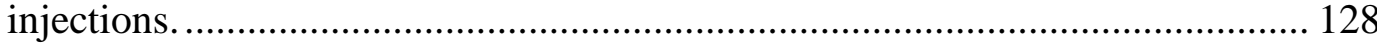

6-2. Stimulation of ficitive swallowin the sagittal section....................................... 129 


\section{CHAPTER 1 INTRODUCTION}

Swallow and breathing share anatomical structures. Coordination of these two behaviors is physiologically necessary in order to maintain a patent airway. Afferent feedback from vagal and spinal pathways function to maintain this coordination. This vagal spinal balance across human and animal will be discussed throughout this text.

\section{Upper Airway}

Upper airway muscle activity have both respiratory and non-respiratory (mastication, deglutition, communicative and defensive) functions (Erik Van Lunteren, 1988).

Anatomically the upper airway consists of the pharynx, larynx and extrathoracic portion of the trachea. The pharynx can further be categorized into nasopharynx, oropharynx and laryngopharynx (Sant'Ambrogio, Tsubone, \& Sant'Ambrogio, 1995). Muscles of the oropharynx include: geniohyoid, innervated by the hypoglossal nerve; mylohyoid, innervated by the trigeminal nerve; and hypoglossus, stylohyoid and digastric all innervated by the facial nerve (Erik Van Lunteren, 1988). Laryngopharyx muscles include: thyrohyoid innervated by the first cervical nerve via hypoglossal, and sternohyoid and omohyoid innervated by the ansa cervicalis (Erik Van Lunteren, 1988). 
Laryngeal muscles are classified as intrinsic or extrinsic, but more specifically adductors, which act to close the vocal folds, or abductors which open the vocal folds. The posterior crycoarytenoid (PCA), an inspiratory phasic muscle abducts the vocal folds ensuring airway patency and is innervated by the recurrent laryngeal nerve (RLN) branch of the vagus nerve. Thyroarytenoid, an expiratory phasic muscle, adducts the vocal folds, which regulates the rate of airflow during expiration and is innervated by the RLN as well as the superior laryngeal nerve (SLN) (Nasri, Beizai, Ye, Sercarz, Kim, \& Berke, 1997). Thyropharyngeus also known as the inferior pharyngeal constrictor muscle, is expiratory phasic in the cat and inspiratory phasic in the rat (Pitts, Rose, Mortensen, Poliacek, Sapienza, Lindsey, Morris, Davenport, \& Bolser, 2013b), and is innervated by the vagus. All laryngeal efferent information, except for cricothyroid, is supplied by the RLN and all afferent information is supplied by SLN (Oommen P Mathew, 1988). The motoneurons that drive intrinsic laryngeal muscles and pharyngeal muscles extend throughout the nucleus ambiguus (NA) with its most rostral portion at the reticular formation and most caudal at the start of the spinal cord (Iscoe, 1988; Nomura \& Mizuno, 1982; Yoshida, Miyazaki, Hirano, Shin, Totoki, \& Kanaseki, 1980, 1981).

Motoneurons and muscles of the upper airway can be affected by three external factors: anesthesia, arousal and posture. An increase in the depth of anesthesia decreases, and potentially abolishes the activity of upper airway muscles during expiration (Rujdomin, 1966), while inspiratory muscles of the upper airway are less sensitive to anesthesia but changes can still occur (Iscoe, 1988). It has been shown that topical application of anesthesia onto pharyngeal and laryngeal mucosa block both slowly and rapidly adapting receptors (Camporesi, Mortola, Sant'Ambrogio, \& Sant'Ambrogio, 
1979). The muscles of the upper airway have different responses during different behavioral or arousal states during REM sleep and awake states (Iscoe, 1988). Posture of the animal, whether in supine or prone can affect the behavior of the upper airway (Iscoe, 1988).

\section{Airway Protection}

Maintenance of airway protection while simultaneously maintaining homeostasis is observed in vertebrates in one of two ways involving the alimentary and respiratory systems (Mathew, 1988). First, separation of the two systems resulting in independent respiration and feeding processes. The other, coordination of swallow and breathing where both systems work synergistically to maintain homeostasis and proper function of each system (Mathew, 1988).

Aspiration reflex is an airway protective reflex that consists of a series of diaphragm contractions in the absence of abdominal activity. This reflex occurs when nasopharyngeal mucosa is stimulated (Korpáš \& Tomori, 1979; Tomori \& Widdicombe, 1969; Widdicombe, 2011) or foreign material enters into the trachea instead of the esophagus. Pitts et al. (2013b) described the production of cough and swallow, in response to aspiration, as a meta-behavior due to the alterations in the gain of these behaviors. This change in gain describes allostasis, the ability to maintain stability through predictable and unpredictable changes within the system (Fibla, Bernardet, \& Verschure, 2010).

Laryngeal expiration reflex is an airway protective response that prevents the entrance of foreign material into the lower airways. It is characterized by a quick, large amplitude expiratory effort with coordinated laryngeal closure and opening of the vocal 
folds (Shannon, Baekey, Morris, \& Lindsey, 2005). An expiration reflex is different from cough due to the absence of a large inspiration proceeding the expiratory effort (Korpas \& Jakus, 2000).

\section{Breathing}

The way in which breathing is analyzed or observed shapes the way we define the phases of this behvaior. Analysis of breathing from airflow traces corresponds with two phases: inspiration and expiration (Huff, Reed, Smith, Brown, Ovechkin, \& Pitts, 2018b; Wheeler Hegland, Huber, Pitts, Davenport, \& Sapienza, 2011b). Analysis of breathing from inspiratory muscles such as the diaphragm results in three phases: inspiration, the transition from inspiration to expiration and expiration. When looking at inspiratory or expiratory related neurons phase of breathing are termed inspiration, post inspiration and expiration (Anderson, Garcia, Baertsch, Pollak, Bloom, Wei, Rai, \& Ramirez, 2016). In preps where laryngeal, phrenic and abdominal signal is present breathing is defined as inspiration, early expiration (E1) and late or active expiration (E2) (Bianchi \& Gestreau, 2009).

Respiratory neurons necessary for control of breathing are located in the pons and medulla. The core circuits of respiratory rhythm generation are along the medullary ventral respiratory column (VRC) which include (rostral to caudal) RTN/pFRG, Bötzinger complex, preBötzinger complex, rostral and caudal ventral respiratory group (Ramirez \& Baertsch, 2018). The respiratory central pattern generator (rCPG) is responsible for the control of two cohort of motoneurons in order to ensure proper respiration: inspiratory muscles, such as the diaphragm, whose motoneurons are located 
in the spinal cord (Iscoe, 1998; Monteau \& Hilaire, 1991) and the upper airway motoneurons which control airflow located in the brainstem (Bartlett Jr, 1989).

The Kölliker Fuse nucleus is important in the adaptation of breathing in response to other behaviors (Dutschmann \& Dick, 2012) as well as the transition from inspiration to expiration due to phase spanning neurons that fire during the transition from inspiration to expiration (Cohen \& Shaw, 2004; Dick, Shannon, Lindsey, Nuding, Segers, Baekey, \& Morris, 2008; Ezure \& Tanaka, 2006). Kölliker Fuse has descending fibers that project onto respiratory areas in the medulla, NTS and NA (Dobbins \& Feldman, 1994; Ellenberger \& Feldman, 1990; Herbert, Moga, \& Saper, 1990) trigeminal, facial and hypoglossal motoneurons (Fay \& Norgren, 1997; Herbert, Moga, \& Saper, 1990; Rikard-Bell, Bystrzycka, \& Nail, 1984; Takada, Itoh, Yasui, Mitani, Nomura, \& Mizuno, 1984); as well as cervical phrenic motoneurons (Fulwiler \& Saper, 1984; Rikard-Bell, Bystrzycka, \& Nail, 1984) and spinal motoneurons for intercostal and abdominal muscles in the spinal cord (Rikard-Bell, Bystrzycka, \& Nail, 1985). Kölliker Fuse's vast connection to motoneurons involved in breathing, swallow and cough suggest it is critical in the modulation of the CPGs involved in these behaviors.

\section{Swallow}

Swallow involves the coordination of more than 26 pairs of muscles and five cranial nerves to ensure proper food breakdown (oral phase), food or liquid bolus transfer (pharyngeal and esophageal phase) and overall safe swallow (Barlow, 2009). The most common physiologic stimulation of swallow is insertion of liquid into the oral cavity stimulating the pharyngeal wall. Swallow is also initiated by natural stimulation of the epiglottis, tongue, soft palate and cranial part of the pharynx (Miller, 1982a; Nail, 
Sterling, \& Widdicombe, 1972; Widdicombe, 2011) as well as electrical stimulation of the SLN. Swallow is mediated primarily by the glossopharyngeal as well as the trigeminal, superior laryngeal and hypoglossal nerves (Widdicombe, 1988).

There are three phases and four stages that make up the process of swallow. The oral phase has two stages: 1) Preparatory Stage in which food is broken down and formed into a bolus of optimal shape and size (Mathew, 1988). This stage is under voluntary control. 2) Propulsion Stage in which the tongue moves the bolus to the back of the pharyngeal wall causing stimulation of mechanoreceptors and thus triggers peristalsis. This peristalsis marks the transition to the pharyngeal phase. During the pharyngeal phase, the pharynx moves anteriorly and cranially (Mathew, 1988; Negus, 1942; Shelton Jr, Bosma, \& Sheets, 1960), the larynx elevates and is pulled forward under the tongue, the upper esophageal sphincter (UES) relaxes, and the laryngeal adductors close the larynx which leads to protection of the airway by closure of the vocal folds (Ardran \& Kemp, 1952; Mathew, 1988). Like the preparatory stage, the propulsion stage is under voluntary control. Once the bolus has traveled through the pharyngeal cavity, passing the UES and entering the esophagus, the two stages of the pharyngeal phase are completed and the esophageal phase begins. The esophageal phase does not end until the bolus reaches the lower esophageal sphincter and enters into the stomach (Jean, 2001a). There is less focused on the behavior and the mechanism of the esophageal phase of swallow and will not discuss further.

Neural recordings in many species have identified two main locations in the brainstem where swallow neurons are located. The dorsal swallow group (DSG) located in the dorsal medulla within the NTS and adjacent reticular formation; and the ventral 
swallow group (VSG) located in the ventral lateral medulla just above the NA (Jean, 2001a). The DSG contains the generator neurons which process convergent information, both cortical and peripheral, leading to triggering, shaping and sequential timing within swallow (Jean, 2001a). An intact DSG within the NTS is required for both peripherally and cortically induced pharyngeal swallow (Car, 1979; Car, Jean, \& Roman, 1975;

Kessler \& Jean, 1985; Wang \& Bieger, 1991). The presence of the bolus or the distention of the pharyngeal cavity stimulates slowly adapting receptors in the pharyngeal and laryngeal mucosa (Tomori \& Widdicombe, 1969) comprised of A $\alpha$ and A $\delta$ afferent fibers of the SLN, which then terminate onto the NTS. The VSG contains switch neurons which are involved in distribution of drive to the various swallow motoneurons pools (Jean, 2001a).

Kölliker Fuse acts as a descending inhibitory input gating sequential pharyngeal swallow generation (Bautista \& Dutschmann, 2014). Inhibition of the Kölliker Fuse results in an increase in spontaneous pharyngeal swallows (Bonis, Neumueller, Marshall, Krause, Qian, Pan, Hodges, \& Forster, 2011b; Bonis, Neumueller, Krause, Pan, Hodges, \& Forster, 2013). Jean (2001a) describes these pontine neurons as sensory relay neurons that provide information from the oropharyngeal receptors to the higher central nervous centers which are not a part of the swallow CPG.

\section{$\underline{\text { Swallow Afferent Feedback }}$}

Motor output of swallow is modified by afferent feedback from various sensory characteristics of the bolus such as volume, viscosity, taste, temperature and size (Troche, Brandimore, Godoy, \& Hegland, 2014). These modifications include: UES and swallow apnea duration, number of sequential swallows, initiation and timing of pharyngeal 
phase, laryngeal closure, lung volume, and oropharyngeal pressure (Troche, Brandimore, Godoy, \& Hegland, 2014) as well as EMG duration and amplitude (Jean, 2001a). Though swallow is organized centrally it is modified by afferent information thought to be controlled by peripheral feedback mechanisms (Jean, 2001a).

Laryngeal, pharyngeal and esophageal afferent fibers project to the subnuclei of the NTS (Jean, 2001a). Application of three percent xylocaine to the oropharyngeal mucosa resulted in an absence in swallow response and inactivation of mucosal receptors while having no effect on oropharyngeal muscles (Sumi, 1963b). These receptors are thought to be slowly adapting mechanoreceptors located throughout the oral, pharyngeal and laryngeal mucosa which are inhibited by topical anesthetics (MÅrnsson \& Sandberg, 1974).

\section{$\underline{\text { Swallow-Breathing Coordination }}$}

Neural coordination of swallow and breathing result from neurons in the NTS modulated by afferent information via oropharyngeal mucosa and muscles (Sumi, 1963b). Neurons in the NA and hypoglossal nuclei control swallow and respiratory neurons (Sumi, 1963b). Respiratory neurons in the reticular formation switch to bursting activity during swallow, becoming swallow-related neurons (Sumi, 1963a) while other respiratory neurons become silent (Mathew, 1988). Swallow CPG in the NTS is densely connected to Kölliker Fuse in the dorsolateral pons (Bautista \& Dutschmann, 2014). Postinspiratory neurons in the Kölliker Fuse (Bonis, Neumueller, Marshall, Krause, Qian, Pan, Hodges, \& Forster, 2011b; Bonis, Neumueller, Krause, Pan, Hodges, \& Forster, 2013; Oku \& Dick, 1992) as well as a balanced synaptic interaction along this NTS/ Kölliker Fuse neuroaxis is needed for swallow breathing coordination (Herbert, Moga, \& 
Saper, 1990; Song, Xu, Wang, MacDonald, \& Poon, 2011). Hyperexcitability of the Kölliker Fuse results in a delay in sequential pharyngeal swallows due to heightened pharyngo-glottal closure reflex while hyperexcitability of the NTS results in excessive spontaneous pharyngeal swallows, suppressing respiratory activity (Bautista \& Dutschmann, 2014).

\section{Drive}

Motoneurons and interneurons may be involved in more than one activity such as swallow and breathing (Gestreau, Milano, Bianchi, \& Grélot, 1996; Pitts, Poliacek, Rose, Reed, Condrey, Tsai, Zhou, Davenport, \& Bolser, 2018; Saito, Ezure, \& Tanaka, 2002c) or swallow and mastication or swallow and chewing. During early states of asphyxia, respiratory-related muscles, also active during swallow, are recruited to increase respiratory effort in which respiratory drive exceeds swallow drive (Jean, 2001a). When drive for airway protection supersedes the drive for ventilation, laryngeal reflexes override ventilatory behaviors (Sasaki \& Buckwalter, 1984).

\section{Cough}

Cough is an airway protective behavior that removes foreign material from the airway, while apnea and bronchoconstriction stop further movement of foreign material into the airway (Korpáš \& Tomori, 1979). Cough can be initiated either reflexively due to response or irritation, or voluntarily via cortical command. Reflexive cough can be triggered mechanically by stimulating the larynx (Tatar, Sant' Ambrogio, \& Sant' Ambrogio, 1994) resulting in prolongation of expiration (Tatar, Sant' Ambrogio, \& Sant' Ambrogio, 1994), or moving more caudal to the tracheal bifurcation (Widdicombe, 1954). The cough reflex is elicited by the stimulation of rapidly adapting "irritant 
receptors" (Trenchard, 1977). In the presence of anesthesia laryngeal cough is depressed compared to tracheobronchial cough (Tatar, Sant' Ambrogio, \& Sant' Ambrogio, 1994).

There are three phases of cough: inspiration, compression and expiration. The inspiratory phase involves chest wall and laryngeal inspiratory muscles that draw air into the lungs until the targeted lung volume is reached. This leads to a compression phase where the laryngeal muscles activate glottal closure of the airway and activity of abdominal expiratory muscles build high pressure against the closed larynx. This leads to the expiratory phase where the built up of pressure turns into sheering forces when the closed airway opens, moving air from the lungs, with help from chest well and abdominal expiratory muscles, clearing the airway of any foreign material.

Slowly adapting receptors have an influence on the coordination of deep inspiration and expiration associated with cough (Tatar, Sant' Ambrogio, \& Sant' Ambrogio, 1994). Tatar (1994) performed laryngeal stimulation in the presence of reduced afferent feedback and found an increased inspiration and an absence of expiratory cough effort. An impairment of expiratory muscle recruitment and therefore development of cough would occur in the absence of stretch receptor feedback or reduction of afferent information (Tatar, Sant' Ambrogio, \& Sant' Ambrogio, 1994). These observations stemmed when Sant'Ambrogio et al. (1984) eliminated slowly adapting receptors resulting in an absence of cough from mechanical stimulation of the trachea. Bucher (1958) proposed the idea that pulmonary stretch receptors (PSR) fire more intensely during the inspiratory phase of cough resulting in increased inhibition to inspiration and a strengthen stimulation for the subsequent expiration. This observation of PSRs influence on cough was confirmed when slowly adapting PSRs were eliminated 
with $\mathrm{SO}_{2}$ decreasing the cough sensitivity and weakened expiratory efforts (Hanacek, Davies, \& Widdicombe, 1984).

Vagal afferents enter into the brainstem via the NTS (Kubin, Alheid, Zuperku, \& McCrimmon, 2006; Shannon, Baekey, Morris, \& Lindsey, 2005). The neural network of cough is known as Böt-VRG which is made up of: Bötzinger, preBötzinger and ventral respiratory group (Shannon, Baekey, Morris, \& Lindsey, 2005). This network is thought to be involved in breathing and rhythm regulation of cough (Baekey, Morris, Gestreau, Li, Lindsey, \& Shannon, 2001; Shannon, Baekey, Morris, \& Lindsey, 1996; Shannon, Baekey, Morris, Nuding, Segers, \& Lindsey, 2004a; Shannon, Baekey, Morris, \& Lindsey, 1998). The three phases of cough, as well as the magnitude of respiratory muscle activation for this behavior are controlled by neurons of the ventrolateral medulla, raphe nuclei and pons (Baekey, Morris, Gestreau, Li, Lindsey, \& Shannon, 2001; Baekey, Morris, Nuding, Segers, Lindsey, \& Shannon, 2003; O'Connor, Segers, Morris, Nuding, Pitts, Bolser, Davenport, \& Lindsey, 2012; Shannon, Baekey, Morris, Li, \& Lindsey, 2000; Shannon, Baekey, Morris, Nuding, Segers, \& Lindsey, 2004a; Shannon, Baekey, Morris, \& Lindsey, 1998; Shannon, Baekey, Morris, Nuding, Segers, \& Lindsey, 2004b). This network of neurons is capable of producing both cough and breathing behaviors and reconfigures in order to support both behaviors (Baekey, Morris, Gestreau, Li, Lindsey, \& Shannon, 2001; Shannon, Baekey, Morris, Li, \& Lindsey, 2000; Shannon, Baekey, Morris, \& Lindsey, 1998).

\section{Coordination of Cough and Swallow}

Smith-Hammond was the first to describe the relationship between cough and swallow in the stroke population $(2009 ; 2001)$. Pitts, et al $(2009 ; 2008 ; 2010)$ and 
Hegland, et al $(2014 ; 2014 ; 2016)$ examined this relationship in Parkinson's disease with Pitts et al. (2013b) being the first to study the coordination of cough and swallow using an aspiration protocol in the cat. This study found that $95 \%$ of swallows occurred during the E2 (late expiratory) phase of cough and this phase was significantly longer in duration than in swallow alone trials. Total swallow duration decreased, swallow amplitude increased, and the aspiration protocol elicited significantly more swallows (Pitts, Rose, Mortensen, Poliacek, Sapienza, Lindsey, Morris, Davenport, \& Bolser, 2013b). Huff et al. (2018b) modified this aspiration protocol to test the coordination of cough and swallow in healthy non-disabled human population. This study showed reliable integration of cough and swallow, an increase in cough epochs and an increase in inspiratory and compression phase duration during the combined stimuli protocol. The most interesting finding of this study was the participants' ability to swallow in any phase of cough, unlike the cat, to maintain a certain range of lung volume during eupnea and repetitive cough (Huff, Reed, Smith, Brown, Ovechkin, \& Pitts, 2018b). This indicated that volume targeting may play a larger role than phase preference. This study lead to speculation on the influence of vagal afferent feedback on cough-swallow-breathing regulation and coordination.

Cough and swallow behaviors share afferent feedback that are necessary for initiation and modification. These afferents project to sensory nuclei within the brainstem which then project to the behavioral control assembly where the cough and swallow CPGs interact and the appropriate motor output is sent via efferent motor neurons and the generation of the appropriate behavior is exerted (Troche, Brandimore, Godoy, \& Hegland, 2014). The pharyngeal branch of the hypoglossal nerve (Kitagawa, Nakagawa, 
Hasegawa, Iwakami, Shingai, Yamada, \& Iwata, 2009) and the internal branch of the SLN (Gestreau, Grélot, \& Bianchi, 2000; Jafari, Prince, Kim, \& Paydarfar, 2003; Jean, 2001a; Storey, 1968) is involved in the initiation of cough and swallow. Gestreau et al. (2000) showed this by direct stimulation of the SLN to produce fictive cough and at a much higher frequency, fictive swallow.

\section{Afferent Feedback}

\section{$\underline{\text { Vagally mediated }}$}

There are three types of lung sensory receptors whose afferent feedback travels via the vagus nerve. Slowly adapting receptors also known as pulmonary stretch receptors (PSRs) are responsible for the Hering-Breuer inflation reflex (Kubin, Alheid, Zuperku, \& McCrimmon, 2006). Rapidly adapting receptors are stimulated by rapid inflation/deflation of the lungs, mechanical simulants and irritant receptors resulting in excitatory responses such as augmented breaths (H.M. Coleridge, 1986; Widdicombe, 2003). Bronchopulmonary C fibers respond to chemical stimuli, temperature changes, and inflation of the lungs resulting in inhibitory effects such as apnea (H.M. Coleridge, 1986).

PSRs detect volume and position of the lungs via lung inflation and deflation (Adrian, 1933; Bailey \& Fregosi, 2006; H.M. Coleridge, 1986; Richardson, Herbert, \& Mitchell, 1984). The majority of PSRs are located in the intrapulmonary airway in dog (Bartlett, Sant'ambrogio, \& Wise, 1976; Miserocchi, Mortola, \& Sant'ambrogio, 1973), cat (Ravi, 1986; Widdicombe, 1954) and guinea pig (Keller, Kohl, \& Koller, 1989) as well as a smaller percentage located in other areas including the: intra and extrathoracic 
trachea and extrapulmonary bronchii, which correspond to myelinated afferent nerve fibers that innervate airway smooth muscle (Schelegle \& Green, 2001).

Paintal (1973) described two types of slowly adapting PSRs, low threshold, which phasically fire throughout the cycle providing positive feedback to inspiratory drive, and high threshold, which only fire during critical levels of inflation signaling for the switch to deflation (H.M. Coleridge, 1986). Inflation and deflation of the lungs appear to be dependent on the threshold and adaptation of PSRs (Larrabee \& Knowlton, 1946). They provide afferent feedback that contributes to the Hering-Breuer inflation reflex, which prevents over inflation of the lungs, as well as coordination of laryngeal, tongue and hyoid muscles during airway obstruction (Bailey \& Fregosi, 2006). The deflation reflex due to deflation of the lungs by compression or restriction of the chest and abdomen results in the vagal reflex tachypnea consisting of a shortened inspiratory duration and expiratory duration seen across dogs (Culver \& Rahn, 1952; D'Angelo, Miserocchi, \& Agostoni, 1976; Hammouda \& Wilson, 1939), cats (Adrian, 1933; D'Angelo, Miserocchi, \& Agostoni, 1976), rabbits (D'Angelo, Miserocchi, \& Agostoni, 1976; Davies, Dixon, Callanan, Huszczuk, Widdicombe, \& Wise, 1978; Widdicombe \& Sellick, 1970) guinea pigs (Koller \& Ferrer, 1970) and man (H.M. Coleridge, 1986).

CPGs are a network of neurons that produce rhythmic and repetitive impulses that control muscle activity in the absence of afferent feedback (Delcomyn, 1980; Dick, Oku, Romaniuk, \& Cherniack, 1993b; Richter, Ballantyne, \& Remmers, 1986), and in the presence of afferent feedback adjust to changing conditions (Dutschmann, Mörschel, Rybak, \& Dick, 2009). Respiratory CPGs (rCPG) are modulated by vagal afferent feedback from PSRs (Bautista \& Dutschmann, 2014; Dutschmann, Bautista, Mörschel, \& 
Dick, 2014; Dutschmann, Mörschel, Rybak, \& Dick, 2009). Frequency of PSRs discharge has a direct relationship with lung volume (Adrian, 1933; Keller \& Loeser, 1929; Larrabee \& Knowlton, 1946; Partridge, 1939; Widdicombe, 1954), the higher the frequency of discharge the greater the inflation or lung volume. As PSRs discharge during inspiration, it triggers an inspiratory off switch causing a decrease in discharge rate throughout expiration (Bradley, 1977; H.M. Coleridge, 1986; Schelegle \& Green, 2001).

PSRs send afferent information to ' $p$ (pump) cells' in the ventolateral NTS, more specifically the dorsal respiratory group, which are responsible for tracking lung volume changes by mediating the Hering-Breuer reflex and inhibiting neurons receiving afferent information from rapidly adapting receptors (Berger, 1977; Kubin, Alheid, Zuperku, \& McCrimmon, 2006). The p cells project and inhibit inspiratory neurons in the lateral respiratory column (Ezure \& Tanaka, 2004; Kubin, Alheid, Zuperku, \& McCrimmon, 2006) resulting in the inspiratory off switch. Within the NTS NMDA receptors are the primary relay mechanism for Hering-Breuer reflex (Bonham, 1995; Miyazaki, Tanaka, \& Ezure, 1999; Wasserman, Sahibzada, Hernandez, \& Gillis, 2000). The ventrolateral NTS has reciprocal connectivity with the VRC and the pontine Kölliker Fuse (Dobbins \& Feldman, 1994; Ellenberger \& Feldman, 1990; Ezure, Tanaka, Saito, \& Otake, 2002; Hayashi, Coles, \& McCrimmon, 1996; Herbert, Moga, \& Saper, 1990), while the Kölliker Fuse and VRC share connectivity as well (Segers, Nuding, Dick, Shannon, Baekey, Solomon, Morris, \& Lindsey, 2008). The NTS and Kölliker Fuse form the neuroaxis for inspiratory off switch (Dutschmann, Mörschel, Reuter, Zhang, Gestreau, Stettner, \& Kron, 2008). This inspiratory off switch is controlled by the PSR afferent 
feedback and have phasic synaptic interaction of the Kölliker Fuse and rCPG (Dutschmann \& Dick, 2012).

\section{Spinally mediated}

Lung volume is regulated indirectly via proprioceptive receptors in the thoracic cavity (Campbell \& Howell, 1962; Lust, 2007). Changes in length (volume) and tension (pressure) within the thoracic cavity produces feedback on the muscle length-tension relationship (Campbell \& Howell, 1962; Zechman Jr \& Wiley, 2011). Intercostal muscle spindles act as a "follow up length servo" and automatically corrects the length change in muscle fibers for each respiratory movement to match central demand for volume change in spite of load variations (Corda, Eklund, \& Von, 1965). For example, an increase in muscle tension during tracheal occlusion results in an increase in firing rate of golgi tendon organs (Shannon, Shear, Mercak, Bolser, \& Lindsey, 1985). Primary function of the intercostal muscle is to maintain proper rib placement in coordination with diaphragm contraction (Jaiswal \& Davenport, 2016) and stability during movement (De Troyer, Kirkwood, \& Wilson, 2005; Feldman, 1986). Due to higher density of muscle spindles in the rostral rather than caudal intercostal muscles, there is a greater contribution to tidal volume from rostral ribcage in times of high inspiratory drive (D'Angelo, 1982). Intercostal muscles are strongly susceptible to proprioceptive feedback from their muscle spindles, golgi tendon organs and costovertebral joint receptors (D'Angelo, 1982; Jaiswal \& Davenport, 2016). Intercostal muscles are modulated on a breath by breath basis (Jaiswal \& Davenport, 2016) and feedback to medullary respiratory centers (Shannon, Shear, Mercak, Bolser, \& Lindsey, 1985). It is widely accepted that golgi tendon organs, not muscle spindles, have an inhibitory effect on medullary inspiratory neurons (Bolser, 
Lindsey, \& Shannon, 1983, 1984) which lead to a decrease in diaphragm, intercostal and laryngeal muscle activity (Shannon, Bolser, \& Lindsey, 1987). Due to few to none muscle spindles in the diaphragm it is believed control of the diaphragm is not proprioceptive driven (D'Angelo, 1982). Lack of muscle spindles in the diaphragm has been associated with reasoning as to why intercostal muscle activity doubles that of diaphragm activity under conditions of high drive (D'Angelo, 1982).

Spinally mediated proprioception of the thoracic cavity was first suggested by Ramos Garcia (1959), Campbell (1962), and Eccles, Sears and Shealy (1962). Sumi (1963a), however was the first to suggest spinal influence on swallow activity. Chest compression in conjunction with vagotomy has been used to assess spinal influence on breathing (Culver \& Rahn, 1952; Shannon, 1975; Shannon, 1979b). Mechanistically it has been suggested that increase in respiratory rate during chest compression in the absence of vagal feedback is due to signal failure of intercostal mechanoreceptors in extrafusal muscle fibers to shorten at the same times as intrafusal muscle fibers (Shannon, 1979b). Stimulation of intercostal group I and II afferent fibers influence upper airway activity (Remmers, 1973; Shannon, Bolser, \& Lindsey, 1987). Stimulation of group I afferent fibers augmented thyroarytenoid muscle activity (Remmers, 1973) and attenuated PCA muscle activity (Shannon, Bolser, \& Lindsey, 1987).

Influence of vagal feedback from PSRs has been studied to a much greater depth than spinal feedback in both control of breathing and swallow behavior. The research in the series of projects, presented in the preceding chapters, sought to further investigate vagal and spinal influences on breathing, swallow and the coordination of these two behaviors. In the next few chapters, we will propose theories and mechanisms in both 
human and animal explaining the influence and important for a balance of both vagal and spinal feedback in order to maintain proper coordination of breathing and swallow. 


\section{CHAPTER 2}

STRATEGIES FOR THE INTEGRATION OF COUGH AND SWALLOW TO MAINTAIN AIRWAY PROTECTION IN HUMANS

\section{Introduction}

Airway protection, the ability to remove and/or prevent foreign materials from entering the airway, is mediated by behaviors such as cough, swallow, and breathing (Bolser, Gestreau, Morris, Davenport, \& Pitts, 2013a; Pitts, Morris, Lindsey, Davenport, Poliacek, \& Bolser, 2012a; Pitts, Rose, Mortensen, Poliacek, Sapienza, Lindsey, Morris, Davenport, \& Bolser, 2013a). Abnormalities of cough (dystussia) and/or swallow (dysphagia) results in increased risk for aspiration and/or pneumonia (Bolser, Gestreau, Morris, Davenport, \& Pitts, 2013a; Hammond, Goldstein, Horner, Ying, Gray, GonzalezRothi, \& Bolser, 2009; Pitts, Morris, Lindsey, Davenport, Poliacek, \& Bolser, 2012a; Pitts, Rose, Mortensen, Poliacek, Sapienza, Lindsey, Morris, Davenport, \& Bolser, 2013c; Smith Hammond, Goldstein, Zajac, Gray, Davenport, \& Bolser, 2001).

Previous work has demonstrated cough efficacy as an important factor in determining aspiration risk in neuro -traumatic or -degenerative populations (Hammond, Goldstein, Horner, Ying, Gray, Gonzalez-Rothi, \& Bolser, 2009; Smith Hammond, Goldstein, Zajac, Gray, Davenport, \& Bolser, 2001). This was first described in stroke, with significant cough impairments in patients who aspirated (Smith Hammond, 
Goldstein, Zajac, Gray, Davenport, \& Bolser, 2001). Pitts et al. as well as Hegland et al. (2014; 2014; 2016) confirmed these results in patients with Parkinson's disease (PD); demonstrating that cough and swallow are related behaviors and cough function can detect swallow impairment.

Noting these behavioral relationships, Pitts et al. (2013c) investigated the coordination of cough and swallow in cats using a protocol to stimulate aspiration. The results of Pitts et al. (2013c) suggest aspiration produces a meta-behavior response, indicating both swallow and cough are highly coordinated and follow an order of operations. While this has been elucidated in cats, mechanisms of cough and swallow coordination in humans have yet to be examined.

This study was aimed to examine a protocol for the integration of cough and swallow in healthy adults. We hypothesized that during cough there would be a significant increase in submental muscle complex activation, accompanied by a decrease in swallow duration. Additionally, there would be a significant increase in cough-related oblique muscle activity after swallow, similar to previous observations in cats.

\section{Methods}

This protocol was approved by the University of Louisville Institutional Review Board (IRB\# 07.0272). Seven healthy males (26 \pm 6 years) with an average body mass index of $23 \pm 2$ participated. Participants had no known history of vascular or heart/pulmonary disease, hypertension, diabetes, renal disease, neurological disease or trauma. All participants had no history of smoking within the last year prior to the study. Surface electromyography (sEMG) electrodes (Motion Lab Systems, Inc., Baton Rouge, LA) were placed on clean-shaven areas of the submental complex (under the 
chin) (Cook, Dodds, Dantas, Kern, Massey, Shaker, \& Hogan, 1989; Shaker, Dodds, Dantas, Hogan, \& Arndorfer, 1990), and right oblique. A combination of a spirometer and elastic bands were used to measure airflow and lung volume (LV). A spirometer flow head (FE141, ADInstruments Dunedin, New Zealand) was attached to an oval shaped disposable mouth-piece $(3.5 \mathrm{~cm} \times 2.54 \mathrm{~cm})$ and nose-clip was used. Elastic bands (Pneumotrace II, UFI, Morro Bay, CA) were placed around the ribcage (RC) and abdomen $(\mathrm{AB})$ to allow for measurement of $\mathrm{LV}$.

All data was recorded using LabChart at $10 \mathrm{KHz}$. Files were imported into Spike 2 version 8 (Cambridge Electronic Design, United Kingdom), low pass filtered at $200 \mathrm{~Hz}$, rectified, and smoothed at $20 \mathrm{~ms}$.

\section{Experimental Protocol}

Participants were seated upright and a technician held the spirometer/mouthpiece in place. Participants reported no previous training, exposure to equipment, and/or procedures (including researcher demonstration). Participants were asked to "relax and breathe" into the spirometer for at least three eupneic breath cycles before starting each protocol. Three different protocols were used to assess cough, swallow, and the combination of both cough and swallow. Two trials for each protocol were performed, and the same investigator performed all trials across all participants:

A. Voluntary Cough: Participants were asked to, "cough like there is something stuck in your throat."

B. Swallow stimuli: Participants were asked to, "swallow whenever you feel like you need to," and then 3cc's of water was steadily infused into their mouth over 30 seconds. 
C. Combined stimuli: Participants were asked to, "cough like something is stuck in your throat and swallow whenever you feel like you need to;" and following 1 full cough epoch (defined below) 3cc's of water was infused into their mouth over 30 seconds.

\section{$\underline{\text { Analysis }}$}

Detection of a cough epoch was noted by an increase in airflow on the spirometer channel, activation of oblique and the decrease in $\mathrm{RC}$ and $\mathrm{AB}$ bands (as shown in Figure 2-1 and 2-2). Swallow was verified by an absence of airflow and activation of submental complex sEMG (Figure 2-2, -4 and -5).

Airflow was continuously monitored during each protocol, and the following measurements were made, as modified from Pitts et al. (2009) (see Figure 2-1):

1) Inspiratory Phase Duration (IPD): onset of inspiration (flow < 0 ) to onset of compression phase (flow $=0)($ A to $\mathrm{C})$

2) Inspiratory Phase Peak Flow (IPPF): peak negative flow (B)

3) Inspiratory Phase Rise Time (IPRT): onset of inspiration (flow < 0 ) to the peak negative flow (A-B)

3) Compression Phase Duration (CPD): period of zero flow prior to an expiratory effort (C to D) and (F to $\mathrm{G})$

4) Expiratory Phase Rise Time (EPRT): end of zero flow to peak positive flow (D to E)

5) Expiratory Phase Peak Airflow (EPPF): peak positive airflow (E) 
Participants also performed a forced vital capacity (FVC) maneuver to establish LV. Maximum and minimum measurements of both $\mathrm{RC}$ and $\mathrm{AB}$ elastic bands during the FVC maneuver (Figure 2-2) were used to establish LV for each participant based on the following equations:

$$
\begin{aligned}
& \text { 1) } R C_{\text {max }}=F V C_{\text {max }}+\left|F V C_{\text {min }}\right| \\
& \text { 2) } A B_{\text {max }}=F V C_{\text {max }}+\left|F V C_{\text {min }}\right| \\
& \text { 3) } L V_{\text {max }}=0.7(R C)+0.3(A B) \\
& \text { 4) } R C_{x}=X_{\text {max }}+\left|F V C_{\text {min }}\right| \\
& \text { 5) } A B_{x}=X_{\text {max }}+\left|F V C_{\text {min }}\right| \\
& \text { 6) } \left.L V_{x}=0.7\left(R C_{x}\right)+0.3\left(A B_{x}\right) 7\right) \% V C=\frac{L V_{x}}{L V_{\max }}
\end{aligned}
$$

In order to calculate the "maximum range" of both the $\mathrm{RC}$ and $\mathrm{AB}$, maximum and absolute value of the minimum were added together for both $\mathrm{RC}$ and $\mathrm{AB}$, respectively (see equation 1 and 2). Using the equation from Mead et al. (1967) LV was calculated with $70 \%$ from chest wall expansion and 30\% from abdominal. In order to incorporate these contributions into our values, the "maximum range" of $\mathrm{RC}$ and $\mathrm{AB}$ was multiplied by 0.7 and 0.3 , respectively, then added together to generate maximum $L V\left(L V_{\max }\right)$ (see equation 3). To produce the $\% \mathrm{VC}$ of a single swallow, the $\mathrm{LV}$ of the individual swallow or cough $\left(\mathrm{LV}_{\mathrm{x}}\right)$ was identified using equations $4-6$, then divided by $L V_{\max }$, producing $\% \mathrm{VC}$.

\section{Statistical Analysis}

All results are expressed as means \pm standard deviation (SD) and analyzed using SPSS software (IBM). To identify significant changes between EMG, airflow, and LV measurements, a one-way ANOVA was used. A Wilcoxon Signed Ranks test was used to 
assess the respiratory/cough phase changes. A Students paired t-test was used to compare the duration of the compression phase with swallows occurring in the presence and absence of the compression phase, and operating volumes of both cough and swallow during each protocol. A Pearson Product moment correlation was used to evaluate the relationship between submental duration, amplitude, and swallow apnea duration. A pvalue of less than 0.05 was considered significant.

Results

\section{$\underline{\text { Cough }}$}

Cough trials elicited an average of $4.4 \pm 1.3$ epochs (single inspiration followed by multiple expirations) with an average of $2.3 \pm 0.4$ expiratory efforts per epoch. There was a significant increase in the number of cough epochs, $\left(8.6 \pm 2.6 ; \mathrm{F}_{1,12}=14.61, p<\right.$ 0.01) during the combined stimuli protocol (Table 2-1). Cough airflow analysis revealed significantly shortened IPD $\left(\mathrm{F}_{1,12}=10.18, p<0.01\right)$ and IPRT $\left(\mathrm{F}_{1,12}=8.40, p<0.05\right)$; and an increase in CPD without swallow $\left(\mathrm{F}_{1,12}=11.30, p<0.01\right)$ during the combined stimuli protocol (Table 2-1).

There was no significant change in IPPF, EPRT, or EPPF (Figure 2-1 and Table 2-1), or right oblique (RO) sEMG (\% of maximum) amplitude or duration during combined stimuli and cough protocols.

\section{$\underline{\text { Swallow }}$}

Water infusion elicited an average of $7.1 \pm 1.4$ swallows, which was not significantly different than swallows observed during the combined stimuli protocol (7.6 $\left.\pm 1.7 ; F_{1,12}=0.27, p=0.61\right)$. There were no significant changes in swallow-related submental sEMG (\% of maximum) amplitude when comparing swallows during 
breathing to swallows in the combined stimuli protocol $\left(\mathrm{F}_{1,12}=0.14, p=0.72\right)$, as well as no significant change in swallow related apnea duration (swallow stimuli: $1.1 \pm 0.3 \mathrm{~s}$, combined stimuli: $\left.1.2 \pm 0.2 ; \mathrm{F}_{1,12}=0.02, p=0.88\right)($ Table $2-1)$.

There was a significant increase in compression phase duration with swallows occurring during the compression phase $(1.4 \pm 0.3 \mathrm{~s})$ compared to swallows not occurring in the compression phase $\left(1.1 \pm 0.5 \mathrm{~s} \mathrm{t}_{5}=4.07, p<0.01\right)$.

Pearson Product correlations resulted in moderate-positive relationships between submental duration and submental amplitude $(r=0.4, p<0.01)$, and submental duration and swallow apnea duration $(r=0.55, p<0.01)$. A weak-positive relationship was shown between submental amplitude and swallow apnea duration $(r=0.20, p<0.05)$.

\section{Cough and respiratory phase}

During tidal breathing $56 \%$ percent of swallows (30 of 54) occurred during the transition from inspiration to expiration (In-Ex); 37\% (20 of 54) occurred during expiration (Ex-Ex); and 7\% (4 of 54) occurred during the inspiratory phase (In-In). During the combined stimuli protocol $46 \%$ of swallows (25 of 55) occurred during a cough inspiration (cIn-cIn); $42 \%$ occurred during a compression phase [i.e. transition from inspiration to expiration (cIn-cEx, 18 of 55) or expiration to expiration (cEx-cEx, 5 of 55)]; and only $13 \%$ occurred (7 of 55) during the transition of the cough expiration and cough inspiration (cEx-cIn) (Figure 2-2 and -3). A Wilcoxon signed ranks test indicated a change in phase preference from breathing to cough $[\mathrm{Z}=-5.378, p<0.001]$, with the InEx pattern preferred during eupnea and cIn-cIn pattern preferred during the combined stimuli protocol. 


\section{$\underline{\text { Lung volume }}$}

Swallow tended to occur in a narrow range of lung volume. During swallow stimuli trials, average lung volume was $44 \pm 7 \% \mathrm{VC}$, and $48 \pm 8 \% \mathrm{VC}$ during combined stimuli trials $\left(\mathrm{F}_{1,12}=0.84, p=0.38\right)$ (Figure 2-3). During cough trials, the lung volume ranged between $48.2 \% \mathrm{VC}$ and $97.5 \%$ of VC. During the combined stimuli protocol, average maximum lung volume was $89.2 \%$ of $\mathrm{VC}$, while the average minimum lung volume was $49.7 \%$ of VC. Despite the minor reductions in range of lung volumes, there were no change in \% VC between the two protocols when looking at cough expiration (21 $\pm 11 \%, 23 \pm 5 \%)$ and cough inspiration $(71 \pm 15 \%, 66 \pm 13 \%)$ in the cough and combined stimuli protocols, respectively (Table 2-1).

\section{Discussion}

While cough and swallow have been independently studied in many patient populations e.g. (Hegland, Davenport, Brandimore, Singletary, \& Troche, 2016; Hegland, Okun, \& Troche, 2014; Pitts, Bolser, Rosenbek, Troche, \& Sapienza, 2008; Smith Hammond, Goldstein, Zajac, Gray, Davenport, \& Bolser, 2001), this is the first study to examine the coordination of cough and swallow in humans. The current study had several major findings: 1) cough and swallow can be reliably integrated; 2) the combined stimuli protocol resulted in more cough epochs and a decrease in the IPD and CPD; and 3) unexpectedly, participants were willing to swallow during any cough phase, using multiple strategies to maintain LV.

\section{Lung volume and phase preference}

Swallowing during eupnea has been intensely studied. Two primary features of these studies are the phase preference for swallows to occur during the expiratory phase 
of breathing (Martin-Harris, Brodsky, Price, Michel, \& Walters, 2003a; Wheeler Hegland, Huber, Pitts, Davenport, \& Sapienza, 2011b; Wheeler Hegland, Huber, Pitts, \& Sapienza, 2009b) and optimal LV targets ( 44\%VC) (McFarland, Martin-Harris, Fortin, Humphries, Hill, \& Armeson, 2016; Wheeler Hegland, Huber, Pitts, Davenport, \& Sapienza, 2011b). Our observations are indicative of a more rigid regulatory control system of swallow. We propose the concept of volume targeting to explain the fixed occurrence of swallow in a small LV range during eupnea and repetitive cough. This idea is reinforced by our stable LV during swallow across the two protocols. Additionally, Figure 2-2, which demonstrates the four primary strategies for cough-swallow coordination including swallowing during the expiratory phase of a cough epoch (B) and most strikingly, swallowing during the inspiratory phase of cough (D and Figure 2-4).

Volume related feedback is mainly accomplished by pulmonary stretch receptors (PSR) activation (Canning, Mori, \& Mazzone, 2006; Clark \& von Euler, 1972; Poliacek, Simera, Veternik, Kotmanova, Pitts, Hanacek, Plevkova, Machac, Visnovcova, Misek, \& Jakus, 2016). PSR feedback functions as an "inspiratory off switch" when a target LV/threshold has been achieved (Adrian, 1933; Bradley, von Euler, Marttila, \& Roos, 1975; Clark \& von Euler, 1972; E \& Agostoni, 1975; H.M. Coleridge, 1986; Karczewski, 1962; Trenchard, 1977; Widdicombe, 1964), and we have many examples of coughrelated inspiration being interrupted at a "target" LV for the execution of swallow (Figure 2-4). Work in cats and rabbits have demonstrated: a) PSRs have a significant influence on cough reflex (Poliacek, Simera, Veternik, Kotmanova, Pitts, Hanacek, Plevkova, Machac, Visnovcova, Misek, \& Jakus, 2016), and b) when chemically blocked there is a decrease in cough frequency and intensity (Bucher, 1958; Hanacek, Davies, \& Widdicombe, 1984; 
Sant'Ambrogio, Sant'Ambrogio, \& Davies, 1984). Pitts, et al (2013a) hypothesized that coordination of cough and swallow was mainly accomplished through phase specific information (which does encompasses LV). However, in humans, volume specific information may be of greater consequence.

During tidal breathing, respiratory-swallow coordination patterns can occur at four points in the respiratory cycle: 1) In-Ex, 2) Ex-Ex, 3) Ex-In, or 4) In-In (Wheeler Hegland, Huber, Pitts, \& Sapienza, 2009b). With the use of oral spirometry and elastic bands we found $56 \%$ of swallows occurred during In-Ex phase and only $37 \%$ of swallows during Ex-Ex phase in the swallow stimuli protocol. In contrast, previous studies that used nasal airflow recording and elastic bands found that $73 \%$ to $79 \%$ of swallows during the Ex-Ex phase pattern (Brodsky, McFarland, Dozier, Blair, Ayers, Michel, Gillespie, Day, \& Martin-Harris, 2010; Wheeler Hegland, Huber, Pitts, \& Sapienza, 2009b). We believe the findings of this study differed from other studies with a similar swallow protocol due at least in part to the spirometer mouthpiece. There have been studies that evaluate the effects of different respiratory apparatuses on gas exchange and breathing patterns (Askanazi, Silverberg, Foster, Hyman, Milic-Emili, \& Kinney, 1980; Wohlgemuth, van der Kooi, Hendriks, Padberg, \& Folgering, 2003), but there are no studies to our knowledge that clarify the impact of an "open mouth" on phase/timing of swallow. We also acknowledge the oral mouthpiece is a limitation to this current study, however it was specifically chosen for comparison to all recently published cough work. In the future this study should be replicated using either a facemask or without spirometry, to allow for more "natural" swallowing. 


\section{$\underline{\text { Swallows during the compression phase of cough }}$}

Unexpectedly, swallow occurred at any time during a cough, including the compression phase of cough. In these instances, swallow either occurred immediately post inspiratory phase just before the first expiratory effort (33\% of cough-swallows, Figure 2-5A) or swallow occurred in between two expiratory efforts with a prolonged apnea interrupting the cough epoch (9\% of cough-swallows, Figure 2-5B). In both types of compression phase swallows, expiratory abdominal muscles remained inactive until the swallow was completed then immediately activated, and the CPD increased. This increase may be due to the need of abdominal muscle activation in preparation for effective shearing forces during the cough expiration (Figure 2-5). The phenomenon of swallow suppressing active abdominal recruitment has also been demonstrated in expiratory threshold loading (Pitts, Gayagoy, Rose, Poliacek, Condrey, Musslewhite, Shen, Davenport, \& Bolser, 2015b).

\section{$\underline{\text { Apnea Duration }}$}

Swallow related apnea has been studied across many different populations, genders, ages and conditions all in healthy adult humans, but this is the first study, to our knowledge, to study apnea duration in coordination with cough. Studies report swallow related apnea durations ranging from 0.93-1.5s (Clark, 1920; Hiss, Treole, \& Stuart, 2001; Kijima, Isono, \& Nishino, 1999; Martin, Logemann, Shaker, \& Dodds, 1994b; Nishino, Yonezawa, \& Honda, 1985; Yagi, Oku, Nagami, Yamagata, Kayashita, Ishikawa, Domen, \& Takahashi, 2017) in healthy adult human subjects. Swallow apnea duration was determined at normocapnic and hypercapnic conditions 1.3 and $0.8 \mathrm{~s}$, respectively by Hardemark et al. (2009). In our study we show swallow related apnea 
duration has no change when comparing between the swallow stimuli and combined stimuli trials. We have found in our study the apnea duration during swallow to be $1.1 \mathrm{~s}$ and 1.2s for swallow stimuli and combined stimuli trials, respectively, and within the range of previous reports (Clark, 1920; Hiss, Treole, \& Stuart, 2001; Kijima, Isono, \& Nishino, 1999; Martin, Logemann, Shaker, \& Dodds, 1994b; Nishino, Yonezawa, \& Honda, 1985; Yagi, Oku, Nagami, Yamagata, Kayashita, Ishikawa, Domen, \& Takahashi, 2017).

\section{$\underline{\text { Instructions }}$}

Instructions for voluntary cough behaviors have changed since Smith-Hammond evaluated their subjects by instructing, "breathe quietly for $30 \mathrm{~s}$ " and then repeatedly requested to, "voluntarily produce a strong cough" for three trials. Pitts et al $(2009 ; 2008$; 2010) studies used the set of instructions, "take a deep breath and cough hard" to conduct voluntary cough and asks the participant to continuously swallow the administered three ounces of thin liquid (Pitts, Troche, Mann, Rosenbek, Okun, \& Sapienza, 2010). Martin Harris used, "after I remove the syringe from your lip you may swallow whenever you feel comfortable," (Martin, Logemann, Shaker, \& Dodds, 1994b) and in later studies the instructions read, "drink the liquid in your usual manner" (Brodsky, McFarland, Dozier, Blair, Ayers, Michel, Gillespie, Day, \& Martin-Harris, 2010). Hegland and Troche more recently have used, "cough like something went down the wrong pipe" (Brandimore, Troche, Huber, \& Hegland, 2015). As have been reported by others, an instruction of, "cough like there is something in your throat" produces a robust and reliable response (Brandimore, Troche, Huber, \& Hegland, 2015). Common for cued swallow is, have the participant hold the bolus in their mouth and swallow when prompted/ready (Smith 
Hammond, Goldstein, Horner, Ying, Gray, Gonzalez-Rothi, \& Bolser, 2009), however McFarland, (2016) demonstrated that cueing can have a significant effect on LV during swallowing.

During this study we took special care to not give any behavioral demonstrations (i.e. experimenter producing an example cough bout), or answer any participant questions about the optimal behavioral output (they were only reminded of the original instruction). The repetitive cough challenge offered a unique experimental condition in which very few instructions were given to the participant, but they moved through a wide range of LV. This is probably the primary reason for the variety of strategies the participants used to complete the task, and offers a unique physiologic perspective. We fear the historic use of cues decreases natural behavioral variability and limits early diagnoses of "subtle" airway protective changes.

\section{Conclusion}

In conclusion, we have developed a novel protocol to test the aspiration response in the laboratory; and produces a range of behavioral responses that could be used to detect early pathologic changes in airway protection. Our results highlight the use of targeted lung volume for initiation of swallow instead of cough phase. This novel method could help clinicians train and/or develop therapies tailored to humans. 


\section{${ }^{1}$ Table 2-1.}

Means, standard deviations (SD), and p-value comparing cough protocol to combined $\underline{\text { stimuli protocol as well as swallow stimuli protocol to combined stimuli protocol for all }}$ measurements made in this study.

\begin{tabular}{|c|c|c|c|c|c|c|c|c|c|}
\hline & \multicolumn{4}{|c|}{ Single Stimuli } & \multicolumn{4}{|c|}{ Combined Stimuli } & \multirow[b]{2}{*}{$p$-value } \\
\hline & mean & ( & SD & ) & mean & ( & SD & ) & \\
\hline \multicolumn{10}{|l|}{ Cough Measures } \\
\hline $\operatorname{IPPF}(\mathrm{L} / \mathrm{s})$ & -3.6 & ( & 1.8 & ) & -3.3 & ( & 1.2 & ) & 0.72 \\
\hline IPRT (ms) & 889 & ( & 296 & ) & 514 & ( & 171 & ) & 0.013 \\
\hline IPD (ms) & 1244 & ( & 248 & ) & 882 & ( & 169 & ) & 0.008 \\
\hline CPD (ms) & 201 & ( & 31 & ) & 309 & ( & 80 & ) & 0.006 \\
\hline EPRT (ms) & 64 & ( & 69 & ) & 68 & ( & 69 & ) & 0.92 \\
\hline EPPF (L/s) & 8.9 & ( & 1.7 & ) & 8.2 & ( & 1.5 & ) & 0.43 \\
\hline Epochs & 4.4 & ( & 1.3 & ) & 8.6 & ( & 2.6 & ) & 0.002 \\
\hline EE/Epoch & 2.3 & ( & 0.4 & ) & 2.3 & ( & 0.6 & ) & 0.98 \\
\hline Inspiratory $\% \max \mathrm{VC}$ & 71 & ( & 15 & ) & 66 & ( & 13 & ) & 0.48 \\
\hline Expiratory $\% \max \mathrm{VC}$ & 21 & ( & 11 & ) & 23 & ( & 5 & ) & 0.67 \\
\hline Abdominal max EMG & 66 & ( & 11 & ) & 57 & ( & 10 & ) & 0.14 \\
\hline Abdominal EMG duration (ms) & 285 & ( & 103 & ) & 257 & ( & 80 & ) & 0.59 \\
\hline \multicolumn{10}{|l|}{ Swallow Measures } \\
\hline Swallow Frequency & 7.1 & ( & 1.4 & ) & 7.6 & ( & 1.7 & ) & 0.61 \\
\hline Apnea duration (ms) & 1136 & ( & 264 & ) & 1153 & ( & 160 & ) & 0.88 \\
\hline Swallow \% VC & 44 & ( & 7 & ) & 48 & ( & 8 & ) & 0.38 \\
\hline Submental \% max EMG & 66 & ( & 10 & ) & 68 & ( & 10 & ) & 0.72 \\
\hline Submental EMG duration(ms) & 1067 & ( & 520 & ) & 1108 & ( & 549 & ) & 0.89 \\
\hline
\end{tabular}

${ }^{1}$ Reprinted with permission from Huff, Reed, Smith, Brown, Ovechkin, and Pitts, 2018 Strageties for the integration of cough and swallow to maintain airway protection in humans Lung, 2018: p. 1-8. 
Inspiratory phase peak flow (IPPF), inspiratory phase rise time (IPRT), inspiratory phase duration (IPD), compression phase duration (CPD) without swallow, expiratory phase rise time (EPRT), expiratory phase peak flow (EPPF). Epochs is an inspiration followed by multiple expiratory efforts, (see Figure 2-2). The number of expiratory efforts per epoch (EE/Epoch). Inspiratory \% max VC is also known as operating volume for cough and swallow \% VC is the operating volume for swallow. 
${ }^{1}$ Figure 2-1. Cough epochs can be characterized using airflow and oblique surface EMG activity.

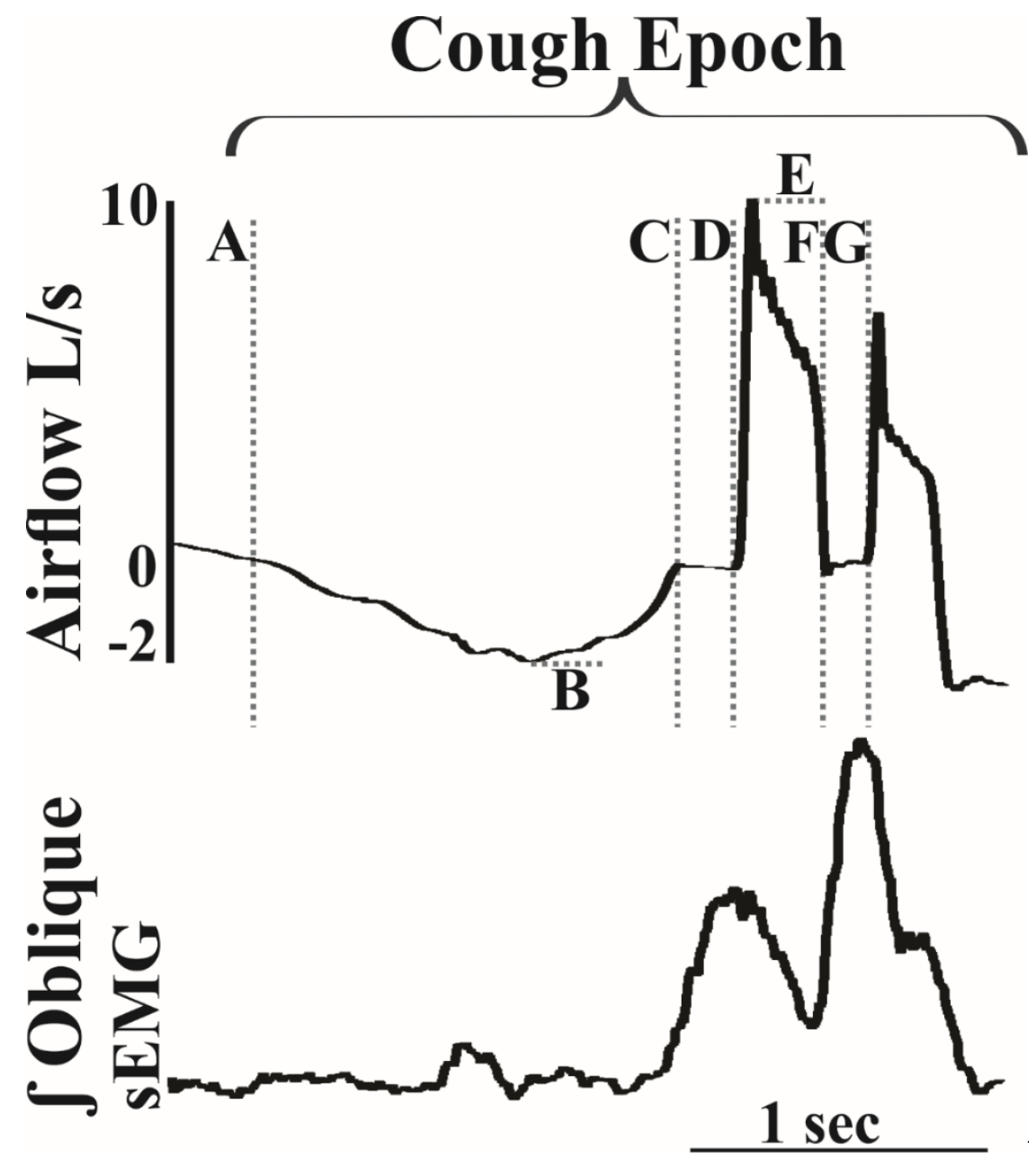

Airflow and integrated sEMG traces $(\oint)$ from the oblique muscle were measured during cough epochs (inspiratory phase followed by multiple expiratory efforts). The dotted lines represent phases and components of each cough epoch measured: Inspiratory phase duration (IPD) (A to C); inspiratory phase peak flow (IPPF) (B); inspiratory phase rise time (IPRT) (A-B); compression phase duration (CPD) (C to D) particularity an 
inspiratory to expiration compression phase; expiratory phase rise time (EPRT) (D to E); expiratory phase peak flow (EPPF) (E); and expiratory to expiratory CPD (F to G). 
${ }^{1}$ Figure 2-2. Examples of swallow occurring during each phase of cough.

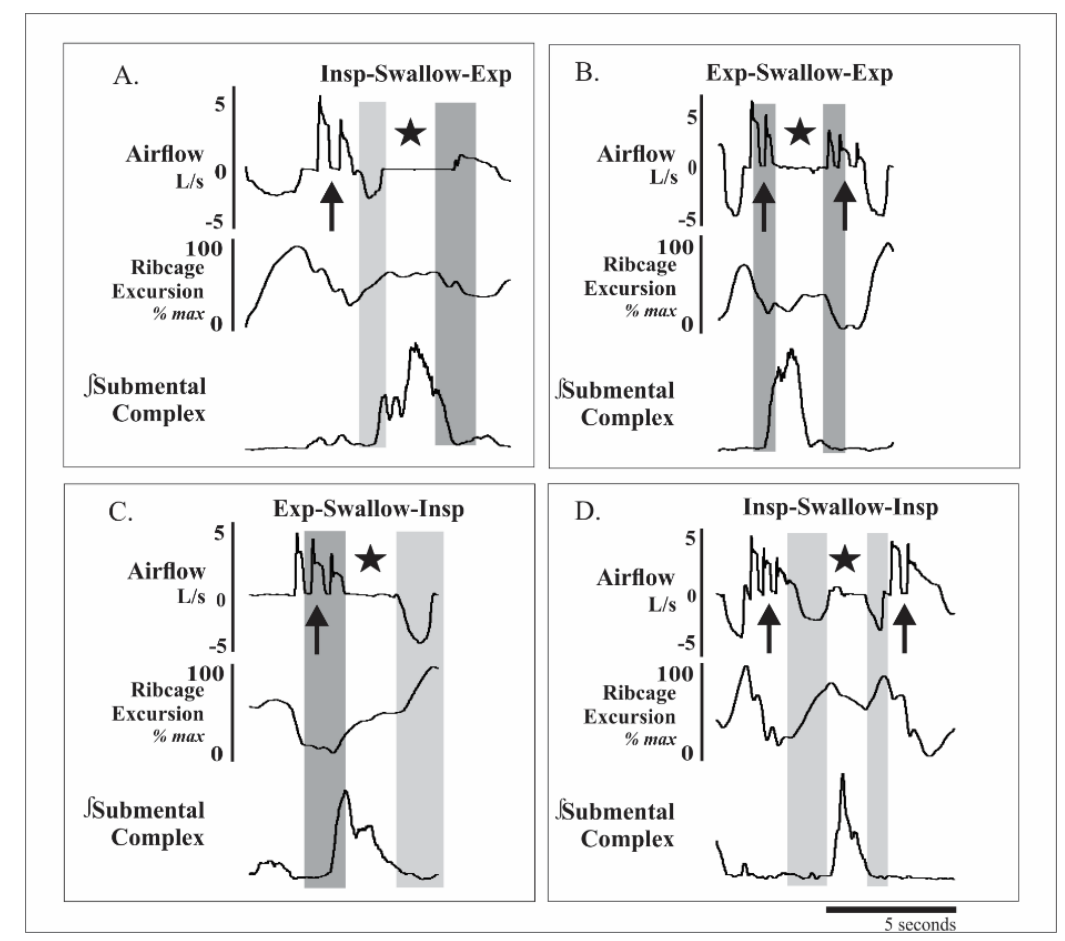

(A) Swallow can occur during the transition from the inspiratory phase (light gray rectangle) to the expiratory phase (dark grey rectangles; I-E), (B) in the middle of the expiratory phase (E-E), (C) during the transition from expiration to inspiration (E-I), or (D) in the middle of the inspiratory phase (I-I). Swallow activity ( $\star$ ) is confirmed by coincident activation of the submental complex and absence of airflow. Cough activity

${ }^{1}$ Reprinted with permission from Huff, Reed, Smith, Brown, Ovechkin, and Pitts, 2018 Strageties for the integration of cough and swallow to maintain airway protection in humans Lung, 2018: p. 1-8. (Figure 2) 
(†) is confirmed by sharp increases in airflow. Ribcage excursion demonstrates the ability of swallow to interrupt phases of breathing and coughing. 
${ }^{1}$ Figure 2-3. Swallows occurring during the inspiratory phase of cough.
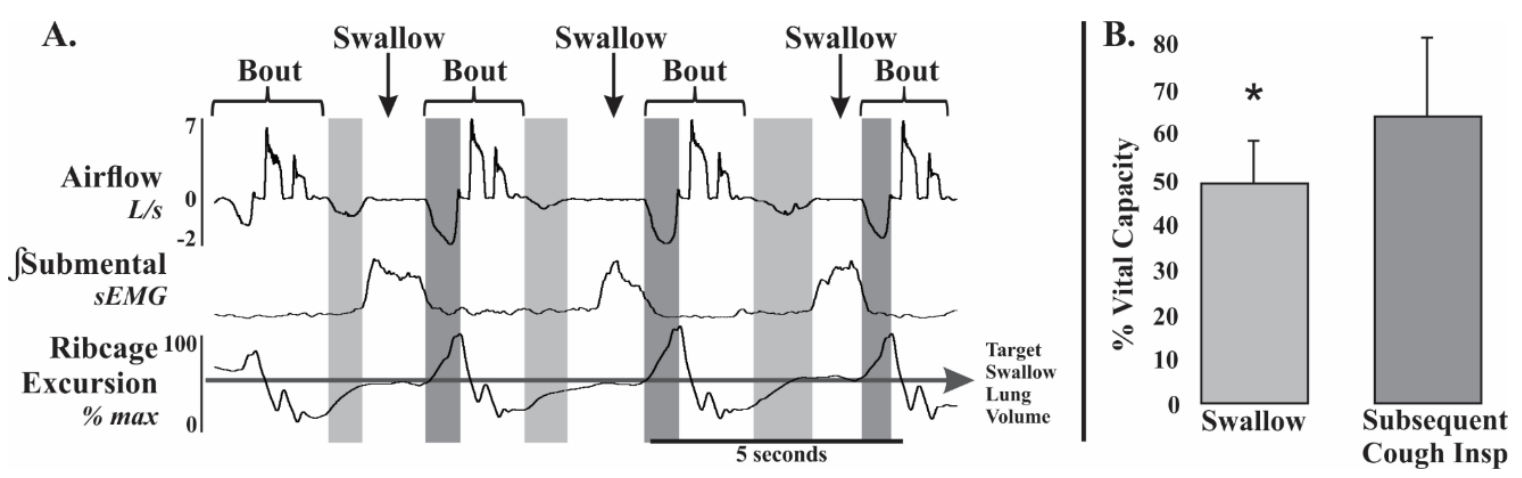

(A) demonstrates a consistent lung volume, \% vital capacity (VC), throughout the combined stimuli protocol. The LV required to initiate swallow (light grey boxes in both A and B) compared to the $\mathrm{LV}$ required to initiate an efficient cough (dark grey boxes in both $\mathrm{A}$ and $\mathrm{B}$ ) is significantly different (B). Average pre swallow inspiration $\% \mathrm{VC}$ is 48 \pm 8 and average pre cough inspiration $\% \mathrm{VC} 66 \pm 13, * p<0.01$. Shown are the intergral sEMG traces $\left(\int\right)$ filtered, smoothed and rectified. Each airflow and EMG shown are scaled to the same degree.

\footnotetext{
${ }^{1}$ Reprinted with permission from Huff, Reed, Smith, Brown, Ovechkin, and Pitts, 2018 Strageties for the integration of cough and swallow to maintain airway protection in humans Lung, 2018: p. 1-8. (Figure 3)
} 
${ }^{1}$ Figure 2-4. Abdominal EMG depression during compression phases which include swallow.

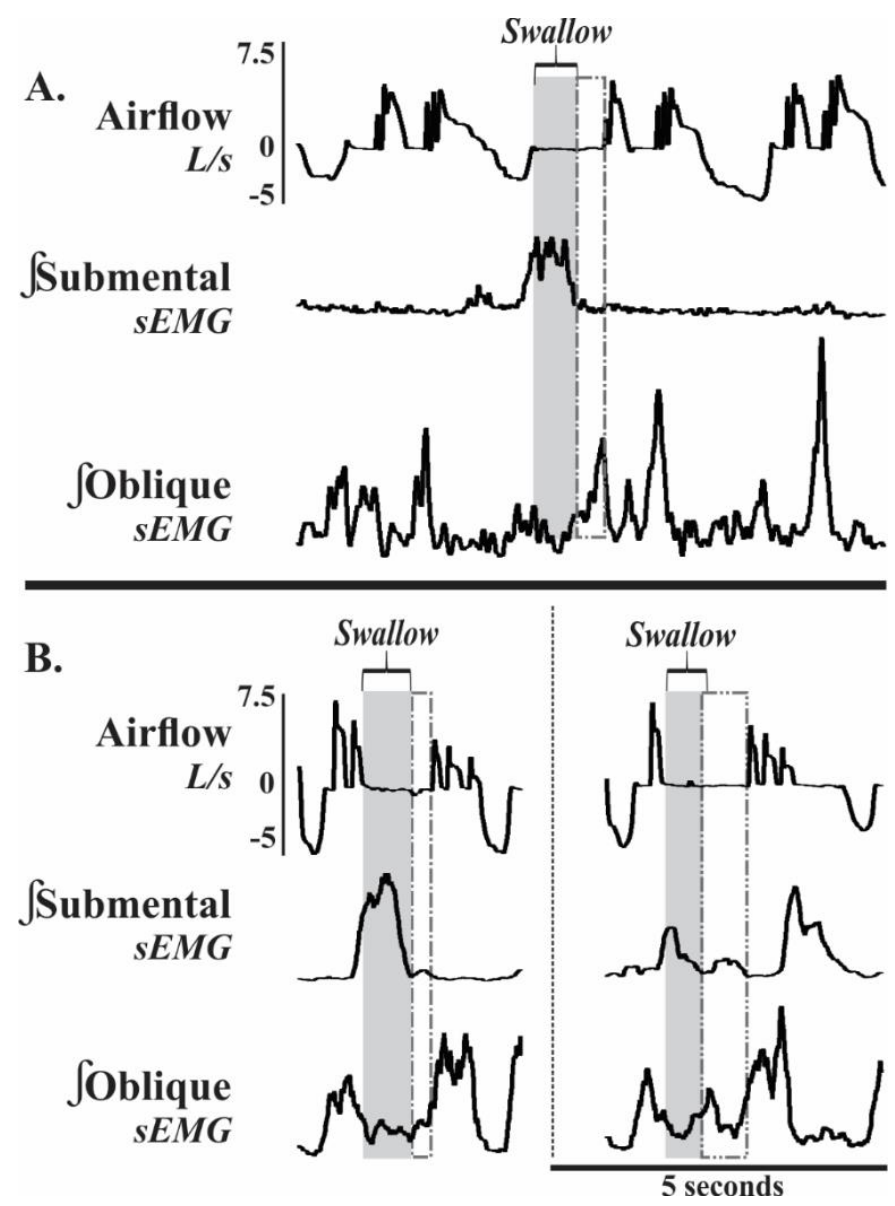

(A) Integrated submental and abdominal sEMG traces $(\delta)$ and airflow measurements show swallow (gray rectangels) and the activation of oblique muscle complex (dashed rectangles), extending the duration of the compression phase. ( $\mathrm{B}$, both panels). Of note,

${ }^{1}$ Reprinted with permission from Huff, Reed, Smith, Brown, Ovechkin, and Pitts, 2018 Strageties for the integration of cough and swallow to maintain airway protection in humans Lung, 2018: p. 1-8. (Figure 4) 
this extended compression phase may be due to the need of abdominal muscle activation in preparation for effective shearing forces during the cough expiration. 


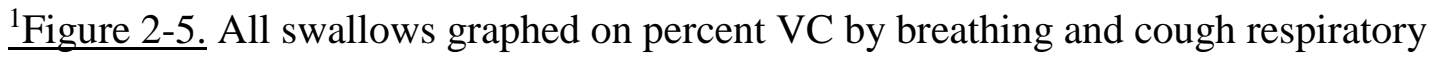
phase.
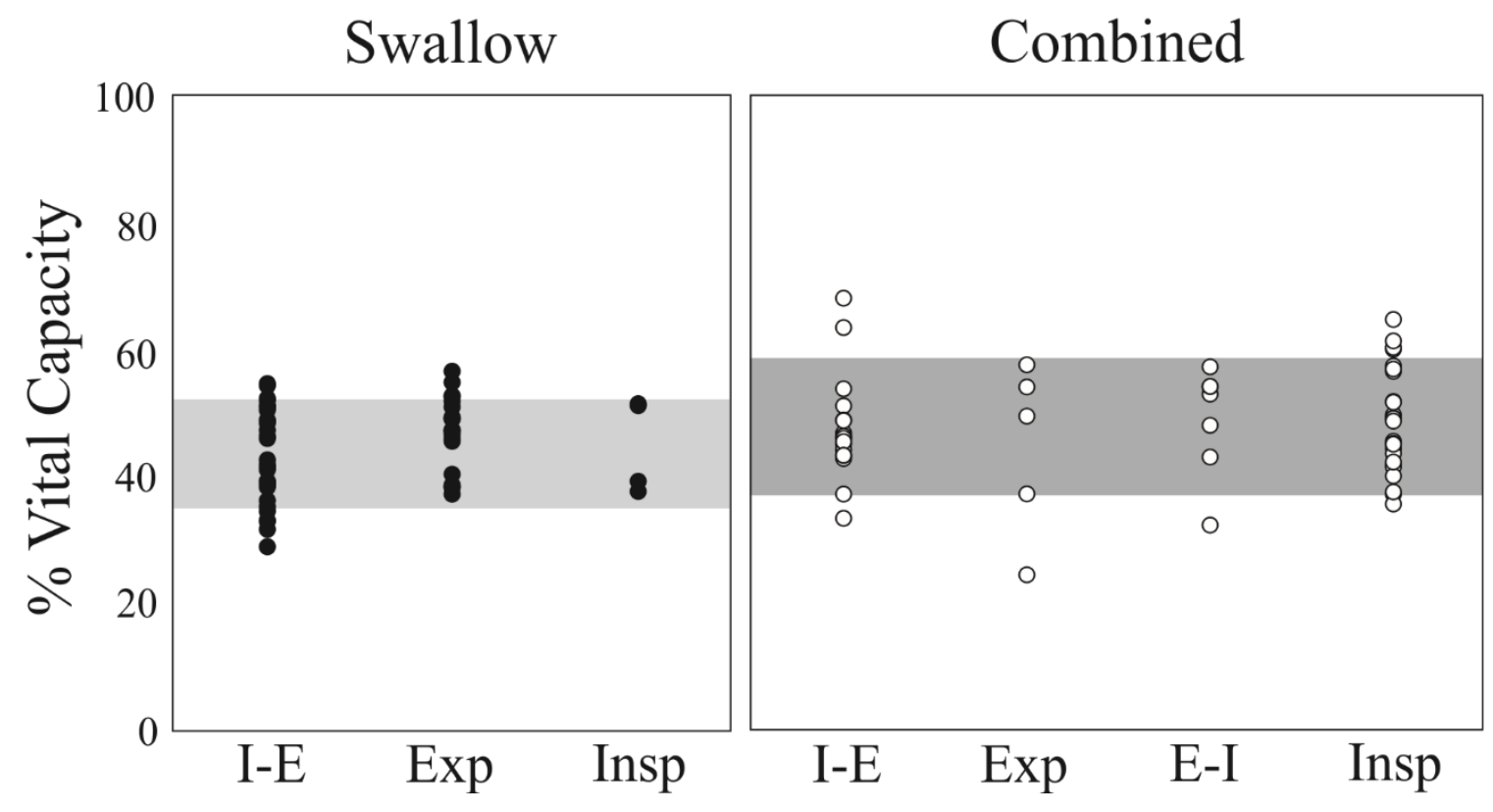

Swallows during breathing (left panel; filled circles) primarily occurred during the transition from inspiration to expiration (I-E) and in expiration (E). There were no occurrences of swallows occurring during the transition from expiratory to inspiratory phase (E-I; not shown) in the swallow stimuli protocol. Regardless of the phase of respiration, $90 \%$ of swallows (horizontal box) were observed between $35-52 \%$ VC. In the combined stimuli protocol (right panel; open circles) majority of swallows occurred during the inspiratory phase (I) or the transition from inspiration to expiration (I-E).

${ }^{1}$ Reprinted with permission from Huff, Reed, Smith, Brown, Ovechkin, and Pitts, 2018 Strageties for the integration of cough and swallow to maintain airway protection in humans Lung, 2018: p. 1-8. (Figure 5) 
Regardless of cough and breathing coordination, $90 \%$ (horizontal box) of swallows occur between $37-59 \%$ VC. 


\section{CHAPTER 3}

\section{SWALLOW-BREATHING COORDINATION DURING INCREMENTAL ASCENT}

\section{TO ALTITUDE}

\section{Introduction}

Swallow $^{2}$ and breathing are highly coordinated airway protective behaviors. Swallow is a multi-phase event, however the pharyngeal phase presents the highest risk for aspiration (Paydarfar, Gilbert, Poppel, \& Nassab, 1995). During the pharyngeal phase, supra-laryngeal/hyoid musculature moves the larynx superiorly and anteriorly resulting in closure of the airway and a functional apnea (German, Crompton, \& Thexton, 2009; Wheeler Hegland, Huber, Pitts, Davenport, \& Sapienza, 2011a; Wheeler Hegland, Huber, Pitts, \& Sapienza, 2009a).

The expiratory phase of breathing is the preferred phase for swallow to occur, likely due to the limited inspiratory airflow (Martin-Harris, Brodsky, Price, Michel, \& Walters, 2003b). The central mechanism is thought to be due to interactions of breathing and swallow pattern generators (Dick, Oku, Romaniuk, \& Cherniack, 1993a; Miller, 1982b), however this preference can be modified by peripheral feedback (Pitts, Rose, Poliacek,

\footnotetext{
${ }^{2}$ Reprinted with permsisson from Huff, Day, English, Reed, Zouboules, Saran, Leacy, Mann, Peltonen, O'Halloran, Sherpa and Pitts. Swallow-breathing coordination during incremental ascent to altitude. Respiratory Physiology \& Neurobiology, 2018.
} 
Condrey, Davenport, \& Bolser, 2015) and disease (Brodsky, McFarland, Dozier, Blair, Ayers, Michel, Gillespie, Day, \& Martin-Harris, 2010; Leslie, Drinnan, Ford, \& Wilson, 2002; Troche, Huebner, Rosenbek, Okun, \& Sapienza, 2011). Specifically, alterations in respiratory mechanics due to chronic obstructive pulmonary disease (Nagami, Oku, Yagi, Sato, Uozumi, Morita, Yamagata, Kayashita, Tanimura, Sato, Takahashi, \& Muro, 2017; Pinto, Balasubramanium, \& Acharya, 2017) and/or upper abdominal laparotomy can shift swallow occurrences to inspiration, potentially increasing risk of aspiration (Pitts, Rose, Poliacek, Condrey, Davenport, \& Bolser, 2015). Additionally, there is also limited evidence that alterations in blood gasses (i.e., oxygen $\left[\mathrm{O}_{2}\right]$ and carbon dioxide $\left[\mathrm{CO}_{2}\right]$ ) can also increase the likelihood that swallow will occur during inspiration (D'Angelo, DiazGil, Nunn, Simons, Gianatasio, Mueller, Meyer, Pierce, Rosow, \& Eikermann, 2014), (Ghannouchi, Duclos, Marie, \& Verin, 2013).

Incremental ascent to high altitudes $(>2,000 \mathrm{~m})$ produces hypoxia $\left(\right.$ low $\left.\mathrm{O}_{2}\right)$ induced hyperventilation, resulting in hypocapnia (low $\mathrm{CO}_{2}$ ) (Huang, Alexander, Grover, Maher, McCullough, McCullough, Moore, Sampson, Weil, \& Reeves, 1984; Weil, 1986). As climbers acclimatize to high altitude they can reach a new "steady-state chemoreflex drive" in which balance is achieved between hypoxia and hypocapnia, while ventilation parameters can return to near baseline conditions (Bruce CD, 2018; Pfoh, Steinback, Berg, Bruce, \& Day, 2017). Additionally, healthy individuals that are not acclimatized to high altitude conditions can have changes in pulmonary mechanics due to interstitial pulmonary edema, which can be accompanied with accumulation of fluid within and around the airway walls (Cremona, Asnaghi, Baderna, Brunetto, Brutsaert, Cavallaro, Clark, Cogo, Donis, \& Lanfranchi, 2002; Pratali, Cavana, Sicari, \& Picano, 2010; 
Schoene, Swenson, Pizzo, Hackett, Roach, Mills Jr, Henderson Jr, \& Martin, 1988).

Early symptoms such as shortness of breath and cough are often overlooked leading to mortality (Dunin-Bell \& Boyle, 2009).

Due to the significant coordination necessary for swallow and breathing, it is likely that conditions which significantly alter respiratory drive and mechanics would also affect swallow production and swallow-breathing coordination. We hypothesized that with incremental ascent to high altitude there would be a decrease in swallow duration, and a shift in swallow phase preference to inspiration.

\section{Methods}

\section{Ethics and Participant Recruitment}

This study abided by the Canadian Government Tri-Council policy on research ethics with human participants (TCPS2) and the Declaration of Helsinki, except for registration in a database. Ethical approval was received in advance through Mount Royal University Human Research Ethics Board (Protocol 100012) and was harmonized with the Nepal Health Research Council (Protocol 109-2017). Participants were recruited via email correspondence or direct verbal communication, and provided written, voluntary, informed and ongoing consent.

Ten participants were recruited for the study, while only seven (two males, five females) completed the study. One participant voluntarily withdrew from the study during ascent, another was excluded following baseline data acquisition due to a persistent cough and a third was excluded due to complications with data acquisition. Exclusion criteria included facial hair, as electrodes were unable to effectively adhere to skin, and health status (e.g., persistent cough, severe altitude illness). No pre-existing 
medical conditions were reported by any participants. Participants avoided rigorous exercise for at least 12 hours prior to data collection.

\section{Incremental ascent to high altitude}

Baseline measurements were recorded at 1,045m (Calgary) prior to the departure to Nepal. Following arrival in Kathmandu $(1,400 \mathrm{~m})$, participants spent up to 3 days in Kathmandu before flying to Lukla $(2,860 \mathrm{~m})$ where the trek to high altitude commenced (Figure 3-1). Consecutive measurements were obtained on rest days at 3,440m (Namche; day 3 at altitude) and 4,371m (Pheriche; day 5 at altitude) on every second day following arrival in Lukla (Figure 3-1), following one night sleep at each respective altitude.

\section{Data Collection}

Data acquisition was performed using an analog to digital data acquisition system [Powerlab/16SP ML880; AD Instruments (ADI), Colorado Springs, CO, USA], and data was collected, archived and analyzed offline using commercially available software (LabChart Pro software version 8) and a personal laptop computer. Surface electromyogram (sEMG) (ADI MLA2503 \& ADI FE132) electrodes were placed approximately $3 \mathrm{~cm}$ posterior to the mental region of the mandible, on each side of the midline, capturing the submental complex. The grounding electrode was placed inferior to the participant's left clavicle. Voluntary swallow was performed in advance to ensure an adequate electrical signal through the sEMG electrodes.

A pneumotachometer (800L flow head; Series 3813; Hans Rudolph Inc.) and spirometer amplifier (ADI ML141) were used to monitor respiratory variables using a mouthpiece and nose-clip. Calibration of the flow head was performed with a 3L calibration syringe before data acquisition in each participant. Respiratory flow (L/s) was 
measured directly by the pneumotachograph. Inspired volume $\left(\mathrm{V}_{\mathrm{TI}} ; \mathrm{L}\right)$ and respiratory frequency $\left(f_{\mathrm{R}} ; \min ^{-1}\right)$ were derived from respiratory flow. The product of $\mathrm{V}_{\mathrm{TI}}$ and $f_{\mathrm{R}}$ was used to determine instantaneous minute ventilation ( $\left.\dot{\mathrm{V}}_{\mathrm{I}} ; \mathrm{L} / \mathrm{min}\right)$. The pressure of end-tidal $\mathrm{P}_{\mathrm{ETCO}}$ was measured using a portable, calibrated capnograph (Masimo EMMA, Danderyd, Sweden) with a personal mouthpiece and nose clip and peripheral oxygen saturation $\left(\mathrm{SpO}_{2}\right)$ was measured with a portable finger pulse oximeter (Masimo SET® Rad-5, Danderyd, Sweden). Electrocardiography (ECG; ADI MLA2503 \& ADI FE132; lead II configuration) was utilized to derive instantaneous heart rate (HR; 1/R-R Interval in $\left.\mathrm{min}^{-1}\right)$. The protocol was carried out with participants sitting comfortably in a dark, quiet room with ear plugs and eyes closed. Resting ventilation at each altitude was analyzed from a one-minute representative period near the end of a 10-min baseline period, whereas $\mathrm{P}_{\mathrm{ET}} \mathrm{CO}_{2}$ and $\mathrm{SpO}_{2}$ measures were obtained after stability was achieved.

\section{$\underline{\text { Swallow stimulation }}$}

1. Swallows produced during the baseline respiratory data via normal saliva collection in the mouth, termed saliva swallows.

2. Water swallows were trigged via water delivery from a $250 \mathrm{~mL}$ wash bottle (Nalgene 2089-0008 Narrow-Mouth Economy Bottle; Thermo Scientific, Waltham, MA, USA) inserted approximately $5 \mathrm{~cm}$ into the participant's mouth, lateral to the pneumotachometer mouthpiece. The wash bottle was positioned by each participant to ensure comfort with the water delivery. The infusion protocol began by recording a thirty-second baseline with all instrumentation in place. Following this baseline, water was infused at $\sim 1 \mathrm{~mL} / \mathrm{second}$ for 30 seconds into the participants' mouths. Finally, a 30 second washout was conducted after all instrumentation remaining in place. In all 
instances, participants were instructed before the introduction of water to swallow normally as needed.

\section{$\underline{\text { Statistical Analysis }}$}

Data was analyzed from seven participants (5 female and 2 male) ages 19-23 at 1,045m (Calgary), 3,440m (Namche; day 3 at altitude), and 4,371m (Pheriche; day 7 at altitude) (Figure 3-1). All results were expressed as means \pm standard deviation (SD) using SPSS software (IBM).

To examine changes in swallow phase preference the following designations were used for respiratory phase: A) transition from inspiration to expiration (In-Ex); within expiration (Ex-Ex); transition from expiration to inspiration (Ex-In); and within inspiration (In-In). Then the following assigned coding system was used with $\mathrm{In}-\mathrm{Ex}=1$; Ex-Ex $=2 ;$ Ex-In $=3$; and In-In $=4$ to categorize where each swallow occurred (Table 31). Finally, Wilcoxon signed ranks tests were run to determine changes across swallowtype and altitude, as we have previously used (Pitts, Rose, Poliacek, Condrey, Davenport, \& Bolser, 2015).

Swallow apnea duration was measured as the period of zero airflow in the event of a swallow (Figure 3-2). The apnea duration then was divided into three sub-phases: a) pre-swallow apnea, b) duration of submental sEMG, and c) post-swallow apnea (Figure 3-2). Pre-swallow apnea began at the time of zero airflow before the submental activation. Submental sEMG duration was measured as the activation and inactivation of submental sEMG. Post-swallow apnea was measured as the zero airflow after the inactivation of submental complex (Figure 3-2). Additionally respiratory rate, heart rate,

mean arterial pressure (MAP), $\dot{\mathrm{V}}_{\mathrm{I}}, \mathrm{SpO}_{2}, \mathrm{P}_{\mathrm{ET}} \mathrm{CO}_{2}$ and steady-state chemoreflex drive (SS- 
CD) were measured. The SS-CD was computed by calculating a stimulus index (SI; $\mathrm{P}_{\mathrm{ETCO}} / \mathrm{SpO}_{2}$ ), and then comparing minute ventilation against SI (Bruce CD, 2018; Pfoh, Steinback, Berg, Bruce, \& Day, 2017). A repeated measures ANOVA was used to determine differences in swallow motor pattern and respiratory parameters across the three elevations with significance at $p \leq 0.05$, and if significance was met the LSD posthoc test was used. A $p \leq 0.07$ was designated as "approaching significance".

\section{Results}

Swallow was present during baseline respiratory measurements (saliva swallows), and reliably elicited with infusion of water in all subjects (water swallows). A total of 379 swallows (122 saliva and 257 water) were analyzed across the three altitudes (142 at $1,045 \mathrm{~m} ; 121$ at $3,440 \mathrm{~m}$; and 116 at 4,371m).

\section{$\underline{\text { Swallow-breathing coordination }}$}

Table 3-1 reports percent of swallow occurrences across each respiratory phase/transition. Water swallows had a strong In-Ex phase preference (69-79\%) which was maintained through the ascent protocol. For saliva swallows at $1,045 \mathrm{~m}$ only $43 \%$ occurred during In-Ex [significantly different than water $(\mathrm{Z}=-3.3, p<0.001)$ ], but this shifted at 3,440m with $76 \%$ of swallows occurring during In-Ex [significantly different than $1,045 \mathrm{~m}(\mathrm{Z}=-3.3, p<0.001)]$. At the highest altitude $4,371 \mathrm{~m}$ the percent of swallows which occurred during the In-Ex transition reduced to 55\% $(p=0.07)$. Interestingly, at $1,045 \mathrm{~m} 21 \%$ of saliva swallows occurred during inspiration (In-In), which reduced to $6 \%$ at $3,440 \mathrm{~m}$ and at $4,371 \mathrm{~m}$ none occurred. In contrast $<6 \%$ of water swallows occurred during inspiration (Table 3-1). 


\section{Change of swallow motor pattern with increasing altitude}

Figure 3-3 demonstrates changes in pre-swallow apnea, submental duration, and post swallow apnea plotted by subjects across the three altitude locations. For swallows elicited by water, the average submental duration (ms) approached significance $[1170 \pm$ $539,1038 \pm 218$, and $710 \pm 227$ respectively $\left.\left(\mathrm{F}_{2,12}=4.19, p=0.07\right)\right]$. As elevation increased pre-swallow apnea duration (ms) significantly decreased [-256 $\pm 236,-115 \pm$ 99 , and $-5 \pm 172$ respectively $\left.\left(F_{2,12}=4.218, p=0.06\right)\right]$, and post-swallow apnea duration (ms) significantly increased [56 $\pm 109,111 \pm 171$, and $\left.241 \pm 218\left(\mathrm{~F}_{2,12}=6.137, p<0.05\right)\right]$ (Table 3-2 and Figure 3-2 and 3-3). Of note, pre-swallow submental sEMG activity was seen during swallows at each elevation and of each type (Figure 3-2). For saliva swallows there was no significant change in submental sEMG and apnea duration, or swallow frequency (Table 3-2).

\section{$\underline{\text { Breathing related variables }}$}

Table 3-2 also illustrates resting minute ventilation $\left(\dot{\mathrm{V}}_{\mathrm{I}}\right)$, the pressure of end-tidal $\mathrm{P}_{\mathrm{ETCO}}$, peripheral oxygen saturation $\left(\mathrm{SpO}_{2}\right)$, stimulus index $(\mathrm{SI})$ and measurement of steady-state chemoreflex drive (SS-CD) during incremental ascent to high altitude. All variables changed in predictable ways with incremental ascent. Heart rate $[81.6 \pm 9.5$, $97.8 \pm 7.9$, and $93.5 \pm 5.8$ respectively $\left.\left(\mathrm{F}_{2,12}=10.29, p<0.05\right)\right]$, MAP $[90.4 \pm 8.4,96.0 \pm$ 6.5 , and $99.1 \pm 9.2$ respectively $\left.\left(\mathrm{F}_{2,12}=11.88, p<0.05\right)\right]$ and SS-CD significantly increased as altitude increased $[36.8 \pm 8.5,49.3 \pm 12.7$, and $58.7 \pm 19.5$ respectively $\left.\left(\mathrm{F}_{2,12}=7.41, p<0.05\right)\right] . \mathrm{SpO}_{2}\left[96.2 \pm 1.0,88.1 \pm 2.3\right.$, and $83.3 \pm 5.3$ respectively $\left(\mathrm{F}_{2,12}=\right.$ 37.44, $p<0.001)]$ and $\mathrm{P}_{\mathrm{ET}} \mathrm{CO}_{2}\left[31.1 \pm 4.2,25.9 \pm 2.7\right.$, and $21.3 \pm 2.3$ respectively $\left(\mathrm{F}_{2,12}=\right.$ 31.61, $p=0.001)$ ] significantly decreased as altitude increased. Additionally, respiratory 
rate and instantaneous minute ventilation remained stable across all elevations (Table 32).

\section{Discussion}

This is the first evidence of a significant change in swallow-breathing coordination as well as swallow production during incremental ascent to high altitude. There was a significant change in swallow phase preference comparing saliva to water swallows during baseline and approached significance at the highest elevation $(4,371 \mathrm{~m})$. This was due to a shift in the dominance of the In-Ex pattern seen during water swallows and at 3,440m for saliva swallows. Additionally, in the water trials there was a significant increase in the post-swallow apnea period and a decrease (approaching significance) in the submental duration and pre-swallow apnea, while the overall swallow apnea duration did not change.

\section{$\underline{\text { Phase Preference }}$}

Swallow phase preference has been intensely studied in humans (Martin-Harris, 2008; Martin-Harris, Brodsky, Michel, Castell, Schleicher, Sandidge, Maxwell, \& Blair, 2008; Martin-Harris, Brodsky, Price, Michel, \& Walters, 2003b; Martin-Harris \& McFarland, 2013; Pratali, Cavana, Sicari, \& Picano, 2010; Wheeler Hegland, Huber, Pitts, Davenport, \& Sapienza, 2011a; Wheeler Hegland, Huber, Pitts, \& Sapienza, 2009a), as well as in cats (Dick, Oku, Romaniuk, \& Cherniack, 1993a; Pitts, Gayagoy, Rose, Poliacek, Condrey, Musslewhite, Shen, Davenport, \& Bolser, 2015a; Pitts, Rose, Mortensen, Poliacek, Sapienza, Lindsey, Morris, Davenport, \& Bolser, 2013a; Pitts, Rose, Poliacek, Condrey, Davenport, \& Bolser, 2015), goats (Bonis, Neumueller, Marshall, Krause, Qian, Pan, Hodges, \& Forster, 2011a; Feroah, Forster, Fuentes, Lang, 
Beste, Martino, Pan, \& Rice, 2002; Feroah, Forster, Fuentes, Wenninger, Martino, Hodges, Pan, \& Rice, 2002), and rats (Saito, Ezure, \& Tanaka, 2002a, 2002b). However, all the peripheral stimulations and/or central mechanisms which regulate their interactions are not entirely understood. In the present study there was not a strong expiratory phase preference $(\sim 80 \%)$ which is observed in single swallow studies in which a 5 or $10 \mathrm{~mL}$ bolus is placed in the mouth (Wheeler Hegland, Huber, Pitts, \& Sapienza, 2009a). Saliva swallows (probably most akin to the typical single swallow task) demonstrated only $9 \%$ occurred during expiration, with $43 \%$ occurring in the transition of In-Ex, and of great interest is that $21 \%$ of these swallows occurred during inspiration (Table 3-1).

The dominance of In-Ex preference may be due in part to the mouthpiece which forces an "open mouth" swallow. It has been shown that muscle spindle afferents, in the masseter muscle, increase in discharge frequency during active opening of the jaw (Taylor, Hidaka, Durbaba, \& Ellaway, 1997). It has also been shown that input of muscle spindle afferents influence other central pattern generators [i.e. locomotion (Pearson, 1995)], and has been speculated that muscle spindle afferents influence mastication CPG output (Kolta, Lund, \& Rossignol, 1990; Lund, 2011). This information allows speculation that position of the jaw, indicated by proprioception of muscle spindle afferents can modulate the interaction between the swallow and breathing CPGs.

These changes could also be related to the effects of hypoxia and/or hypocapnia on swallow. Although there are limited studies, there are also conflicting results. In mice an increase in swallow frequency was reported (Khurana \& Thach, 1996), no change in rat (Ghannouchi, Duclos, Marie, \& Verin, 2013), and a decrease in the cat (Nishino, 
Kohchi, Honda, Shirahata, \& Yonezawa, 1986). Hypoxia has also been studied in nonnutritive swallow in newborn lambs which showed a decrease in frequency during quiet sleep (Duvareille, Lafrance, Samson, St-Hilaire, Pladys, Micheau, Bournival, Langlois, \& Praud, 2007). Interestingly, hypercapnia shifts swallows towards In and ExIn (D’Angelo, Diaz-Gil, Nunn, Simons, Gianatasio, Mueller, Meyer, Pierce, Rosow, \& Eikermann, 2014) while we found that hypocapnia with hypoxia shifts swallow toward In-Ex. In light of the present data, further studies may need to investigate swallowbreathing coordination not only with variation of respiratory drive but swallow drive as well. We speculate that the water trials increased swallow excitability, which likely altered and stabilized its relationship with breathing.

\section{$\underline{\text { Swallow motor pattern }}$}

In contrast to the swallow-breathing coordination data, the largest changes in the swallow motor pattern with ascent were on the water swallows, with a $39 \%$ decrease in the submental duration (Figure 2-3) at the highest altitude (compared to Calgary). This effect has been demonstrated in cats when swallow was coordinated with cough (airway irritation discussed below) (Leow, Huckabee, Sharma, \& Tooley, 2006); however we could find no study demonstrating a decrease in submental sEMG in healthy adults when using a mechanical/cold stimulus on the back of the mouth (Sciortino, Liss, Case, Gerritsen, \& Katz, 2003) or altering oral stimulation with taste (Leow, Huckabee, Sharma, \& Tooley, 2006).

To protect the airway during the pharyngeal phase of swallow the vocal folds must be adducted (zero flow; swallow apnea) during the laryngeal exposure to the bolus (Butler, Postma, \& Fischer, 2004; Chi-Fishman \& Sonies, 2000; Ding, Logemann, Larson, \& 
Rademaker, 2003; Kijima, Isono, \& Nishino, 1999; Martin-Harris, Brodsky, Price, Michel, \& Walters, 2003b; Martin, Logemann, Shaker, \& Dodds, 1994a; Paydarfar, Gilbert, Poppel, \& Nassab, 1995; Wheeler Hegland, Huber, Pitts, Davenport, \& Sapienza, 2011a). In a review by Martin-Harris (2008), she stated that increases in the timing from the onset of the submental activity to the apnea period is related to significant clinical risk for aspiration. Evidence of this has been demonstrated in patients with Parkinson's disease with dysphagia (Ertekin, 2014). Based on this current data, we speculate that the decrease in submental sEMG and the shift in its activity to closer to the start of the swallow apnea period could increase airway protection. Of note, Ertekin and colleagues $(2014 ; 2013)$ demonstrated an activation of the submental complex during the preswallow respiratory phase that is likely related to infusion of water into the mouth (termed foreburst). Figure 3-2 demonstrates the difference between swallow-related and pre-swallow submental activity.

\section{Airway Irritation}

Exposure to high altitude conditions is also associated with airway irritation from dry air and insensible water loss, which results in a chronic cough (Freer, 2004). The most common diagnosis in the Nepal Himalaya is "Khumbu cough", also known as "high altitude hack" (Freer, 2004), thought to be caused by dry air, sub-zero temperatures, dust, and exposure to yak dung stoves in the lodges (Linoby, Nias, Ahmad, Zaki, Canda, Sariman, Azam, \& Amat, 2013). There is evidence that dry air increases airway responsiveness (Van Oostdam, Walker, Knudson, Dirks, Dahlby, \& Hogg, 1986), and prolonged exposure results in an inflammatory response, desquamation of the epithelium, and edema of submucosa (Florey, Carleton, \& Wells, 1932). While each subject did have 
evidence of coughing across the recording period, none were actively coughing during the measurement period. It is possible that activation of irritant receptors can alter swallow production without cough as a presenting feature.

\section{$\underline{\text { Respiratory Drive }}$}

The changes in swallow and swallow-breathing coordination were also accompanied by changes/adaption of the chemoreflexes driving breathing. It is known that these reflexes become more dynamic as individuals acclimatize to their respective environment (Pfoh, Steinback, Berg, Bruce, \& Day, 2017) (Steinback \& Poulin). To asses this adaptation, Pfoh and colleagues (2017) created an index of steady-state chemoreflex drive (SS-CD), taking into account resting ventilation indexed against the overall contributions of both low $\mathrm{O}_{2}$ and low $\mathrm{CO}_{2}$ during exposure to hypoxia. Based on the magnitude of this index the significant change in the SS-CD from $1,045 \mathrm{~m}$ to $3,440 \mathrm{~m}$ is evidence of respiratory acclimatization in our participants [see also (Huang, Alexander, Grover, Maher, McCullough, McCullough, Moore, Sampson, Weil, \& Reeves, 1984)]. Blood levels of $\mathrm{O}_{2}$ and $\mathrm{CO}_{2}$ are maintained in part by central (brainstem) and peripheral (carotid body) chemoreceptors. Central chemoreceptors, located throughout brainstem, detect $\mathrm{PCO}_{2} /\left[\mathrm{H}^{+}\right]$accumulation (Guyenet \& Bayliss, 2015). Peripheral chemoreceptors located bilaterally within carotid bodies detect rapid changes in both $\mathrm{O}_{2}$ and $\mathrm{CO}_{2}$ synergistically (Fitzgerald \& Parks, 1971; Lahiri \& DeLaney, 1975; LópezBarneo, González-Rodríguez, Gao, Fernández-Agüera, Pardal, \& Ortega-Sáenz, 2016). A primary location for integrating these signals is in the nucleus tractus solitarius (NTS) (Jordan \& Spyer, 1986; Paton, Deuchars, Li, \& Kasparov, 2001). Due to the overlap in sensory integration in the NTS for breathing and swallow (Jean, 1984a, 2001b), this may 
be a site of shared central excitability which affects both respiratory and swallow central pattern generators.

\section{$\underline{\text { Clinical Implications }}$}

Altitude exposure has inherent risks with 1-2\% experiencing high altitude pulmonary edema (HAPE) (Houston, 1960; Hultgren, 1969; Schoene, Hackett, Henderson, Sage, Chow, Roach, Mills, \& Martin, 1986), a form of high altitude sickness, and of those $65 \%$ are diagnosed with a concomitant respiratory infection (most commonly pneumonia) (Leshem, Pandey, Shlim, Hiramatsu, Sidi, \& Schwartz, 2008). It would be of interest to know if climbers with pneumonia display the same adaptations in swallow, especially in light of our knowledge of pneumonia rates with dysphagia.

\section{Conclusion}

Our results suggest that there are changes in swallow-breathing coordination and swallow motor production that increase airway protection with incremental ascent to high altitude. In conclusion, we suspect the adaptive changes in swallow were likely due to the exposure to superimposed hypoxia and hypocapnia, along with the increased airway irritation. 


\section{Table 3-1}

Percent of swallow occurrence during breathing across the three levels of ascent

\begin{tabular}{rcccc}
\hline & In-Ex & Ex-Ex & Ex-In & In-In \\
\hline Saliva Swallow & & & & \\
$1,045 \mathrm{~m}$ & 43 & 9 & 27 & 21 \\
$3,440 \mathrm{~m}$ & 76 & 12 & 6 & 6 \\
$4,371 \mathrm{~m}$ & 55 & 15 & 30 & 0 \\
Water Swallow & & & & \\
$1,045 \mathrm{~m}$ & 79 & 9 & 9 & 2 \\
$3,440 \mathrm{~m}$ & 69 & 14 & 11 & 6 \\
$4,371 \mathrm{~m}$ & 76 & 11 & 11 & 2 \\
\hline
\end{tabular}

${ }^{2}$ Reprinted with permsisson from Huff, Day, English, Reed, Zouboules, Saran, Leacy, Mann, Peltonen, O'Halloran, Sherpa and Pitts. Swallow-breathing coordination during incremental ascent to altitude. Respiratory Physiology \& Neurobiology, 2018. (Table 1) 


\section{Table 3-2}

$\underline{\text { Means, standard deviations (SD), and } p \text {-values comparing ventilatory, cardiac and }}$ $\underline{\text { acclimation values, as well as saliva and water swallows at the three different elevations }}$ are shown in this table.

\begin{tabular}{|c|c|c|c|c|c|c|c|c|c|c|}
\hline & \multicolumn{3}{|c|}{ Calgary $(1,045 m)$} & \multicolumn{3}{|c|}{ Namche $(3,440 \mathrm{~m})$} & \multicolumn{3}{|c|}{ Pheriche $(4,371 \mathrm{~m})$} & \multirow{2}{*}{$p$-values } \\
\hline & Mean & \pm & SD & Mean & \pm & SD & Mean & \pm & SD & \\
\hline Resting RR $\left(\mathrm{min}^{-1}\right)$ & 14.0 & \pm & 6.0 & 15.2 & \pm & 3.9 & 15.5 & \pm & 5.2 & 0.42 \\
\hline Resting Heart Rate & 81.6 & \pm & 9.5 & 97.8 & \pm & $7.9 * * *$ & 93.5 & \pm & $5.8 * *$ & 0.004 \\
\hline MAP $(\mathrm{mm} \mathrm{Hg})$ & 90.4 & \pm & 8.4 & 96.0 & \pm & $6.5 * *$ & 99.1 & \pm & $9.2 * * *$ & 0.004 \\
\hline $\mathrm{SpO}_{2}(\%)$ & 96.2 & \pm & 1.0 & 88.1 & \pm & $2.3 * * *$ & 83.3 & \pm & $5.3 * * * \dagger \dagger$ & $<0.001$ \\
\hline $\mathrm{P}_{\mathrm{ET}} \mathrm{CO}_{2}$ (Torr) & 31.1 & \pm & 4.2 & 25.9 & \pm & $2.7 * *$ & 21.3 & \pm & $2.3 * * *+\dagger \dagger$ & 0.001 \\
\hline$\dot{V}_{I}(\mathrm{~L} / \mathrm{min})$ & 11.9 & \pm & 2.7 & 14.2 & \pm & 2.6 & 14.7 & \pm & 3.8 & 0.07 \\
\hline $\mathrm{SS}-\mathrm{CD}\left(\mathrm{V}_{\mathrm{I}} / \mathrm{SI}\right)$ & 36.8 & \pm & 8.5 & 49.3 & \pm & $12.7 *$ & 58.7 & \pm & $19.5 * * \dagger$ & 0.02 \\
\hline \multicolumn{11}{|l|}{ Saliva Swallow Data } \\
\hline Submental Duration (ms) & 1480 & \pm & 804 & 1070 & \pm & 490 & 1015 & \pm & 457 & 0.35 \\
\hline Swallow Apnea Duration (ms) & 1088 & \pm & 433 & 1121 & \pm & 253 & 1010 & \pm & 374 & 0.77 \\
\hline Pre-Swallow Apnea (ms) & -296 & \pm & 220 & -183 & \pm & 113 & -141 & \pm & 265 & 0.45 \\
\hline Post-Swallow Apnea (ms) & -99 & \pm & 363 & 233 & \pm & 385 & 174 & \pm & 101 & 0.30 \\
\hline \multicolumn{11}{|l|}{ Water Swallow Data } \\
\hline Submental Duration (ms) & 1170 & \pm & 539 & 1038 & \pm & 218 & 710 & \pm & $227 * \dagger \dagger \dagger$ & 0.07 \\
\hline Swallow Apnea Duration (ms) & 973 & \pm & 398 & 1030 & \pm & 165 & 946 & \pm & 285 & 0.60 \\
\hline Pre-Swallow Apnea (ms) & -256 & \pm & 236 & -115 & \pm & 99 & -5 & \pm & $126 *$ & 0.06 \\
\hline Post-Swallow Apnea (ms) & 56 & \pm & 109 & 111 & \pm & 171 & 241 & \pm & $218 * * \dagger$ & 0.02 \\
\hline
\end{tabular}

Reported $p$-values are for repeated measures oneway ANOVA and significant values are bolded

* Significant difference from Calgary $* p \leq 0.06, * * p<0.05, * * * p<0.01$

$\uparrow$ Significant difference between Namche and Pheriche ${ }^{\dagger} p \leq 0.06,{ }^{\dagger \dagger} p<0.05,{ }^{\dagger \dagger} p<0.01$

Resting respiratory rate (RR), mean arterial pressure (MAP), peripheral O2 saturation (SpO2), end tidal $\mathrm{CO} 2$ pressure (PETCO2), instantaneous minute ventilation ( $\dot{\mathrm{V} I})$, and steady-state chemoreflex drive (SS-CD) are recorded. Submental (swallow) duration, swallow apnea duration, pre-swallow apnea and post-swallow apnea (Figure 3-2) are recorded in both saliva and water conditions. Figure 3-3 displays swallow data by participant.

${ }^{2}$ Reprinted with permsisson from Huff, Day, English, Reed, Zouboules, Saran, Leacy, Mann, Peltonen, O'Halloran, Sherpa and Pitts. Swallow-breathing coordination during incremental ascent to altitude. Respiratory Physiology \& Neurobiology, 2018. (Table 2) 
${ }^{2}$ Figure 3-1. Timeline of travel, ascent, and recording locations.

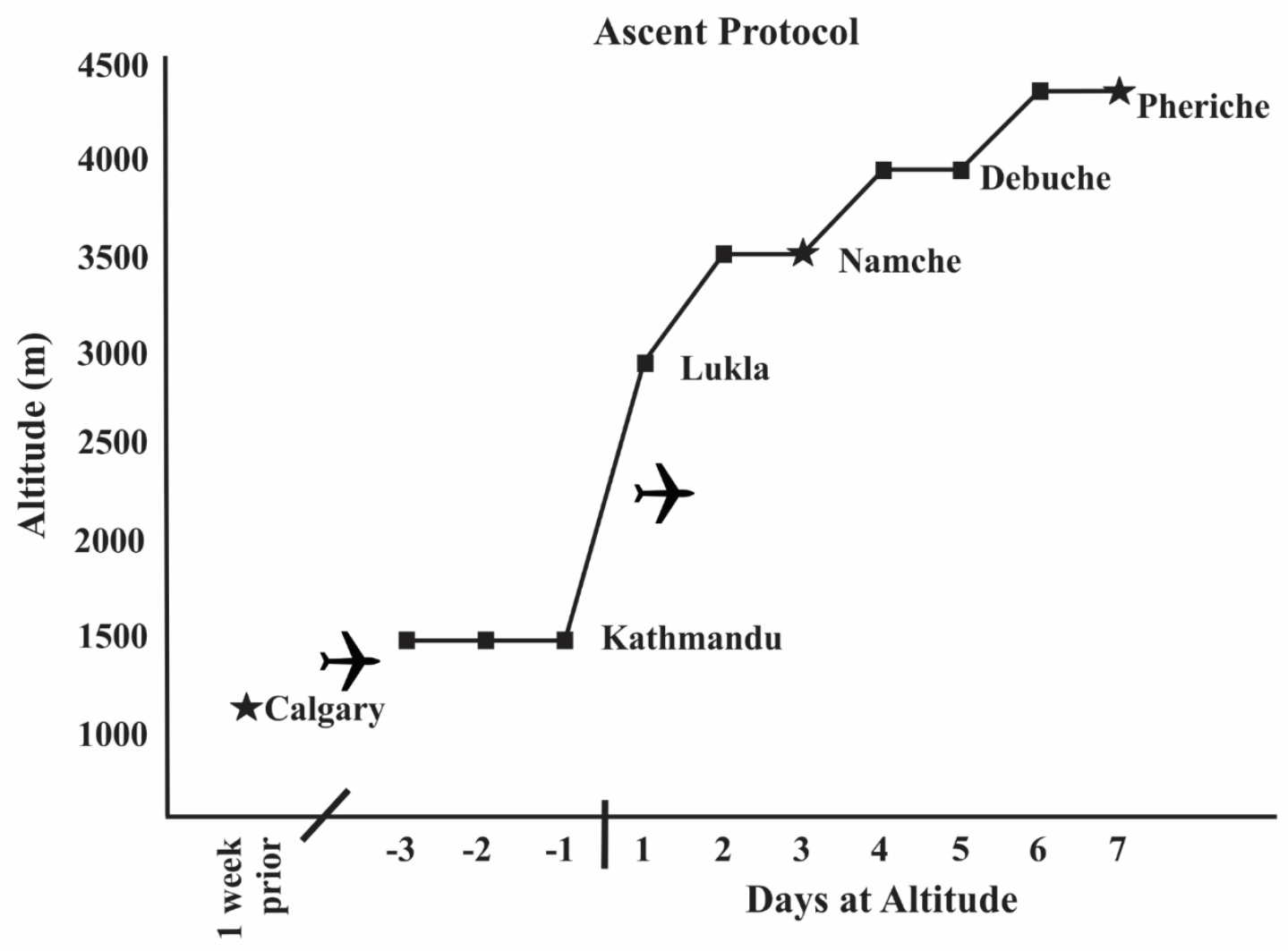

The $(\star)$ represents where data was collected, and ( $\downarrow)$ indicates flights.

${ }^{2}$ Reprinted with permsisson from Huff, Day, English, Reed, Zouboules, Saran, Leacy, Mann, Peltonen, O'Halloran, Sherpa and Pitts. Swallow-breathing coordination during incremental ascent to altitude. Respiratory Physiology \& Neurobiology, 2018. (Figure 1) 
${ }^{2}$ Figure 3-2. Example of submental sEMG and airflow from the same participant from

Calgary $(1,045 \mathrm{~m})$ and Pheriche $(4,371 \mathrm{~m})$ during the water swallow protocol.

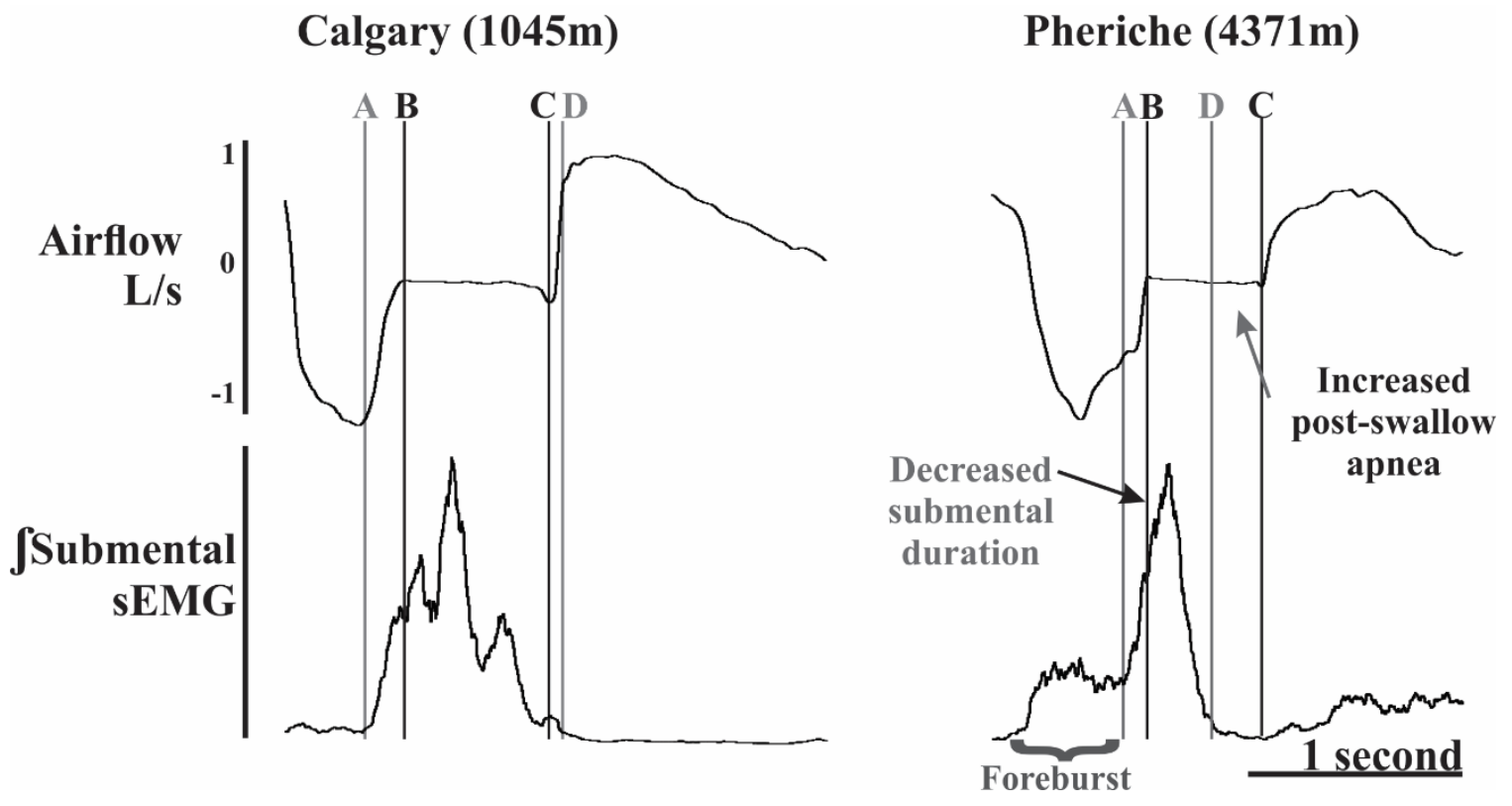

$\mathrm{B}$ to $\mathrm{C}$ marks the swallow apnea period. A to $\mathrm{B}$ is the pre-swallow submental activity, $\mathrm{A}$ to $\mathrm{D}$ is the submental duration and $\mathrm{C}$ to $\mathrm{D}$ is the post-swallow apnea period. At $4,371 \mathrm{~m}$, there was a significant increase in the post-swallow apnea as well as a decrease submental duration. The "foreburst" is activity related to water being introduced to the oral cavity.

${ }^{2}$ Reprinted with permsisson from Huff, Day, English, Reed, Zouboules, Saran, Leacy, Mann, Peltonen, O’Halloran, Sherpa and Pitts. Swallow-breathing coordination during incremental ascent to altitude. Respiratory Physiology \& Neurobiology, 2018. (Figure 2) 
${ }^{2}$ Figure 3-3. Scatter plot of duration measures (pre-swallow, submental and post-swallow) for each subjects across the recording locations for the saliva (A) and water (B) swallow tasks.
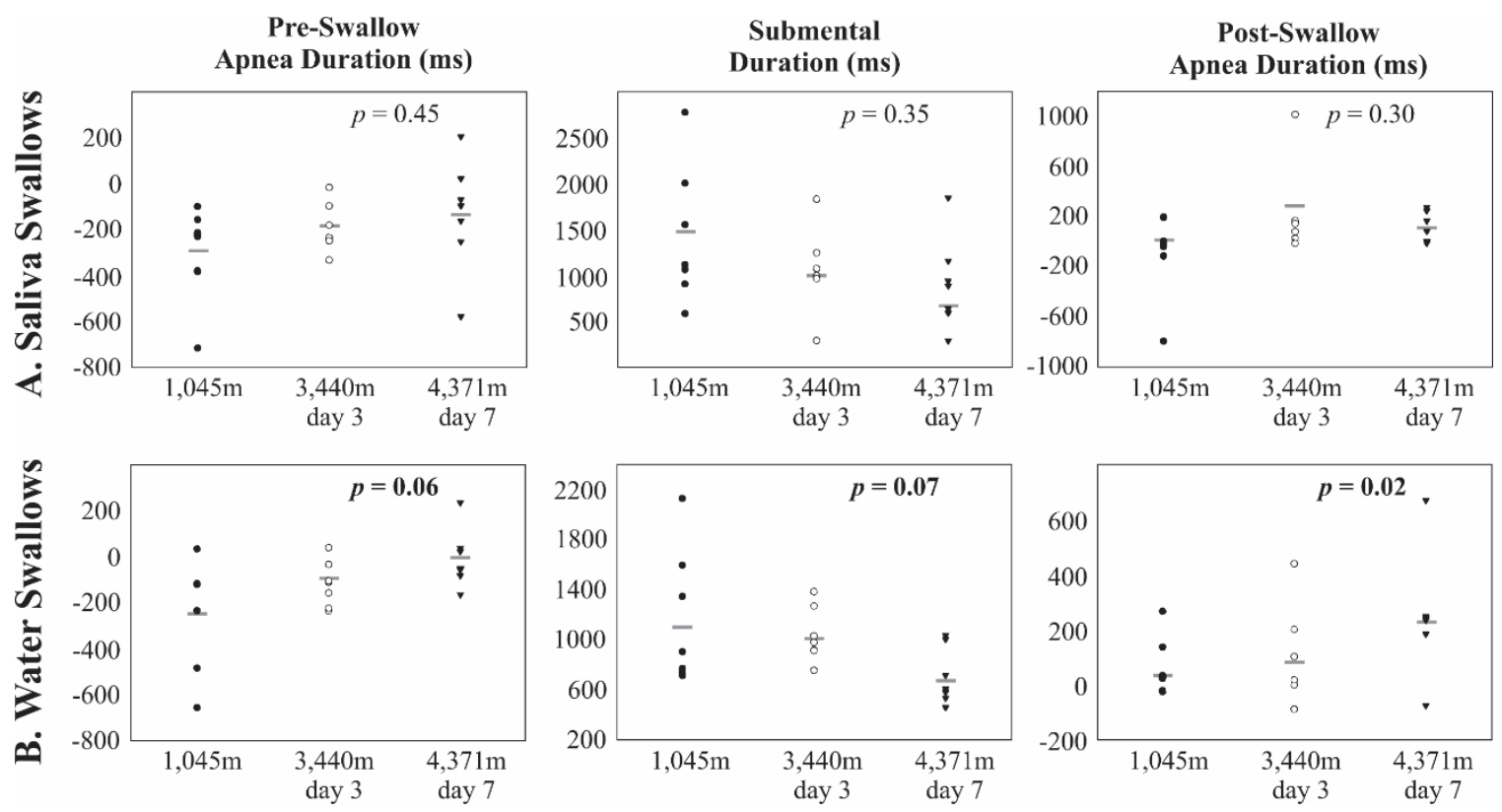

Repeated measures ANOVA p-value reported for each dependent measure, and gray line represents group mean.

${ }^{2}$ Reprinted with permsisson from Huff, Day, English, Reed, Zouboules, Saran, Leacy, Mann, Peltonen, O'Halloran, Sherpa and Pitts. Swallow-breathing coordination during incremental ascent to altitude. Respiratory Physiology \& Neurobiology, 2018. (Figure 3) 


\title{
CHAPTER 4
}

\section{SEX SPECIFIC VAGAL AND SPINAL MODULATION OF BREATHING WITH CHEST COMPRESSION (PART 1)}

\author{
Introduction \\ Lung volume is modulated by sensory afferent feedback transmitted through both \\ vagal and spinal pathways. Vagal sensory feedback from pulmonary stretch receptors \\ (PSRs) relays to the ventrolateral nucleus of the Tractus Solitaris (NTS) in the brainstem \\ (Berger, 1977). Muscle spindles and Golgi tendon organs in respiratory muscles such as \\ intercostals and joint receptors of the ribcage (Zechman Jr \& Wiley, 2011) send \\ proprioceptive information through spinal nerves, via ascending pathways, to respiratory \\ centers in the medulla (Lust, 2007). \\ PSRs rapidly respond to mechanical stimuli (tracheal/bronchial stretch) during \\ lung inflation (Widdicombe, 2003). As the lungs expand, PSR activity increases, driving \\ pump cell activity in the NTS, ultimately resulting in inhibition of the ongoing inspiratory \\ phase. During rapid inflation this is known as the Hering-Breuer Reflex (Baertsch, \\ Baertsch, \& Ramirez, 2018; Davenport \& Wozniak, 1986). It has also been shown that \\ vagotomy, which eliminates inputs from PSRs and other pulmonary afferents, \\ significantly reduces respiratory rate by prolonging expiration and also reduces \\ variability (Baertsch, Baertsch, \& Ramirez, 2018).
}


Lung volume is also regulated by spinal sensory feedback from proprioceptive receptors in the thoracic cavity (Campbell \& Howell, 1962; Lust, 2007). Changes in chest wall volume and pressure provide information about muscle length-tension relationships (Campbell \& Howell, 1962; Zechman Jr \& Wiley, 2011), and stretch receptors located in the thoracic cavity indirectly monitor lung inflation (Lust, 2007). The primary function of the intercostal muscles is to mechanically maintain proper rib placement (De Troyer, Kirkwood, \& Wilson, 2005; Feldman, 1986) and stability during movement. Their activity is highly modulated by feedback from their muscle spindles, Golgi tendon organs, and other joint receptors (D'Angelo, 1982; De Troyer, 1997; Jaiswal \& Davenport, 2016). Compared to caudal, rostral ribs contain a higher density of mechanoreceptors and increased movement-related activation during breathing (D'Angelo, 1982).

Vagotomy and chest compression have been studied in several species including dog, cat (Remmers, 1970; Shannon, 1975; Shannon, 1979b), rabbit (D'Angelo, Miserocchi, \& Agostoni, 1976; GARCIA, 1959), and human (Agostoni, D'angelo, Torri, \& Ravenna, 1977; Bland, Lazerou, Dyck, \& Cherniack, 1967). Across species, chest compression decreases tidal volume and inspiratory and expiratory duration; vagotomy has differential effects. In humans, chest compression increases respiratory rate and reduces tidal volume (Bland, Lazerou, Dyck, \& Cherniack, 1967). Proprioceptive feedback alters respiratory drive during loading (e.g. internal or external mechanical, elastic, or resistance loading). Work by Bolser and Shannon and colleagues (Bolser, Lindsey, \& Shannon, 1983, 1984; Bolser, Lindsey, \& Shannon, 1988; Bolser \& Remmers, 1989; Shannon, 1980; Shannon, Bolser, \& Lindsey, 1987) demonstrates that 
activation of intercostal afferents (via Golgi tendon organs) strongly inhibits medullary inspiratory neurons, resulting in decreases in diaphragm, intercostal, and laryngeal inspiratory muscle activity (Shannon, Bolser, \& Lindsey, 1987).

Respiration has the unique responsibility of maintaining gas exchange regardless of environmental demands and/or execution of other behaviors such as swallow. The full understanding of how the totality of afferent feedback alters respiratory regulation is unknown, particularly in the rat. Additionally, the variability of the available data suggests that there are unknown features, which are important to its regulation (e.g. sex). The purpose of this study was to systematically alter afferent feedback before and during a mechanical challenge (chest compression). We tested the hypothesis that selective inhibition of PSRs, vagotomy, or lidocaine administration to the pleural space would produce different effects on breathing during chest compression. Additionally, we predicted that females would have a more pronounced response, due to less advantageous chest wall geometry and a higher contribution of the chest wall to rest breathing.

\section{Methods}

Experiments were performed on 43 anesthetized spontaneously breathing Sprague Dawley $(\mathrm{SpD})$ retired breeder rats $[24$ male $(0.49 \pm 0.04 \mathrm{~kg})$ and 19 female $(0.39 \pm$ $0.08 \mathrm{~kg})]$. Protocol was approved by University of Louisville Institutional Animal Care and Use Committee (IACUC). The animals were initially anesthetized with gaseous isoflurane $\left(1.5-2 \%\right.$ with $\left.100 \% \mathrm{O}_{2}\right)$ while a femoral intravenous (i.v.) cannula was placed for administration of sodium pentobarbital ( $25 \mathrm{mg} / \mathrm{kg}$, i.v.). Isoflurane was discontinued and supplementary doses of sodium pentobarbital were administered as needed 
throughout the experiment. Anesthetic level was evaluated by withdrawal reflex of the forelimb and hindlimb and licking in response to oral water administration. A dose of atropine sulfate $(0.01 \mathrm{mg} / \mathrm{kg}$, i.v. $)$ was given at the beginning of the experiment to reduce secretions from repeated tracheal stimulation. Following administration of atropine sulfate, a tracheostomy was performed and followed by incision of the esophagus for placement of a 20 gauge catheter to measure esophageal pressure. Body temperature was maintained using a heating pad.

Electromyograms (EMG) of multiple respiratory-related muscles were recorded using bipolar insulated fine wire electrodes according to the technique of Basmajian and Stecko (Basmajian \& Stecko, 1962). The costal diaphragm (sternal) along with the thyroarytenoid muscle (primary laryngeal adductor) were used to evaluate breathing. The thyroarytenoid electrodes were inserted through the cricothyroid window into the anterior portion of the vocal folds, which were visually inspected post-mortem. For electrode placement of the costal diaphragm, palpation and elevation of the xyphoid process was followed by insertion of a needle directed caudally, and the needle was hooked underneath the xyphoid process near the costal diaphragm muscle attachment. Electrodes were placed bilaterally into the pectoralis muscle to record electrocardiogram (ECG) activity and to remove heart artifact from EMG traces.

\section{Experimental Protocol}

Three experimental protocols were performed on three cohorts of male and female SpD rats. A) An extra-thoracic vagotomy was performed in $12 \mathrm{SpD}$ rats [6 male $(0.48 \pm 0.03 \mathrm{~kg})$ and 6 female $(0.35 \pm 0.06 \mathrm{~kg})] . \mathrm{B})$ Lidocaine $(10 \%)$ was nebulized into the trachea in $13 \mathrm{SpD}$ rats $[8$ male $(2$ sham $)(0.40 \pm 0.03 \mathrm{~kg}), 5$ female $(2$ sham $)(0.39 \pm$ 
$0.09 \mathrm{~kg})]$. C) Lidocaine (10\%) was injected into the pleural space in $18 \mathrm{SpD}$ rats [10 male (2 sham) $(0.46 \pm 0.05 \mathrm{~kg}), 8$ female $(2$ sham $)(0.41 \pm 0.08 \mathrm{~kg})]$.

\section{Removal/reduction of vagal feedback}

To remove all vagal afferent feedback, bilateral vagotomy at the level of the extra-thoracic trachea was performed on male and female rats in the supine position. The vagus nerves were dissected away from the sympathetic nerves and common carotid arteries. Silk suture (5-0) was looped around each vagus nerve with hemostat forceps clamped onto the suture ends for quick access after control trials had been completed. While lifting the suture attached to the hemostats, the vagus nerves were cut using spring scissors at the level of the $5^{\text {th }}-6^{\text {th }}$ tracheal ring. After bilateral vagotomy an inflation test was performed: $4 \mathrm{cc}$ of air was drawn into a $5 \mathrm{cc}$ syringe and quickly infused into the endotracheal tube to assure removal of PSR (lung volume) feedback. The order of the cuts were randomized (left vs right) across animals.

To selectively reduce vagal feedback from pulmonary stretch receptors, $10 \%$ lidocaine was nebulized into the trachea with the animal in the supine position. Using a compressor nebulizer (StrongHealth; particle size $0.5-5 \mu \mathrm{m}$; average nebulization rate 0.2 $\mathrm{mL} / \mathrm{min}$ ), 10\% Lidocaine (Cat No. L5647, Sigma-Aldrich) mixed with 2\% Evans Blue Dye (EBD, Cat No. E2129, Sigma-Aldrich) was nebulized for 15 minutes. Ten minutes after the completion of the nebulization, we performed an inflation test by injecting 4cc of air into the trachea. If the Hering-Breuer reflex was maintained (i.e. termination of inspiration followed by prolonged expiration), the animal then received an additional 5 minutes of nebulized lidocaine and was retested. This procedure was performed as 
necessary until the reflex was abolished. The addition of the dye allowed for post-mortem verification that the lidocaine penetrated the lung tissue and the intra- and extra-thoracic trachea. To minimize contamination of the lidocaine and dye into the air, a portable fume evacuation machine hovered over the mouthpiece of the nebulizer. To minimize contamination around the trachea, Vaseline-coated gauze was placed below and above the trachea, which covered any exposed area of the animal and blocked any potential absorption of lidocaine into the upper airway that was not specifically targeted by nebulized lidocaine.

\section{Reduction of spinal feedback}

To reduce spinal feedback, bilateral injections of $10 \%$ lidocaine mixed with $2 \%$ EBD were administered into the pleural space using methods from Mantilla et al. (2009). Animals were stabilized on their side while the rib cage was palpated to identify the fifth intercostal space, and the injection site was located and marked by a permanent marker, by measuring one inch rostral to the xyphoid process and moving laterally to the axial side of the rib. This was repeated on each axial side of the animal. At this location the skin was removed using skin scissors, and $20 \mu \mathrm{l}$ of lidocaine/EBD mixture was injected bilaterally using a 100- $\mu$ l Hamilton syringe with a 35 gauge beveled needle inserted 6 $\mathrm{mm}$. After both injections were complete, the animal was returned to supine position, and after a 5 minute waiting period an inflation test was performed to confirm that a reflex response was present, indicating that the lidocaine had not reached the PSRs or altered any other vagal afferent feedback.

Stimuli 
Chest compression stimuli were performed during control conditions (before lidocaine or vagotomy interventions) and also after interventions. Chest compression of the thoracic cavity was performed by placing a 2-inch thick Velcro band to restrict chest movement to the target of end-expiration of tidal volume. In order to monitor movement of the chest wall, an in-house produced piezoelectric chest strap made from a piezoelectric sensor inside a fire alarm and an elastic band (1/2 inch) mounted on an aluminum plate was loosely strapped around the chest of the animal rostral to the Velcro restriction band. This piezoelectric chest strap allowed us to observe the change in movement as a result of the restrictive band. Video was also taken for visual observation of the reduction in chest movement.

\section{$\underline{\text { Analysis }}$}

All EMG signals were amplified and filtered (100-1000 Hz). Signals were rectified and integrated (20ms) using Spike2 (Cambridge Electronic Design; Cambridge, England). EMG amplitude measures were calculated as a percent of maximum during the control period. Breathing phase durations were measured across 30 seconds of eupnea, using diaphragm EMG activity. Inspiration (I) was classified as the onset of the diaphragm activation to the peak of the diaphragm burst; "yield" was classified as the peak of the diaphragm burst to the offset of the diaphragm activation; expiration (E) was classified as the offset of diaphragm activation to the following diaphragm burst onset; thyroarytenoid activity was classified as the onset of the thyroarytenoid during expiration to the offset of thyroarytenoid (Figure 4-1); total respiratory cycle duration (TRC) was classified as the onset of the diaphragm activation to the following diaphragm burst onset (Figure 4-2); and yield was classified as the duration of post-inspiration diaphragm 
activity (Figure 4-1 and 4-2); and E1 was classified as the duration of the thyroarytenoid (laryngeal adductor) during expiration (Figure 4-1 and 4-2). Eupneic periods during the control period and the control chest compression conditions were averaged separately for male $(n=24)$ and female $(n=19)$ groups. Respiratory rate $(R R)$ was calculated as the number of respiratory cycles during a 30 second period, multiplied by 2 . EMG amplitude data is presented as $\%$ of maximum in the control period, to normalize the signal across animals.

Results are expressed as means \pm standard deviation (SD). Paired and independent t-tests and 2-way ANOVA were used as appropriate to statistically identify differences using SPSS statistical software (IBM Corporation). Analyses were made within groups (male and female) and between groups (male vs female). A difference was considered significant if the $p$-value was less than 0.05 .

\section{Results}

Significant changes are described below; all data are presented in Tables 4-1, -2, and -3 .

\section{Chest Compression under control conditions (Table 4-1)}

Comparing control eupnea to chest compression, female rats demonstrated a $22 \%$ increase in diaphragm EMG activity $(p<0.03)$, and a significant decrease in yield (early expiratory activity) duration. B) In males: E duration significantly decreased (604 \pm $172 \mathrm{~ms}$ to $\left.518 \pm 182 \mathrm{~ms}, \mathrm{t}_{23}=3.0, p<0.01\right) ;$ TRC duration significantly decreased $(824 \pm$ $163 \mathrm{~ms}$ to $720 \pm 172 \mathrm{~ms}, \mathrm{t}_{23}=3.6, p<0.01$ ); this resulted in a significant increase in $\mathrm{RR}$ $\left(74 \pm 13\right.$ to $\left.82 \pm 17, \mathrm{t}_{23}=-3.0, p<0.01\right)$. 


\section{$\underline{\text { Vagotomy }}$}

After vagotomy (Table 4-2a), female animals had a significant increase in E duration $\left(849 \pm 408 \mathrm{~ms}\right.$ to $\left.1245 \pm 444 \mathrm{~ms}, \mathrm{t}_{5}=-3.5, p<0.02\right)$, which increased TRC $(1050$ $\pm 357 \mathrm{~ms}$ to $\left.1647 \pm 489 \mathrm{~ms}, \mathrm{t}_{5}=-3.8, p<0.02\right)$ and decreased $\mathrm{RR}\left(62 \pm 22\right.$ to $40 \pm 15, \mathrm{t}_{5}=$ 2.7, $p<0.05)$; this was not true for male animals. There was also a non-significant trend towards increase in I duration for males $\left(134 \pm 37 \mathrm{~ms}\right.$ to $\left.184 \pm 38 \mathrm{~ms}, \mathrm{t}_{5}=-2.5, p=0.06\right)$ and females $\left(124 \pm 72 \mathrm{~ms}\right.$ to $\left.274 \pm 100 \mathrm{~ms}, \mathrm{t}_{5}=-2.3, p=0.07\right)$.

With the addition of chest compression (Table 4-3a), vagotomized female animals had a significant increase in I duration $\left(107 \pm 15 \mathrm{~ms}\right.$ to $\left.221 \pm 70 \mathrm{~ms}, \mathrm{t}_{5}=-3.5, p<0.02\right)$. Yield (early expiratory activity) duration increased in both males (93 $\pm 16 \mathrm{~ms}$ to $147 \pm$ $\left.31 \mathrm{~ms}, \mathrm{t}_{5}=-5.9, p<0.01\right)$ and females $\left(78 \pm 14 \mathrm{~ms}\right.$ to $\left.154 \pm 50 \mathrm{~ms}, \mathrm{t}_{5}=-3.4, p=0.02\right)$.

\section{$\underline{\text { Nebulized lidocaine }}$}

After lidocaine nebulization (Table 4-2b), there was no change in diaphragm amplitude, I, yield, E, TRC or RR in either males or females. Female sham animals had a significant decrease in E duration $\left(527 \pm 110 \mathrm{~ms}\right.$ to $\left.439 \pm 105 \mathrm{~ms}, \mathrm{t}_{1}=25.7, p=0.03\right)$.

In male animals with chest compression (Table 4-3b), the percent of maximum diaphragm EMG amplitude increased by $39 \%(p=0.01)$ following nebulization of lidocaine.

\section{$\underline{\text { Altered afferent feedback by pleural administration of lidocaine }}$}

After injection of lidocaine into the pleural space (Table 4-2c), females had a nonsignificant increase in I duration $\left(142 \pm 49 \mathrm{~ms}\right.$ to $237 \pm 134 \mathrm{~ms}, \mathrm{t}_{5}=-2.4, p=0.06$, Table 4-2c), while males had a significant decrease in E duration $(614 \pm 251 \mathrm{~ms}$ to $468 \pm 171 \mathrm{~ms}$, $\left.\mathrm{t}_{5}=2.8, p<0.03\right)$. 
In female animals with chest compression (Table 4-3c), pleural lidocaine administration produced an $85 \%$ increase in diaphragm EMG amplitude $(p<0.05)$, an increase in I duration $\left(124 \pm 30 \mathrm{~ms}\right.$ to $\left.186 \pm 53 \mathrm{~ms}, \mathrm{t}_{5}=-3.3, p<0.05\right)$, and a decrease in $\mathrm{E}$ duration ( $638 \pm 457 \mathrm{~ms}$ to $\left.353 \pm 277 \mathrm{~ms}, \mathrm{t}_{5}=3.2, p<0.05\right)$; this resulted in an increase in $\mathrm{RR}\left(81 \pm 25\right.$ to $\left.103 \pm 32, \mathrm{t}_{5}=-3.0, p<0.05\right)$.

\section{$\underline{\text { Sex differences }}$}

In control conditions, female animals had significantly longer E duration (694 \pm $394 \mathrm{~ms}$ to $\left.604 \pm 172 \mathrm{~ms}, \mathrm{~F}_{1}=4.0, p=0.05\right)$, thyroarytenoid $(T A) E M G$ duration $(782 \pm$ $457 \mathrm{~ms}$ to $\left.604 \pm 194 \mathrm{~ms}, \mathrm{~F}_{1}=5.7, p=0.02\right)$, TRC duration $(936 \pm 384 \mathrm{~ms}$ to $824 \pm 163 \mathrm{~ms}$, $\left.\mathrm{F}_{1}=4.9, p=0.03\right)$, and a slower $R R\left(70 \pm 19\right.$ to $\left.74 \pm 13, \mathrm{~F}_{1}=2.7, p=0.02\right)$ compared to males. Vagotomy enhanced these differences. $E$ duration $(1245 \pm 444 \mathrm{~ms}$ to $538 \pm 275 \mathrm{~ms}$, $\left.\mathrm{F}_{1}=-3.3, p=0.01\right)$ and $R R\left(40 \pm 15\right.$ to $\left.75 \pm 19, \mathrm{~F}_{1}=3.5, p<0.01\right)$ were greater in females than males in vagotomized animals without chest compression (E duration: 1245 $\pm 444 \mathrm{~ms}$ to $538 \pm 275 \mathrm{~ms}, \mathrm{~F}_{1}=-3.3, p=0.01 ; \mathrm{RR}: 40 \pm 15$ to $\left.75 \pm 19, \mathrm{~F}_{1}=3.5, p<0.01\right)$, and with the addition of chest compression (E duration: $837 \pm 220 \mathrm{~ms}$ to $368 \pm 180 \mathrm{~ms}, \mathrm{~F}_{1}$ $=-4.0, p<0.01 ; \mathrm{RR}: 52 \pm 11$ to $\left.81 \pm 12, \mathrm{~F}_{1}=4.6, p<0.01\right)$.

\section{Poincaré plots of breathing phase durations}

Figure 4-3 displays Poincaré plots of the E duration variability over 30 second periods for the different modulations of afferent feedback. The points are tightly clustered for post-vagotomy males, indicating low variability, while the more dispersed points for the vagotomized females illustrate the opposite effect. When lidocaine was nebulized, there was no change in the tightness of the clustering, but the entire cluster shifted to the left (indicating a decrease in E duration) in males; in females the cluster shifted to the 
right. When spinal feedback was reduced with pleural lidocaine injections, there was a slight decrease in variability in males and females, as indicated by more clustered points.

\section{$\underline{\text { Variability }}$}

For all animals, control TRC was compared to weight, and the resulting $\mathrm{R}^{2}$ value of 0.41 (Figure $4-4 a$ ) indicates that $40 \%$ of the variance is correlated with the weight of the animal. In figure 4-4b, weight of each animal is plotted against sex, and shows that the female group had the largest and smallest weights as well as the largest variance in weights.

\section{Discussion}

The goal of this study was to evaluate breathing during a mechanical challenge, while systematically altering spinal and vagal afferent feedback. Mechanical challenge was achieved by restricting chest wall movements (via banding), along with performing vagotomy, selectively anesthetizing PSRs, or reducing spinal feedback. The present results demonstrate that: a) the classic respiratory response to vagotomy is not solely due to eliminating PSR feedback, b) there is a balance in vagal and spinal feedback that alters diaphragm activity in the early expiration ("yield" Figure 4-1) phase, and c) injection of lidocaine into the pleural space modulates breathing, most likely by inhibiting spinal afferent feedback.

\section{Chest compression}

External pressure to the thoracic cavity was first applied by Adrian (Adrian, 1933), who reported an increase in RR and vagal feedback. The RR effects have been replicated in humans and dogs (Bland, Lazerou, Dyck, \& Cherniack, 1967), cats and dogs (Shannon, 1975), and rabbits (D'Angelo, Miserocchi, \& Agostoni, 1976). As we 
hypothesized, chest compression increased RR, but the increase was only significant in male animals. Of note, increased RR is often a result of hypercapnia (respiratory acidosis due to insufficient alveolar ventilation). Though we did not directly measure blood gases during this study, other work indicate that the increases in RR due to chest compression are not a direct result of increased chemical drive in either intact or vagotomized animals (Shannon, 1979b) (in contrast to an elastic load (Shannon, 1975)). Shannon attributed the alteration in RR to stimulation of chest wall mechanoreceptors (Shannon, 1975).

Yield: a novel description of diaphragm activity during early expiration

The classic definition of yield is to "give way". In the present study, we derive the term from its use in locomotion, specifically referencing active contraction of leg extensors during weight acceptance (Hildebrand, 1959). More specifically, knee and ankle extensor contraction during flexion provides a buffer/cushion from impact forces and prevents destabilization (Hildebrand, 1959). Goslow and colleagues (Goslow Jr, Reinking, \& Stuart, 1973) definitively showed that yield-related muscle recruitment must be an active contractile element to be effective. Additionally, they demonstrated that as rate of locomotion increases, muscle recruitment during yield also increases in amplitude and duration.

During breathing diaphragm, parasternal and external intercostals activity steadily increases in amplitude to reach a maximum peak producing inspiratory airflow; this activity comprises the "I" phase (Figure 4-2) (Pitts, Poliacek, Rose, Reed, Condrey, Tsai, Zhou, Davenport, \& Bolser, 2018; Pitts, Rose, Poliacek, Condrey, Davenport, \& Bolser, 2015)). The I phase is immediately followed by the beginning of the E phase (termed E1 or post-I in the literature (Bautista, Sun, \& Pilowsky, 2014; Dutschmann, Jones, 
Subramanian, Stanic, \& Bautista, 2014)), which starts with a decrementing diaphragm burst and is then followed by a period of diaphragm quiescence. Although the early phase of exhalation has traditionally been described as passive (i.e. no active abdominal contraction) there is indeed muscle activity both from the diaphragm and expiratory laryngeal muscles. Rather than forcing air out of the lungs, these muscles activate to serve as a mechanical brake increasing laryngeal resistance and slowing exhalation (reviewed by Richter and Smith 2014 (Richter \& Smith, 2014)). This regulation of expiratory airflow is also important in conditions where groups of various inspiratory, expiratory, pharyngeal, and laryngeal muscles must be precisely controlled. This tight regulation is required during swallow, vocalization and cough; more specifically where the lungs act as bellows to store air for the expulsion that is required for those motor behaviors. This phase has been variously called "E1", "post-I", "early-E", and "E-dec"; here we are using the term "yield" to specifically refer to the cushioning properties (from remnant diaphragm, parasternal and external intercostal activity) of this event.

It has recently been proposed that the post-inspiratory complex (PiCo) in the brainstem functions as a network oscillator to coordinate this phase of breathing with other central respiratory oscillators, and to produce state-dependent modulations as required for metabolic demands or precision motor acts (Anderson, Garcia, Baertsch, Pollak, Bloom, Wei, Rai, \& Ramirez, 2016; Baertsch, Severs, Anderson, \& Ramirez, 2019). This is different and potentially adjunctive to laryngeal adduction, which has been classically used to define E1 (Bartlett Jr, 1989; Bartlett Jr, Remmers, \& Gautier, 1973; Harding, 1984). Interestingly, in the current experimental preparation the thyroarytenoid was active across the entire expiratory phase duration (Figure 4-1 and 4-2). This 
phenomenon of prolonged laryngeal adduction has also been demonstrated when breathing is at a mechanical disadvantage: infants, rodents (due to high lung compliance) (Dutschmann, Jones, Subramanian, Stanic, \& Bautista, 2014), laparotomy (Mondal, AbuHasan, Saha, Pitts, Rose, Bolser, \& Davenport, 2016), anesthetic states (Insalaco, Kuna, Costanza, Catania, Cibella, \& Bellia, 1991) and in cats anesthetized with chloraloseurethane (Sherrey \& Megirian, 1974) (but not with sodium pentobarbital (Sherrey \& Megirian, 1974)).

Our results also demonstrate that during application of a mechanical challenge (chest compression), yield duration in control conditions decreased, yield duration postvagotomy increased, and there was no change in yield following pleural injection of lidocaine. We hypothesize that removal of vagal feedback results in disinhibition, increasing yield duration. These results suggest that the yield phase is spinally mediated and that vagal feedback tonically inhibits this component of breathing. Remmers (Remmers, 1970) also concluded that supra-spinal inhibition and some spinal mechanism accounted for the response to chest compression.

This hypothesis has also been made about respiratory control in general. Gautier (Gautier, 1973) showed that removal of vagal feedback in one group of animals slowed RR due to prolonged I and E duration. Removal of spinal feedback via dorsal rhizotomy in another group of animals increased RR due to shortened I and E. When both vagal and spinal feedback were removed by bilateral vagotomy and dorsal rhizotomy, respectively, respiratory parameters did not significantly differ from those of intact animals (Gautier, 1973), suggesting that breathing is properly maintained by mechanisms balancing vagal and spinal feedback. 


\section{Laryngeal contribution to breathing (E1)}

Interaction between thyroarytenoid (laryngeal adductor) activity and respiratory pattern is modulated by vagally mediated volume feedback onto adductor motoneurons (Insalaco, Kuna, Costanza, Catania, Cibella, \& Bellia, 1991). Additionally, Bolser and Remmers (Bolser \& Remmers, 1989) showed that stimulation of intercostal (thoracic) afferents depolarized expiratory vagal motoneurons, presumed to be laryngeal adductor motoneurons. Thyroarytenoid activity also increased in response to stimulation of intercostal mechanoreceptors (Bolser \& Remmers, 1989). When these receptors were stimulated during inspiration, thyroarytenoid motoneurons were activated; during expiration they were augmented (Shannon, Bolser, \& Lindsey, 1987). In the current study, application of chest compression resulted in early excitation of laryngeal adduction during yield and altered muscle pattern activity throughout the expiratory phase (Figure 4-1). The activation of the thyroarytenoid muscles across the entire expiratory period limits further speculation about regulation of phase duration.

\section{$\underline{\text { Spinal afferent feedback }}$}

Lidocaine infused into the pleural space locally anesthetizes non-myelinated fibers of the peritoneum and the pleural space (Duron \& Marlot, 1980), as well as superficial mechano- and sensory receptors of the diaphragm, but has no effect on intercostal Golgi tendon organs and muscle spindles in the intercoastal muscles. Bolser et al. (1988) showed that muscle/rib vibration inhibits inspiratory-related phrenic activity. This "inspiratory inhibitory reflex" is a result of activating intercostal Golgi tendon organs, rather than the muscle spindle endings that had been previously described (Bolser, Lindsey, \& Shannon, 1987). Furthermore, thoracic dorsal rhizotomies (T1-T12) 
have effects on RR and lung volume, which are believed to be caused by the loss of chest wall proprioception feedback onto medullary respiratory neurons (Gautier, 1973;

Shannon, 1977; Shannon, 1986). During loading by tracheal occlusion, mechanoreceptors with afferent fibers in the cervical $\mathrm{C} 3-\mathrm{C} 7$ and thoracic $\mathrm{T} 1-\mathrm{T} 9$ regions are responsible for changes in medullary respiratory activity (Shannon, Shear, Mercak, Bolser, \& Lindsey, 1985). During chest compression, $R R$ increases, and this response is eliminated by thoracic dorsal rhizotomy (Shannon, 1979a). Collectively, this evidence indicates that characteristics of breathing can be strongly modulated by afferent spinal feedback.

Ramos (GARCIA, 1959), Campbell (Campbell \& Howell, 1962), Eccles, Sears and Shealy (Eccles, Sears, \& Shealy, 1962) and their colleagues all suggested that thoracic cavity proprioceptive feedback significantly contributes to breathing patterns (Campbell \& Howell, 1962). Three years later, Von Euler (von Euler \& Peretti, 1966) proposed that intercostal muscle spindles act as a "follow up length servo" by continuously adjusting muscle tension in response to volume demand. Using chest compression as a respiratory stimulant, the present study manipulated vagal and spinal feedback. Our results demonstrate that removal of vagal feedback in the presence of chest compression greatly influenced the yield phase of expiration (Figure 4-1), and that removing pleura-related spinal feedback during chest compression influenced inspiration, expiration, and RR in females but not males. While pleural lidocaine administration produced some changes, at the low lung volumes produced by chest compression these changes may not be primary effectors of breathing pattern. 


\section{$\underline{\text { Vagal feedback and sex as a biological variable }}$}

It has long been known that the vagus nerve contains PSR afferents (Kubin, Alheid, Zuperku, \& McCrimmon, 2006; Larrabee \& Knowlton, 1946; Schelegle \& Green, 2001), and that eliminating PSR feedback alters the breathing response to rapid lung inflation (Adrian, 1933; Gautier, 1973; Hammouda \& Wilson, 1932). Studies by Cross et al. (1976) in human and dog and by Fahim et al. (1979) in cat demonstrated that aerosolized bupivacaine blocked the majority of PSRs, diminishing the Hering-Breuer inflation reflex. This, along with the lack of an inflation response in the present data, leads us to conclude that nebulization of $10 \%$ lidocaine reduced/eliminated PSR feedback. In the present experimental conditions, alteration of PSR-specific activity was not a significant contributor to the effects produced by chest wall constriction. However, vagotomy did produce a significant, albeit sex-specific, response.

In the present study, during control conditions, females had longer total cycle durations (TRC) and reduced respiratory rates (RR) compared to males, due to an increase in expiratory duration (E). This sex difference was enhanced with vagotomy, with only females demonstrating E duration prolongation, while both sexes had similar trends in I duration increases. There is limited information about sex differences in respiratory control, and many papers that present mixed animal groups do not specifically examine sex differences.

The sex difference we observed during chest compression may be due to thoracic geometry, chest wall compliance, or restriction band size relative to chest size. Alveoli of female rats are larger in quantity and smaller in size than those of males (Massaro, Mortola, \& Massaro, 1995), resulting in larger alveolar surface area to body mass ratios 
in females compared to males (Carey, Card, Voltz, Arbes, Germolec, Korach, \& Zeldin, 2007; Massaro, Mortola, \& Massaro, 1995). Males have a larger lung volume but a smaller volume to body mass ratio than females (Carey, Card, Voltz, Arbes, Germolec, Korach, \& Zeldin, 2007; Massaro \& Massaro, 2006). Females have a smaller rib cage and a shorter diaphragm than males of the same height, and inspiratory intercostal muscles make a greater contribution to breathing in females compared to males (Bellemare, Jeanneret, \& Couture, 2003). Due to the inclination or angle of female ribs, the ribcage can accommodate greater volume expansion and increasing intercostal force compared to males (Bellemare, Jeanneret, \& Couture, 2003; LoMauro \& Aliverti, 2018). The result of this is that female breathing involves more thoracic contribution to movement, while male breathing involves more diaphragm contribution to movement (Bellemare, Jeanneret, \& Couture, 2003; Hutchinson, 1846; LoMauro \& Aliverti, 2018). We do not know if these anatomical differences are also present in the rat, but they do provide insight into possible causes for our observed sex differences during chest compression and afferent feedback manipulations.

\section{$\underline{\text { Limitations }}$}

Female animals had a $243 \%$ increase in variability compared to males, as indicated by the standard deviation values throughout this study. This may be caused in part by the fact that the smallest and largest animals in this study were female (Figure 44), or by variations in estrus cycle. It may be important in future work to compare effects across estrus cycles or use ovariohysterectomized animals. Additionally, the number of female rats in our nebulized cohort was small due to animal death from cardio-respiratory failure. The nebulized lidocaine protocol was performed on 8 females, only 3 of which 
survived. The weight range of the animals that did not survive was $0.275 \mathrm{~kg}$ to $0.430 \mathrm{~kg}$. The weight range of the animals that did survive was $0.34 \mathrm{~kg}$ to $0.55 \mathrm{~kg}$. Anesthesia also has potential confounding effects. Known effects of sodium pentobarbital on gamma motoneurons could have reduced muscle spindle proprioceptive feedback in our animals. However, similar effects are reported in vagotomy studies using different anesthetics such as chloralose (Adrian, 1933) and dial-urethane (Shannon, Zechman, \& Frazier, 1972), and Adrian (Adrian, 1933) concluded that anesthesia or decerebration had little impact on PSR activity.

\section{Conclusion}

We propose that considering the E1 phase of breathing as a respiratory yield state could help in interpreting differences in mechanistic descriptions of "late-I" activity versus "early-E" activity. We hypothesize that respiratory yield could be strongly regulated by spinally-mediated proprioceptive afferent feedback. This has potential implications for spinal cord injuries with thoracic level involvements, especially for the patients' ability to produce robust responses to state-dependent respiratory challenges via local spinal circuits. 


\section{Table 4-1.}

Means, standard deviation (SD), $p$-values and direction of change for breathing parameters during control and chest compression conditions are shown below for both $\underline{\text { male and female. }}$

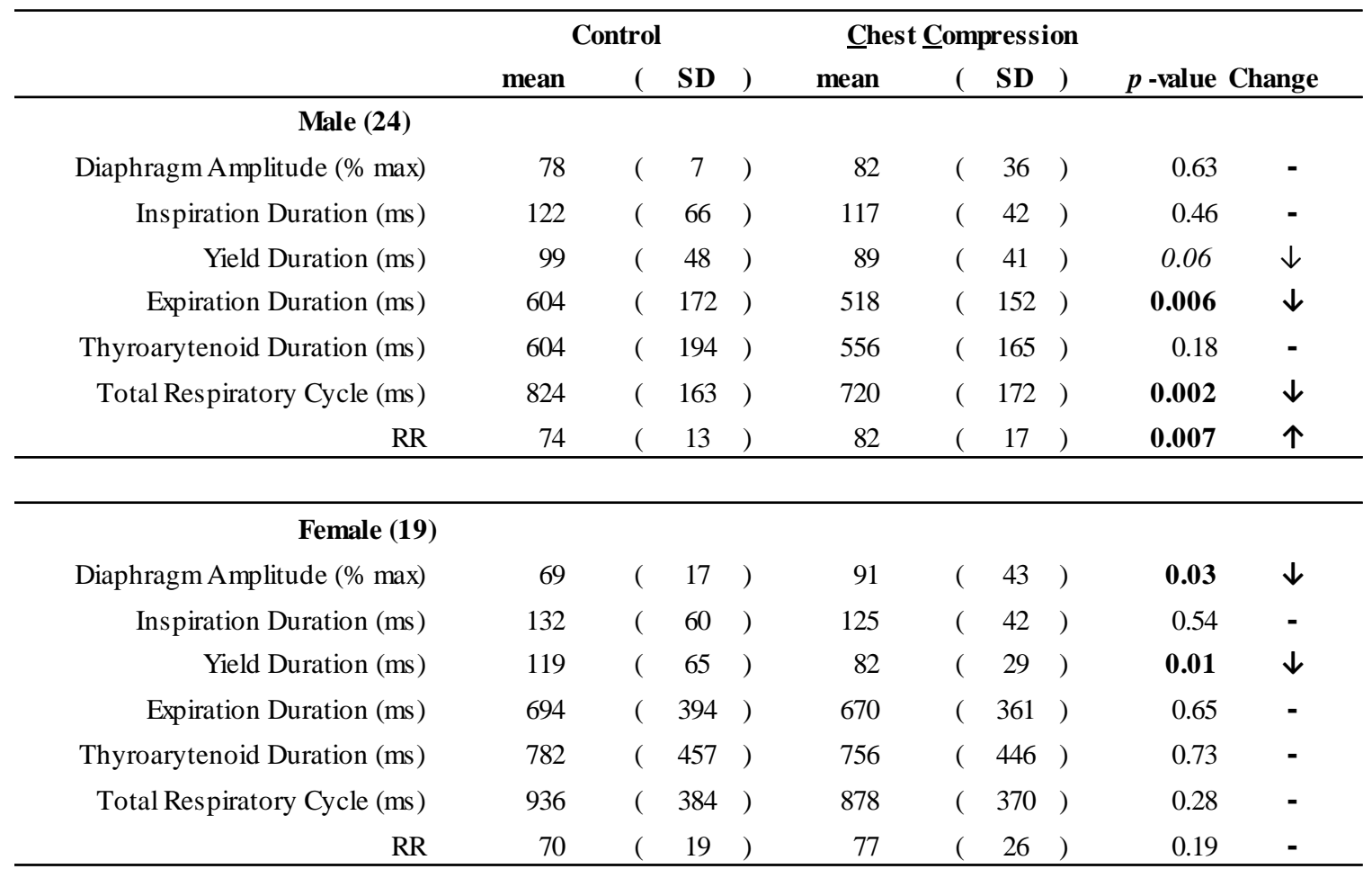

Diaphragm amplitude is normalized to maximum of control and shown as a percentage.

Respiratory rate (RR) was calculated as number of cycles within a 30 second period multiplied by 2 . Reported $p$-values are from Students paired t-test. Significance is bolded at values $\leq 0.05$ and approach to significance at values $0.05>\mathrm{x} \leq 0.07$ is italicized. 
Table 4-2.

Means, standard deviation (SD), p-values and direction of change for breathing parameters during control and feedback modulation conditions are shown below for both male and female.

\begin{tabular}{|c|c|c|c|c|c|c|c|c|c|c|}
\hline \multirow{2}{*}{$\mathbf{A}$} & \multicolumn{3}{|c|}{ Control } & \multicolumn{6}{|c|}{ Vagotomy } & \multirow[b]{2}{*}{ Change } \\
\hline & mean & ( & SD & ) & mean & ( & SD & ) & $p$-value & \\
\hline \multicolumn{11}{|l|}{ Male (6) } \\
\hline Diaphragm Amplitude (\% max) & 80 & ( & 4 & ) & 117 & ( & 53 & ) & 0.13 & - \\
\hline Inspiration Duration (ms) & 134 & ( & 37 & ) & 184 & ( & 38 & ) & 0.06 & $\uparrow$ \\
\hline Yield Duration (ms) & 111 & ( & 28 & ) & 132 & ( & 37 & ) & 0.14 & - \\
\hline Expiration Duration (ms) & 622 & ( & 152 & ) & 538 & ( & 275 & ) & 0.49 & - \\
\hline Thyroarytenoid Duration (ms) & - & ( & - & ) & - & ( & - & ) & - & - \\
\hline Total Respiratory Cycle (ms) & 837 & ( & 141 & ) & 850 & ( & 300 & ) & 0.91 & - \\
\hline $\mathrm{RR}$ & 73 & ( & 12 & ) & 75 & ( & 19 & ) & 0.74 & - \\
\hline \multicolumn{11}{|l|}{ Female (6) } \\
\hline Diaphragm Amplitude (\% max) & 72 & ( & 15 & ) & 129 & ( & 78 & ) & 0.11 & - \\
\hline Inspiration Duration (ms) & 124 & ( & 72 & ) & 274 & ( & 100 & ) & 0.07 & $\uparrow$ \\
\hline Yield Duration (ms) & 99 & ( & 29 & ) & 162 & ( & 92 & ) & 0.13 & - \\
\hline Expiration Duration (ms) & 849 & ( & 408 & ) & 1245 & ( & 444 & ) & 0.02 & $\uparrow$ \\
\hline Thyroarytenoid Duration (ms) & - & ( & - & ) & - & ( & - & ) & - & - \\
\hline Total Respiratory Cycle (ms) & 1050 & ( & 357 & ) & 1647 & ( & 489 & ) & 0.01 & $\uparrow$ \\
\hline $\mathrm{RR}$ & 62 & ( & 22 & ) & 40 & ( & 15 & ) & 0.04 & $\downarrow$ \\
\hline
\end{tabular}




\begin{tabular}{|c|c|c|c|c|c|c|c|c|c|c|}
\hline \multirow{2}{*}{$\mathbf{B}$} & \multicolumn{3}{|c|}{ Control } & \multicolumn{6}{|c|}{ Nebulize } & \multirow[b]{2}{*}{ Change } \\
\hline & mean & ( & SD & ) & mean & ( & SD & ) & $p$-value & \\
\hline \multicolumn{11}{|l|}{ Male (6) } \\
\hline Diaphragm Amplitude (\% max) & 76 & ( & 12 & ) & 108 & ( & 40 & ) & 0.09 & - \\
\hline Inspiration Duration (ms) & 88 & ( & 13 & ) & 226 & ( & 291 & ) & 0.32 & - \\
\hline Yield Duration(ms) & 79 & ( & 17 & ) & 81 & ( & 27 & ) & 0.91 & - \\
\hline Expiration Duration (ms) & 663 & ( & 129 & ) & 530 & ( & 241 & ) & 0.20 & - \\
\hline Thyroarytenoid Duration (ms) & 619 & ( & 156 & ) & 539 & ( & 260 & ) & 0.70 & - \\
\hline Total Respiratory Cycle (ms) & 824 & ( & 131 & ) & 831 & ( & 375 & ) & 0.97 & - \\
\hline RR & 74 & ( & 10 & ) & 89 & ( & 52 & ) & 0.54 & - \\
\hline \multicolumn{11}{|l|}{ Female (3) } \\
\hline Diaphragm Amplitude (\% max) & 74 & ( & 14 & ) & 91 & ( & 40 & ) & 0.40 & - \\
\hline Inspiration Duration (ms) & 130 & ( & 48 & ) & 130 & ( & 7 & ) & 0.99 & - \\
\hline Yield Duration (ms) & 79 & ( & 34 & ) & 79 & ( & 21 & ) & 0.93 & - \\
\hline Expiration Duration (ms) & 639 & ( & 169 & ) & 733 & ( & 165 & ) & 0.61 & - \\
\hline Thyroarytenoid Duration (ms) & - & ( & - & ) & - & ( & - & ) & - & - \\
\hline Total Respiratory Cycle (ms) & 839 & ( & 123 & ) & 942 & ( & 150 & ) & 0.51 & - \\
\hline RR & 70 & ( & 12 & ) & 63 & ( & 10 & ) & 0.60 & - \\
\hline & \multicolumn{3}{|c|}{ Control } & \multicolumn{7}{|c|}{ Pleural Injection } \\
\hline & mean & ( & SD & ) & mean & ( & SD & ) & $p$-value & Change \\
\hline \multicolumn{11}{|l|}{ Male (8) } \\
\hline Diaphragm Amplitude (\% max) & 78 & ( & 8 & ) & 73 & ( & 36 & ) & 0.73 & - \\
\hline Inspiration Duration (ms) & 147 & ( & 104 & ) & 169 & ( & 78 & ) & 0.58 & - \\
\hline Yield Duration (ms) & 123 & ( & 71 & ) & 126 & ( & 26 & ) & 0.88 & - \\
\hline Expiration Duration (ms) & 614 & ( & 251 & ) & 468 & ( & 171 & ) & 0.03 & $\downarrow$ \\
\hline Thyroarytenoid Duration (ms) & 642 & ( & 221 & ) & 508 & ( & 36 & ) & 0.30 & - \\
\hline Total Respiratory Cycle (ms) & 880 & ( & 220 & ) & 758 & ( & 129 & ) & 0.09 & - \\
\hline RR & 71 & ( & 17 & ) & 81 & ( & 13 & ) & 0.10 & - \\
\hline \multicolumn{11}{|l|}{ Female (6) } \\
\hline Diaphragm Amplitude (\% max) & 64 & ( & 25 & ) & 110 & ( & 51 & ) & 0.10 & - \\
\hline Inspiration Duration (ms) & 142 & ( & 49 & ) & 237 & ( & 134 & ) & 0.06 & $\uparrow$ \\
\hline Yield Duration (ms) & 160 & ( & 60 & ) & 133 & ( & 72 & ) & 0.48 & - \\
\hline Expiration Duration (ms) & 713 & ( & 572 & ) & 533 & ( & 427 & ) & 0.14 & - \\
\hline Thyroarytenoid Duration (ms) & 874 & ( & 759 & ) & 845 & ( & 503 & ) & 0.90 & - \\
\hline Total Respiratory Cycle (ms) & 1004 & ( & 579 & ) & 902 & ( & 429 & ) & 0.29 & - \\
\hline $\mathrm{RR}$ & 72 & ( & 25 & ) & 75 & ( & 24 & ) & 0.52 & - \\
\hline
\end{tabular}

Diaphragm amplitude is normalized to maximum of control and shown as a percentage.

Respiratory rate (RR) was calculated as number of cycles within a 30 second period

multiplied by 2. Reported $p$-values are from Students paired t-test. Significance is bolded 
at values $\leq 0.05$ and approach to significance at values $0.05>\mathrm{x} \leq 0.07$ is italicized. Table A shows data for male and female under control and post vagotomy conditions, B under nebulized conditions and $\mathrm{C}$ post pleural injection. 
Table 4-3.

Means, standard deviation (SD), p-values and direction of change for breathing

parameters during chest compression and feedback modulation conditions are shown

below for both male and female.

\begin{tabular}{|c|c|c|c|c|c|c|c|c|c|c|}
\hline \multirow{2}{*}{$\mathbf{A}$} & \multicolumn{4}{|c|}{ Chest $\underline{\text { Compression }}$} & \multicolumn{5}{|c|}{ CC+Vagotomy } & \multirow[b]{2}{*}{ Change } \\
\hline & mean & ( & SD & ) & mean & ( & SD & ) & $p$-value & \\
\hline \multicolumn{11}{|l|}{ Male (6) } \\
\hline Diaphragm Amplitude (\% max) & 69 & ( & 22 & ) & 97 & ( & 54 & ) & 0.16 & - \\
\hline Inspiration Duration (ms) & 117 & ( & 14 & ) & 211 & ( & 106 & ) & 0.08 & - \\
\hline Yield Duration (ms) & 93 & ( & 16 & ) & 147 & ( & 31 & ) & 0.002 & $\uparrow$ \\
\hline Expiration Duration (ms) & 454 & ( & 167 & ) & 368 & ( & 180 & ) & 0.10 & - \\
\hline Thyroarytenoid Duration (ms) & - & ( & - & ) & - & ( & - & ) & - & - \\
\hline Total Respiratory Cycle (ms) & 652 & ( & 196 & ) & 695 & ( & 204 & ) & 0.30 & - \\
\hline $\mathrm{RR}$ & 86 & ( & 15 & ) & 81 & ( & 12 & ) & 0.37 & - \\
\hline \multicolumn{11}{|l|}{ Female (6) } \\
\hline Diaphragm Amplitude (\% max) & 78 & ( & 37 & ) & 171 & ( & 119 & ) & 0.11 & - \\
\hline Inspiration Duration (ms) & 107 & ( & 15 & ) & 221 & ( & 70 & ) & 0.02 & $\uparrow$ \\
\hline Yield Duration (ms) & 78 & ( & 14 & ) & 154 & ( & 50 & ) & 0.02 & $\uparrow$ \\
\hline Expiration Duration (ms) & 864 & ( & 319 & ) & 837 & ( & 220 & ) & 0.89 & - \\
\hline Thyroarytenoid Duration (ms) & - & ( & - & ) & - & ( & - & ) & - & - \\
\hline Total Respiratory Cycle (ms) & 1047 & ( & 325 & ) & 1185 & ( & 283 & ) & 0.51 & - \\
\hline RR & 61 & ( & 17 & ) & 52 & ( & 11 & ) & 0.33 & - \\
\hline \multirow{2}{*}{$\mathbf{B}$} & \multicolumn{4}{|c|}{ Chest Compression } & \multicolumn{5}{|c|}{ CC+Nebulize } & \\
\hline & mean & ( & SD & ) & mean & ( & SD & ) & $p$-value & Change \\
\hline \multicolumn{11}{|l|}{ Male (6) } \\
\hline Diaphragm Amplitude (\% max) & 78 & ( & 44 & ) & 117 & ( & 58 & ) & 0.01 & $\uparrow$ \\
\hline Inspiration Duration (ms) & 85 & ( & 22 & ) & 111 & ( & 31 & ) & 0.21 & - \\
\hline Yield Duration (ms) & 73 & ( & 17 & ) & 85 & ( & 36 & ) & 0.55 & - \\
\hline Expiration Duration (ms) & 509 & ( & 97 & ) & 588 & ( & 222 & ) & 0.50 & - \\
\hline Thyroarytenoid Duration (ms) & 476 & ( & 266 & ) & 586 & ( & 195 & ) & 0.60 & - \\
\hline Total Respiratory Cycle (ms) & 661 & ( & 104 & ) & 782 & ( & 241 & ) & 0.38 & - \\
\hline RR & 85 & ( & 25 & ) & 74 & ( & 18 & ) & 0.46 & - \\
\hline \multicolumn{11}{|l|}{ Female (3) } \\
\hline Diaphragm Amplitude (\% max) & 117 & ( & 15 & ) & 137 & ( & 70 & ) & 0.71 & - \\
\hline Inspiration Duration (ms) & 148 & ( & 44 & ) & 141 & ( & 22 & ) & 0.75 & - \\
\hline Yield Duration (ms) & 62 & ( & 11 & ) & 87 & ( & 38 & ) & 0.28 & - \\
\hline Expiration Duration (ms) & 442 & ( & 234 & ) & 499 & ( & 187 & ) & 0.78 & - \\
\hline Thyroarytenoid Duration (ms) & - & ( & - & ) & - & ( & - & ) & - & - \\
\hline Total Respiratory Cycle (ms) & 652 & ( & 209 & ) & 727 & ( & 202 & ) & 0.72 & - \\
\hline RR & 99 & ( & 41 & ) & 89 & ( & 29 & ) & 0.80 & - \\
\hline
\end{tabular}




\begin{tabular}{|c|c|c|c|c|c|c|c|c|c|c|}
\hline$\gamma$ & \multicolumn{4}{|c|}{ Chest Compression } & \multicolumn{5}{|c|}{ CC+Pleural Injection } & \multirow[b]{2}{*}{ Change } \\
\hline & mean & ( & SD & ) & mean & ( & SD & ) & $p$-value & \\
\hline \multicolumn{11}{|l|}{ Male (8) } \\
\hline Diaphragm Amplitude (\% max) & 99 & ( & 40 & ) & 94 & ( & 44 & ) & 0.47 & - \\
\hline Inspiration Duration (ms) & 146 & ( & 57 & ) & 150 & ( & 60 & ) & 0.84 & - \\
\hline Yield Duration (ms) & 105 & ( & 64 & ) & 125 & ( & 87 & ) & 0.08 & - \\
\hline Expiration Duration (ms) & 597 & ( & 188 & ) & 545 & ( & 205 & ) & 0.31 & - \\
\hline Thyroarytenoid Duration (ms) & 644 & ( & 114 & ) & 598 & ( & 88 & ) & 0.30 & - \\
\hline Total Respiratory Cycle (ms) & 848 & ( & 183 & ) & 820 & ( & 165 & ) & 0.52 & - \\
\hline $\mathrm{RR}$ & 73 & ( & 13 & ) & 76 & ( & 15 & ) & 0.39 & - \\
\hline \multicolumn{11}{|l|}{ Female (6) } \\
\hline Diaphragm Amplitude (\% max) & 97 & ( & 62 & ) & 182 & ( & 111 & ) & 0.05 & $\uparrow$ \\
\hline Inspiration Duration (ms) & 124 & ( & 30 & ) & 186 & ( & 53 & ) & 0.02 & $\uparrow$ \\
\hline Yield Duration (ms) & 98 & ( & 43 & ) & 126 & ( & 93 & ) & 0.31 & - \\
\hline Expiration Duration (ms) & 638 & ( & 457 & ) & 353 & ( & 277 & ) & 0.03 & $\downarrow$ \\
\hline Thyroarytenoid Duration (ms) & 765 & ( & 698 & ) & 605 & ( & 390 & ) & 0.40 & - \\
\hline Total Respiratory Cycle (ms) & 857 & ( & 511 & ) & 651 & ( & 294 & ) & 0.10 & - \\
\hline RR & 81 & ( & 25 & ) & 103 & ( & 32 & ) & 0.03 & $\uparrow$ \\
\hline
\end{tabular}

Diaphragm amplitude is normalized to maximum of control and shown as a percentage.

Respiratory rate (RR) was calculated as number of cycles within a 30 second period

multiplied by 2. Reported $p$-values are from Students paired t-test. Significance is bolded at values $\leq 0.05$ and approach to significance at values $0.05>\mathrm{x} \leq 0.07$ is italicized. Table A shows data for male and female when chest compression was performed under control and post vagotomy conditions, $\mathrm{B}$ under nebulized conditions and $\mathrm{C}$ post pleural injection. 
Figure 4-1. Thyroarytenoid activity with chest compression.
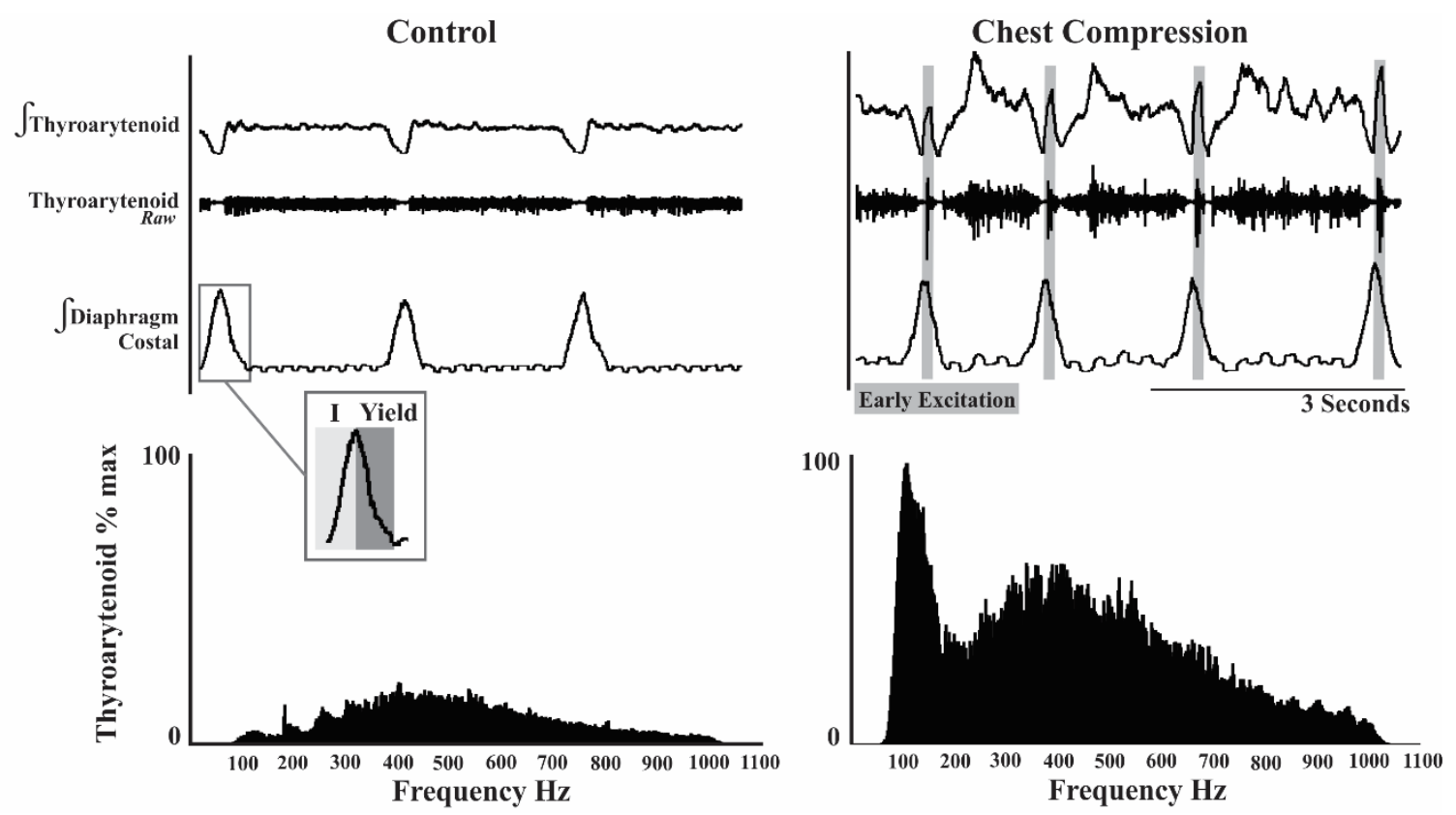

(Top) power spectrum analysis of thyroarytenoid muscle activity under control and chest compression conditions across 30 seconds of eupnea. (Bottom) EMG traces of corresponding muscle activity showing the activity change in the muscle pattern when chest compression is applied. The grey vertical rectangles represent the early activation of thyroarytenoid during yield phase of breathing. 
Figure 4-2. Diagram of proposed breathing phase.

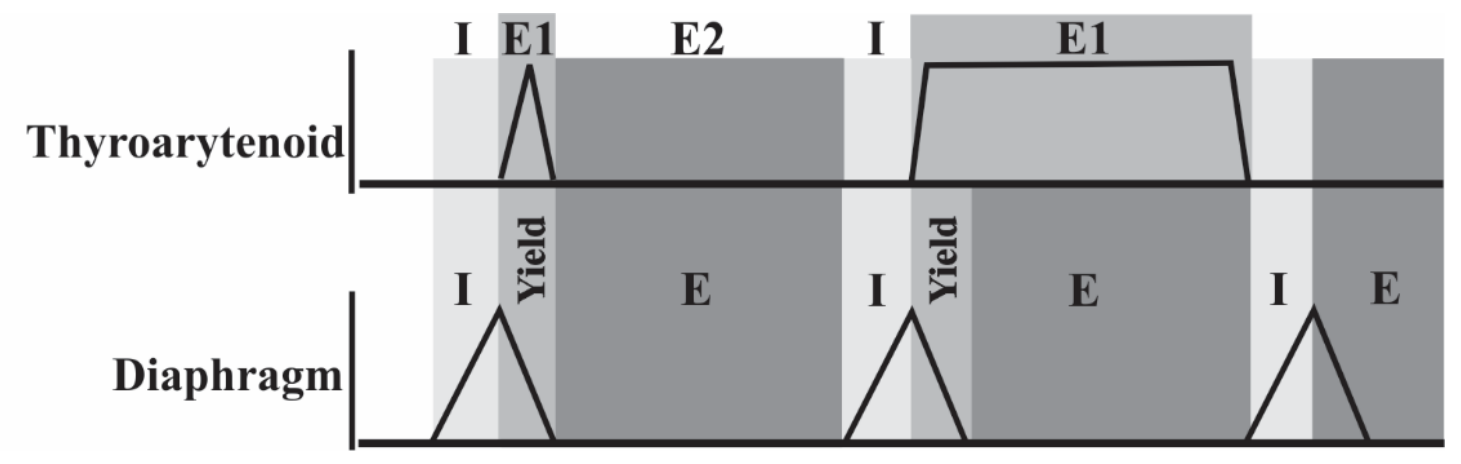

When looking at breathing via the diaphragm there are three phases: inspiration (I) (onset of diaphragm activity to peak diaphragm activity), yield (peak diaphragm activity to offset diaphragm activity) and expiration (E) (offset diaphragm activity to next onset diaphragm activity). When looking at breathing with the addition of the thyroarytenoid (vocal fold adductor) activity expiration is divided into multiple sub phases. The activity of the thyroarytenoid muscle during the yield phase is termed early or active expiration (E1). The period during the offset of the thyroarytenoid until the onset of the next diaphragm activation is given the term late or passive expiration (E2). E1 is active control of the thyroarytenoid muscle during expiration and E2 is the passive movement of lung recoil. In the case of this study there was no E2 phase only E1, activation of the thyroarytenoid muscle at the peak activation of the diaphragm and inactivation of thyroarytenoid at the onset of the next diaphragm activation. 
Figure 4-3. Poincaré plots demonstrating difference in expiration duration variability across all three afferent feedback interventions in both male and female.
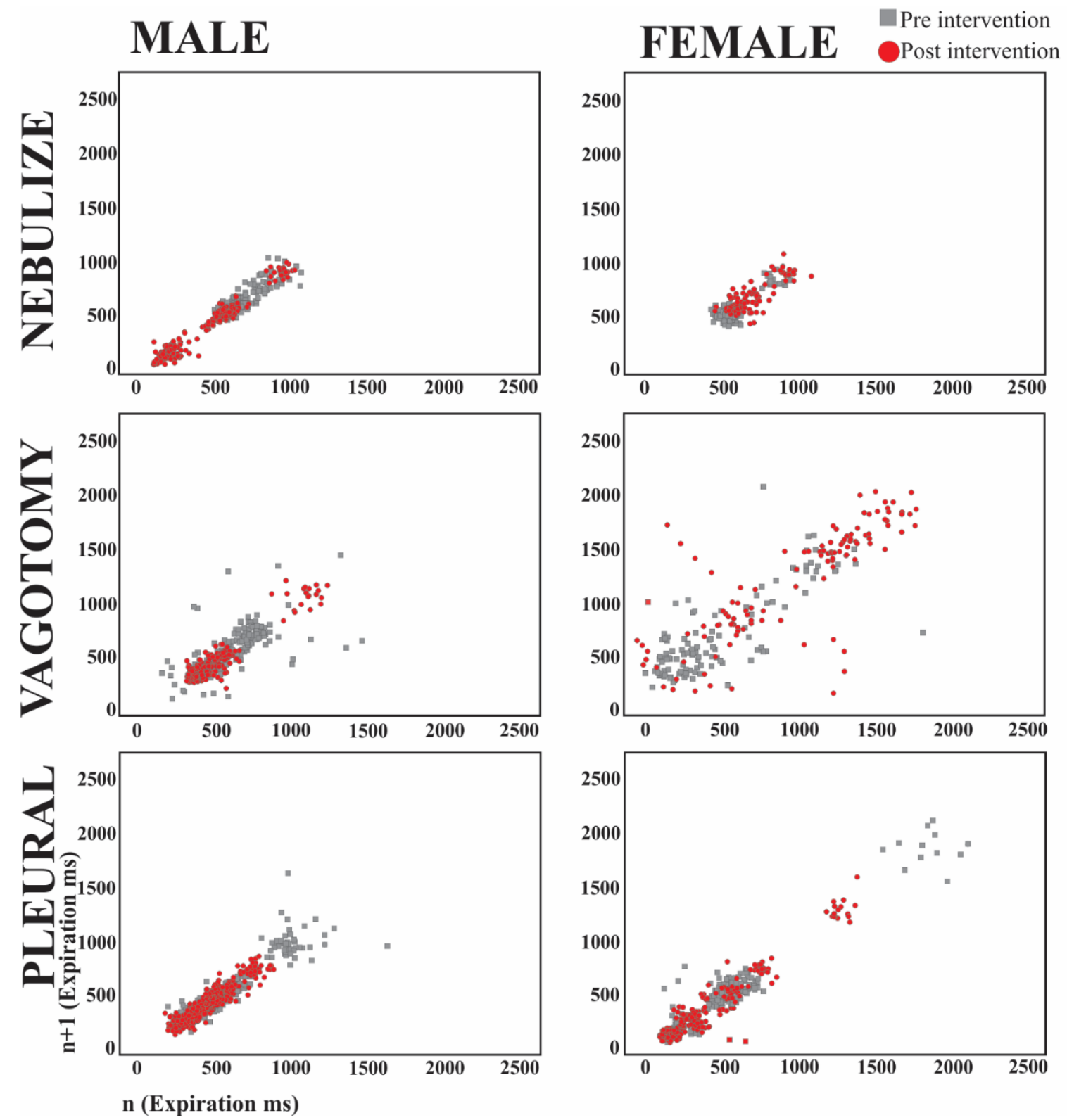

The grey squares represent control conditions and the red circles represent post intervention. Males after vagotomy and pleural injection show tighter clustering. 
Figure 4-4. Scatterplots of weight distribution.
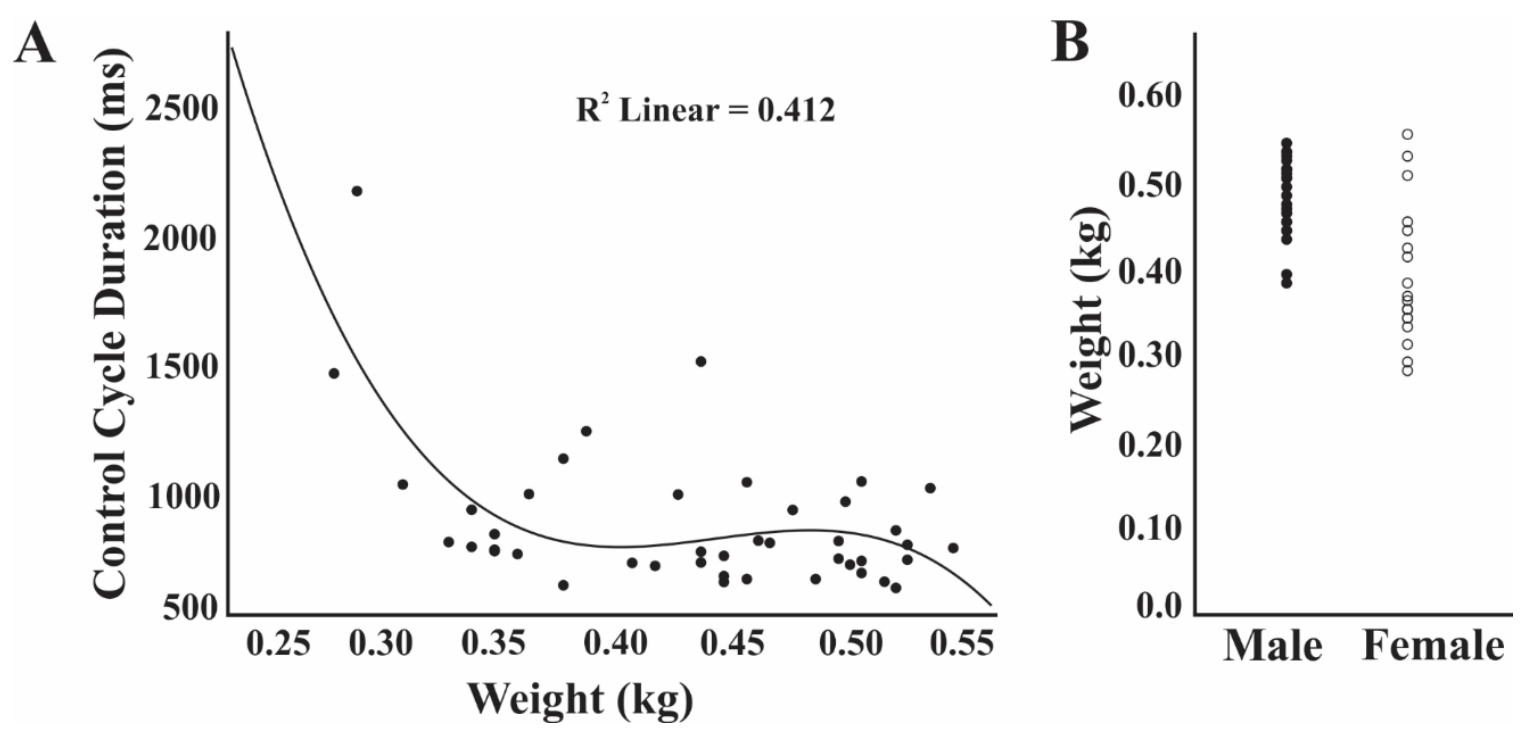

\begin{abstract}
A) Scatter plots showing distribution of weight versus cycle duration and B) weight distribution of male versus female. These scatter plots show the large variability of female rat weights versus male.
\end{abstract}




\section{CHAPTER 5}

\section{SEX SPECIFIC VAGAL AND SPINAL MODULATION OF SWALLOW AND ITS COORDINATION WITH BREATHING (PART 2)}

\section{Introduction}

The purpose of airway protection is to coordinate the passage of air into and out of the lungs and foreign material into the esophagus. In 1789, Patten (1789) described the first case of dysphagia (disorder of swallow), and in 1816 Magendie (1816) described the three phases of swallow: oral, pharyngeal and esophageal. Kronecker and Meltzer in 1880 (1880) discovered that swallow required the integration of brainstem respiratory centers with the activity of six cranial nerves (Kronecker \& Meltzer, 1882), and they described swallow as the most complex "all or none" reflex. In 1887 Marckwald (1888) and Wassilieff (1887) identified a "swallow center" located in the $4^{\text {th }}$ ventricle of the brainstem of the rabbit and described the influence of swallow on breathing. In 1915, Miller and Sherrington (1915) concluded that stimulation of many different medullary locations can elicit swallow. The possibility of spinal influences on swallow was supported by Sumi in 1963 (1963a), who reported that groups of medullary and spinal inspiratory and expiratory neurons were either excited or inhibited by swallow, even when the animals were paralyzed and artificially ventilated.

These seminal studies form a foundation for the swallow field, and since then swallow has been studied in vivo in the mouse (Sang \& Goyal, 2001), rat (Kessler \& 
Jean, 1985; Ouahchi, Letelier, Bon-Mardion, Marie, Tardif, \& Verin, 2011), bat (Greet \& De Vree, 1984), cat (Dick, Oku, Romaniuk, \& Cherniack, 1993b; Gestreau, Milano, Bianchi, \& Grélot, 1996; Harada, Takakusaki, Kita, Matsuda, Nonaka, \& Sakamoto, 2005; Horton, Segers, Nuding, O’Connor, Alencar, Davenport, Lindsey, Morris, \& Gestreau, 2018; Pitts, Rose, Mortensen, Poliacek, Sapienza, Lindsey, Morris, Davenport, \& Bolser, 2013b; Spearman, Poliacek, Rose, Bolser, \& Pitts, 2014; Suzuki, Nakazawa, \& Shiba, 2010), rabbit (McFarland \& Lund, 1993; Uchida, Yamada, \& Sato, 1994), pig (Thexton, Crompton, \& German, 2007), sheep (Jean, 1984b), goat (Feroah, Forster, Fuentes, Lang, Beste, Martino, Pan, \& Rice, 2002), monkey (Chiao, Larson, Yajima, Ko, \& Kahrilas, 1994; McNamara Jr \& Moyers, 1973), and human (Huff, Day, English, Reed, Zouboules, Saran, Leacy, Mann, Peltonen, \& O'Halloran, 2018; Huff, Reed, Smith, Brown, Ovechkin, \& Pitts, 2018b; McFarland, Martin-Harris, Fortin, Humphries, Hill, \& Armeson, 2016; Wheeler Hegland, Huber, Pitts, Davenport, \& Sapienza, 2011b; Wheeler Hegland, Huber, Pitts, \& Sapienza, 2009b). Swallow has also been studied in situ (Bautista \& Dutschmann, 2014; Gestreau, Grélot, \& Bianchi, 2000; Gestreau, Milano, Bianchi, \& Grélot, 1996; Hashimoto, Sugiyama, Fuse, Umezaki, Oku, Dutschmann, \& Hirano, 2019) and in vitro (Kogo, Yamanishi, Koizumi, \& Matsuya, 2002) and modeled in silico (Bolser, Gestreau, Morris, Davenport, \& Pitts, 2013b; Pitts, Morris, Lindsey, Davenport, Poliacek, \& Bolser, 2012b). In humans, swallow has been most studied in populations with diseases including Parkinson's disease (Hegland, Okun, \& Troche, 2014; Pitts, Bolser, Rosenbek, Troche, \& Sapienza, 2008; Pitts, Troche, Mann, Rosenbek, Okun, \& Sapienza, 2010; Troche, Okun, Rosenbek, Musson, Fernandez, Rodriguez, Romrell, Pitts, Wheeler-Hegland, \& Sapienza, 2010; Troche, Schumann, Brandimore, 
Okun, \& Hegland, 2016), stroke (Hammond, Goldstein, Horner, Ying, Gray, GonzalezRothi, \& Bolser, 2009; Smith Hammond, Goldstein, Zajac, Gray, Davenport, \& Bolser, 2001), and head and neck cancer (Brodsky, McFarland, Dozier, Blair, Ayers, Michel, Gillespie, Day, \& Martin-Harris, 2010).

Despite the progress that has been made in the last century to understand the complex behavior of swallow, our mechanistic understanding of this important behavior is limited. Classically, swallow has been regarded as a strictly brainstem-mediated behavior, but more recent studies have determined that afferent feedback is important in the coordination of swallow with breathing cycle (Huff, Reed, Smith, Brown, Ovechkin, \& Pitts, 2018b; McFarland, Martin-Harris, Fortin, Humphries, Hill, \& Armeson, 2016; Wheeler Hegland, Huber, Pitts, Davenport, \& Sapienza, 2011b; Wheeler Hegland, Huber, Pitts, \& Sapienza, 2009b). In the cat, swallow normally occurs in the late expiratory (E2) phase of the cough breathing cycle (Pitts, Rose, Mortensen, Poliacek, Sapienza, Lindsey, Morris, Davenport, \& Bolser, 2013b), but upper abdominal laparotomy produces a significant shift of swallow to the inspiratory phase of the breathing cycle. Several studies in the human demonstrate that — regardless if swallow occurs as a single (Wheeler Hegland, Huber, Pitts, \& Sapienza, 2009b) or sequential events (Wheeler Hegland, Huber, Pitts, Davenport, \& Sapienza, 2011b), with a thin or thick consistency bolus (McFarland, Martin-Harris, Fortin, Humphries, Hill, \& Armeson, 2016; Wheeler Hegland, Huber, Pitts, \& Sapienza, 2009b), or if the system is challenged to coordinate with cough epochs (Huff, Reed, Smith, Brown, Ovechkin, \& Pitts, 2018b)—swallow occurs during a targeted lung volume of 45-65\% of vital capacity (Huff, Reed, Smith, Brown, Ovechkin, \& Pitts, 2018b; McFarland, Martin-Harris, Fortin, Humphries, Hill, \& 
Armeson, 2016; Wheeler Hegland, Huber, Pitts, Davenport, \& Sapienza, 2011b; Wheeler Hegland, Huber, Pitts, \& Sapienza, 2009b). In a previous publication, we reported this and developed the concept of lung volume targeting which can explain swallow occurrence across any phase of cough in the human (Huff, Reed, Smith, Brown, Ovechkin, \& Pitts, 2018b).

Lung volume regulation relies on both vagal and spinal afferent feedback, but the effects of these feedback sources on swallow occurrence is unknown. In this study, we selectively reduced different types of afferent feedback with three different manipulations: vagotomy to eliminate vagal feedback, lidocaine nebulization to suppress pulmonary stretch receptor (PSR) feedback, and lidocaine infusion into the pleural space to reduce spinal feedback from pleural afferents. Due to the strong evidence that PSR and other vagal feedback influences respiratory phase regulation, we hypothesized that loss of these important sensory feedback components would shift swallow occurrence more toward the inspiratory phase of the breathing cycle.

\section{Methods}

Experiments were performed on 43 anesthetized spontaneously breathing Sprague Dawley $(\mathrm{SpD})$ retired breeder rats $[24$ male $(0.49 \pm 0.04 \mathrm{~kg})$ and 19 female $(0.39 \pm$ 0.08kg)]. Protocol was approved by University of Louisville Institutional Animal Care and Use Committee (IACUC). The animals were initially anesthetized with gaseous isoflurane while a femoral intravenous (i.v.) cannula was placed for administration of sodium pentobarbital ( $25 \mathrm{mg} / \mathrm{kg}$, i.v.). Isoflurane was discontinued and supplementary doses of sodium pentobarbital were administered as needed throughout the experiment. Anesthetic level was evaluated by withdrawal reflex of the forelimb and hindlimb and 
licking in response to oral water administration. A dose of atropine sulfate $(0.01 \mathrm{mg} / \mathrm{kg}$, i.v.) was given at the beginning of the experiment to reduce secretions from repeated tracheal stimulation. Following administration of atropine sulfate, a tracheostomy was performed and followed by incision into the esophagus to place a 20 gauge catheter to measure esophageal pressure. Body temperature was maintained using a heating pad.

Electromyograms (EMG) were recorded using bipolar insulated fine wire electrodes according to the technique of Basmajinan and Stecko (Basmajian \& Stecko, 1962). Six muscles were used to evaluate swallow and/or breathing function: mylohyoid, geniohyoid, thyropharyngeus, bilateral placement of thyroarytenoid, and costal diaphragm. A small horizontal incision was made at the rostral end of the right digastric muscle exposing the surface of the mylohyoid and electrodes were placed in the right mylohyoid. A small horizontal incision was made on the rostral end of the left digastric continuing through to the left mylohyoid exposing the geniohyoid and electrodes were placed in the left geniohyoid. The thyroarytenoid electrodes were inserted through the cricothyroid window into the anterior portion of the vocal folds, which were visually inspected post-mortem. The thyropharyngeus muscles is a fan shaped muscles with the smallest portion attached to the thyroid cartilage; electrodes were placed at the rostral insertion of this muscle. For electrode placement of the costal diaphragm, palpation and elevation of the xyphoid process was followed by insertion of a needle directly caudal, and the needle was hooked underneath the xyphoid process near the costal diaphragm muscle attachment. Electrodes were placed bilaterally into the pectoralis muscle to record electrocardiogram (ECG) activity and to remove heart artifact from EMG traces. 


\section{$\underline{\text { Experimental Protocol }}$}

Three experimental protocols were performed on three cohorts of male and female SpD rats. A) An extra-thoracic vagotomy was performed in $12 \mathrm{SpD}$ rats [6 male $(0.48 \pm 0.03 \mathrm{~kg})$ and 6 female $(0.35 \pm 0.06 \mathrm{~kg})] . \mathrm{B})$ Lidocaine $(10 \%)$ was nebulized into the trachea in $13 \mathrm{SpD}$ rats [ 8 male $(2$ sham $)(0.40 \pm 0.03 \mathrm{~kg}), 5$ female $(2$ sham $)(0.39 \pm$ $0.09 \mathrm{~kg})] . \mathrm{C})$ Lidocaine (10\%) was injected into the pleural space in $18 \mathrm{SpD}$ rats [10 male (2 sham) $(0.46 \pm 0.05 \mathrm{~kg}), 8$ female $(2$ sham $)(0.41 \pm 0.08 \mathrm{~kg})]$.

\section{Removal/reduction of vagal feedback}

A) To remove all vagal afferent feedback, bilateral vagotomy at the level of the extra-thoracic trachea was performed on male and female rats in the supine position. The vagus nerves were dissected away from the sympathetic nerves and common carotid arteries. Silk suture (5-0) was looped around each vagus nerve with hemostat forceps clamped onto the suture ends for quick access after control trials had been completed. While lifting the suture attached to the hemostats, the vagus nerves were cut using spring scissors at the level of the $5^{\text {th }}-6^{\text {th }}$ tracheal ring. After bilateral vagotomy an inflation test was performed: $4 \mathrm{cc}$ of air was drawn into a $5 \mathrm{cc}$ syringe and quickly infused into the endotracheal tube to assure removal of PSR (lung volume) feedback. The order of the cuts were randomized (left vs right) across animals.

B) To selectively reduce vagal feedback from pulmonary stretch receptors, $10 \%$ lidocaine was nebulized into the trachea with the animal in the supine position. Using a compressor nebulizer (StrongHealth; particle size 0.5-5 $\mu \mathrm{m}$; average nebulization rate $0.2 \mathrm{~mL} / \mathrm{min}$ ), $10 \%$ Lidocaine (Cat No. L5647, 
Sigma-Aldrich) mixed with 2\% Evans Blue Dye (EBD, Cat No. E2129, Sigma-Aldrich) was nebulized for 15 minutes. Ten minutes after the completion of the nebulization, we performed an inflation test by injecting 4cc of air into the trachea. If the Hering-Breuer reflex was maintained (i.e. termination of inspiration followed by prolonged expiration), the animal then received an additional 5 minutes of nebulized lidocaine and was retested. This procedure was performed as necessary until the reflex was abolished. The addition of the dye allowed for post-mortem verification that the lidocaine penetrated the lung tissue and the intra- and extra-thoracic trachea. To minimize contamination of the lidocaine and dye into the air, a portable fume evacuation machine hovered over the mouthpiece of the nebulizer. To minimize contamination around the trachea, Vaseline-coated gauze was placed below and above the trachea, which covered any exposed area of the animal and blocked any potential absorption of lidocaine into the upper airway that was not specifically targeted by nebulized lidocaine..

\section{Reduction of spinal feedback}

C) To reduce spinal feedback, bilateral injections of $10 \%$ lidocaine mixed with $2 \%$ EBD were administered into the pleural space using methods from Mantilla et. al. (Mantilla, Zhan, \& Sieck, 2009). Animals were stabilized on their side while the rib cage was palpated to identify the fifth intercostal space, and the injection site was located and marked by a permanent marker, by measuring one inch rostral to the xyphoid process and moving laterally to the 
axial side of the rib. This was repeated on each axial side of the animal. At this location the skin was removed using skin scissors, and $20 \mu 1$ of lidocaine/EBD mixture was injected bilaterally using a 100- $\mu$ l Hamilton syringe with a 35 gauge beveled needle inserted $6 \mathrm{~mm}$. After both injections were complete, the animal was returned to supine position, and after a 5 minute waiting period an inflation test was performed to confirm that a reflex response was present, indicating that the lidocaine had not reached the PSRs or altered any other vagal afferent feedback.

In the companion paper to this study we state that lidocaine infused into the pleural space locally anesthetizes non-myelinated fibers of the peritoneum and the pleural space (Duron \& Marlot, 1980), as well as superficial mechano- and sensory receptors of the diaphragm, but has no effect on intercostal golgi tendon organs and muscle spindles.

Stimuli

Two stimuli were completed throughout various conditions in each protocol. Chest compression stimuli were performed during control conditions (before lidocaine or vagotomy interventions) and also after interventions. Swallow stimuli were performed during conditions with and without chest compression as well as post intervention conditions with and without chest compression.

Chest compression of the thoracic cavity was performed by placing a 2-inch thick Velcro band to restrict chest movement to target the of end-expiration of tidal volume. In order to monitor movement of the chest wall, a homemade piezoelectric chest strap made from a piezoelectric sensor inside a fire alarm and an elastic hair tie mounted on an 
aluminum plate was loosely strapped around the chest of the animal rostral to the Velcro restriction band. This piezoelectric chest strap allowed observation of the change in movement as a result of the restrictive band. Video was also taken for visual observation of the reduction in chest movement.

Swallow was induced by insertion of 1cc water into the oropharynx via a 1 inch long thin polyethylene catheter (diameter $2.37 \mathrm{~mm}$ ), attached to a 3cc syringe. Swallow was defined as a sequential activation of the mylohyoid, geniohyoid, thyroarytenoid muscles and costal diaphragm, if present, (representing the schluckatmung or swallow breath, Figure 5-1). Swallow stimuli were performed before and after intervention as well as during chest compression stimuli.

\section{$\underline{\text { Analysis }}$}

All EMG signals were amplified and filtered (100-1000 Hz). Signals were rectified and integrated (20ms) using Spike2 (Cambridge Electronic Design; Cambridge, England). EMG amplitude measures were normalized to the largest swallow in the control trial with and without chest compression. Swallow parameters measured: total swallow duration (onset of mylohyoid activation to offset of thyroarytenoid activation) and amplitude of mylohyoid, geniohyoid and thyroarytenoid. The inactivity of the thyroarytenoid in conjunction with mylohyoid and geniohyoid activity defines licking behaviors from swallow activity (Chiao, Larson, Yajima, Ko, \& Kahrilas, 1994). Without the activity of thyroarytenoid, the event was not included as a swallow. Swallow phase of breathing was marked accordingly: inspiration (I) was classified as the onset of the diaphragm activation to the peak of the diaphragm burst; "yield" was classified as the peak of the diaphragm burst to the offset of the diaphragm activation (Figure 5-1 and see 
below); expiration (E) was classified as the offset of diaphragm activation to the following diaphragm burst onset.

Yield is characterized by remnant diaphragm activity in early expiration that acts as a "cushion" to dampen forces from the chest wall onto the lungs. We derived this term from its use in locomotion studies, in which the term describes activation of knee and ankle extensor muscle to cushion the impact of forces on the body as the hips move over the knee (Hildebrand, 1959). The companion paper to this study presents a detailed description of this concept in respiration, and hypothesizes that characterizing early expiration as a yield event could aid in interpreting differences in late-I versus early-E activities of breathing.

Results are expressed as means \pm standard deviation (SD). Paired t-tests and Wilcoxon signed ranks tests were used as appropriate to statistically identify differences using SPSS statistical software (IBM Corporation). Analyses were made within groups (male and female) and between groups (male vs female). A difference was considered significant if the $p$-value was less than 0.05 .

\section{Results}

\section{$\underline{\text { Swallow with chest compression }}$}

Injection of water into the oropharynx elicited an average of $6 \pm 4$ swallows in males and $9 \pm 6$ swallows in females during control conditions; chest compression did not change swallow number (Table 5-1).

In control conditions, females produced 169 total swallows. Of those, $62 \%$ (104 of 169) occurred during E, 37\% (62 of 169) occurred during yield and 1\% (3 of 169) occurred during I. With application of chest compression, 135 swallows occurred, with 
$78 \%$ (105) occurring in E, 21\% (28) during yield and 1\% (2) during I. During chest compression there was a significant shift in swallow-breathing phase preference with more swallows occurring during $\mathrm{E}(\mathrm{z}=-3.2, p=0.001$; Table 5-2 and Figure 5-2b). Under control conditions, 154 swallows were elicited in males. Of those 66\% (101) occurred during E, $32 \%$ (49) in yield, and 2\% (4) in I; chest compression produced no significant change in swallow-breathing coordination (Table 5-2).

Compared to control conditions, chest compression increased mylohyoid EMG amplitude in males by $38 \%\left(\mathrm{t}_{22}=-2.6, p<0.05\right)$ and geniohyoid amplitude by $32 \%\left(\mathrm{t}_{22}=\right.$ $-2.3, p<0.05)$; but there were no significant changes in females (Table 5-1).

\section{Vagotomy}

Figure 5-1a and c show examples of the changes in swallow-related EMG activity following vagotomy (Table 5-3a). In males, mylohyoid EMG activity increased by 56\% and geniohyoid increased by $57 \%\left(\mathrm{t}_{3}=-11.1, p=0.002, \mathrm{t}_{3}=-9.4, p=0.003\right.$, respectively); in females, geniohyoid amplitude increased by $51 \%\left(\mathrm{t}_{4}=-2.4, p=0.07\right)$, but this increase was not significant. Bilateral extra-thoracic vagotomy produced no change in swallow number, duration, or swallow-breathing coordination (Table 5-4a).

When compared to chest compression alone, the addition of bilateral vagotomy (Table 5-5a) significantly decreased swallow number in males $\left(4 \pm 3\right.$ to $3 \pm 2, t_{5}=4.0, p$ $<0.010)$, and increased geniohyoid EMG activity $\left(\mathrm{t}_{4}=-3.2, p<0.049\right)$. In females, vagotomy produced a trend towards reduction in swallow number $(6 \pm 6$ to $3 \pm 4$, t5 = $2.4, \mathrm{p}<0.063)$. Vagotomy caused a significant change in swallow-breathing coordination in female animals only (Figure 5-2c), with $95 \%$ of swallows (18 of 19) occurring during $\mathrm{E}(\mathrm{z}=-2.5, p=0.011 ;$ Table 5-6a). 


\section{$\underline{\text { Local anesthesia of PSRs via lidocaine inhalation }}$}

Following local PSRs anesthesia males produced 19 swallows. Of those, 52\% (10 of 19) occurred during early expiratory yield, 47\% (9 of 19) occurred during E and none occurred during I. Compared to control there was a significant change in swallowbreathing coordination $(\mathrm{Z}=-1.89, p=0.59$; Table 5-4b) with more swallows occurring during yield

When compared to chest compression alone, the addition of lidocaine nebulization (Table 5-5b) significantly decreased swallow number in males from $(5 \pm 3$ to $2 \pm 2, \mathrm{t}_{5}=2.9, p=0.033$ ), but produced no change in females. This intervention produced no significant changes in swallow-breathing coordination (Table 5-6b). However, swallow duration was reduced in both male $\left(244 \pm 37 \mathrm{~ms}\right.$ to $198 \pm 41 \mathrm{~ms}, \mathrm{t}_{4}=$ 4.0, $p=0.014$ ) and female ( $342 \pm 60$ to $\left.217 \pm 45 \mathrm{~ms}, \mathrm{t}_{2}=8.5, p=0.014\right)$ groups, but there were no significant changes in EMG amplitudes of swallow-related muscles.

\section{$\underline{\text { Local anesthesia of pleural afferents via lidocaine injection }}$}

We locally anesthetized pleural afferents by injecting lidocaine into the pleural space (Table 5-3c). These injections caused a 30\% decrease in swallow-related mylohyoid amplitude $\left(\mathrm{t}_{7}=3.6, p=0.01\right)$ in males, and in females caused a $19 \%$ decrease in mylohyoid amplitude and a $25 \%$ decrease in geniohyoid amplitude $\left(\mathrm{t}_{4}=3.4, p=0.027\right.$, $\mathrm{t}_{4}=3.6, p=0.023$, respectively, Figure 5-1b and d).

When compared to chest compression alone (Table 5-5c), the addition of lidocaine injections produced a significant change in swallow-breathing coordination in female animals (Figure 5-2d), with $69 \%$ of swallows (20 of 29) occurring in E and 31\% (9 of 29) during yield: a significant shift to $\mathrm{E}(\mathrm{Z}=-2.65, p=0.008$, Table $5-6 \mathrm{c})$. In 
females, thyroarytenoid amplitude was reduced by $23 \%$, but this was non-significant $\left(\mathrm{t}_{4}=\right.$ $2.5, p=0.07)$.

\section{Discussion}

This is the first study to investigate the effects of both vagal and spinal afferent feedback on swallow-breathing coordination in the rat. Our results suggest that there are major sex differences in swallow-breathing coordination, and that disrupting vagal feedback produces different effects than disrupting spinal feedback. Male appear to rely more on PSR-mediated volume feedback, while alterations in spinal feedback produced greater effects in females. Our results confirm that both vagal and non-vagal afferent feedback sources are necessary for ensuring a stable swallow motor pattern in the rat.

\section{$\underline{\text { Sex differences in swallow-breathing coordination }}$}

Following PSR anesthesia in male animals, swallow-breathing coordination shifted toward swallow occurrence during yield (i.e. early expiration, defined by remnant diaphragm activity; Figure 5-1). For female animals, swallow occurrence shifted to late expiration when chest compression alone was applied, and also when vagal or spinal feedback was reduced (by vagotomy or pleural lidocaine injections) during chest compression.

In humans, swallow timing is dependent on lung volume (Huff, Reed, Smith, Brown, Ovechkin, \& Pitts, 2018b; McFarland, Martin-Harris, Fortin, Humphries, Hill, \& Armeson, 2016; Wheeler Hegland, Huber, Pitts, \& Sapienza, 2009b), which has been attributed to volume-related feedback via activation of PSRs. This is consistent with our results in the male rodents. Nebulization of lidocaine caused swallows to predominately occur during yield (early expiration), shifting the swallows closer to the inspiratory 
phase. Swallows that occur during the inspiratory phase are presumed to increase aspiration risk (Feroah, Forster, Fuentes, Lang, Beste, Martino, Pan, \& Rice, 2002; Martin-Harris, Brodsky, Price, Michel, \& Walters, 2003a), which we hypothesize may be due to a mechanical advantage of bolus movement from an area of high pressure (pharynx) to an area of low pressure (esophagus). The current results are consistent with our previous theory that lung volume is a major factor in swallow breathing phase preference (Huff, Reed, Smith, Brown, Ovechkin, \& Pitts, 2018b). The current results are also consistent with previous studies that demonstrated the majority of swallows occur during expiration (Pitts, Rose, Mortensen, Poliacek, Sapienza, Lindsey, Morris, Davenport, \& Bolser, 2013b). When volume feedback is reduced, or when transdiaphragmatic pressure is disrupted by laparotomy (Pitts, Rose, Poliacek, Condrey, Davenport, \& Bolser, 2015), swallow phase preference begins to move away from the classically predominant expiration phase and shifts toward the inspiration-to-expiration transition phase.

Considering that swallow-breathing coordination in females was altered only under chest compression conditions in the current study, we hypothesize that chest wall proprioception was the dominant feedback source in female rats. In addition to direct monitoring by PSRs, thoracic stretch receptors indirectly monitor lung volume (Lust, 2007) by detecting changes in muscle length and tension (Campbell \& Howell, 1962; Zechman Jr \& Wiley, 2011). During conditions of chest compression, swallows retained an expiratory preference, even when we altered vagal and spinal afferent feedback. In our companion study, chest compression prolonged expiration duration in female rats. The dominance of swallow during expiration could be attributed to the large proportion of the 
respiratory cycle that is spent in expiration, which would ensure adequate time for swallow to occur in safe conditions (Pitts, Rose, Mortensen, Poliacek, Sapienza, Lindsey, Morris, Davenport, \& Bolser, 2013b).

Female rats appear to rely more on the contribution of thoracic movements to breathing, in contrast to male animals, who appear to rely more on movement of the diaphragm (Bellemare, Jeanneret, \& Couture, 2003; Hutchinson, 1846; LoMauro \& Aliverti, 2018). Compared to males, females also have a smaller ratio of lung volume to body mass (Carey, Card, Voltz, Arbes, Germolec, Korach, \& Zeldin, 2007) and a smaller rib cage (Bellemare, Jeanneret, \& Couture, 2003). Considering that we used the same chest band for all experiments, it was not sized relative to the different chest wall sizes of male and female animals, suggests chest compression could have had a greater effect on females than males. Other physiological sex differences, such as hormones, could also influence swallow-breathing coordination.

Upper airway amplitude changes during swallow due to vagal and spinal feedback

When PSR activity is experimentally reduced, upper airway tone is increased in the cat and dog (van Lunteren \& Dick, 1989; van Lunteren, Haxhiu, \& Cherniack, 1989). When we reduced PSR activity by nebulizing lidocaine, swallow-related upper airway activity also increased, likely due to disinhibition (Bailey \& Fregosi, 2006). When we perturbed spinal feedback by injecting lidocaine into the pleural space, upper airway activity decreased, suggesting that spinal afferents provide excitatory modulation of upper airway activity during swallow. Together, these results indicate that mechanisms mediated by both vagal and spinal afferent feedback are important for the regulation of larger motor units during swallow (defined by alterations in EMG amplitude). 
Furthermore, since swallow amplitude was modulated when vagal or spinal feedback was perturbed, we propose that vagal/spinal afferent input balance is required for normal swallow behavior.

$\underline{\text { Swallow duration relies on both PSR and spinal feedback }}$

In conditions of chest compression, when PSR feedback was also reduced, swallow duration was decreased in both male and female animals. As volume feedback from both vagal and spinal sources appears to be important for swallow, experimentally and mechanically reducing PSR feedback, by nebulizing lidocaine during chest compression, would increase the risk of dysfunctional swallow. In this case, swallows may occur more quickly to maintain airway patency (Pitts, Rose, Mortensen, Poliacek, Sapienza, Lindsey, Morris, Davenport, \& Bolser, 2013b). The decrease in swallow duration that we observed could result from an underlying decrease in central swallow excitability, but this is unlikely, considering that swallow number and amplitude were unchanged.

\section{$\underline{\text { EMG amplitude and duration are not correlated }}$}

The results of this study further support our hypothesis that there are different central mechanisms for regulating swallow amplitude and duration. Clinically, it has been assumed that swallow duration positively correlates with force production, as defined by swallow phase relationships in videofluoroscopy exams (Spearman, Poliacek, Rose, Bolser, \& Pitts, 2014). We have now established that swallow-related EMG amplitude and duration are not correlated in cats (Pitts, Rose, Poliacek, Condrey, Davenport, \& Bolser, 2015; Reed, English, English, Huff, Poliacek, Musselwhite, Howland, Bolser, \& Pitts, 2019; Spearman, Poliacek, Rose, Bolser, \& Pitts, 2014), humans (Huff, Day, 
English, Reed, Zouboules, Saran, Leacy, Mann, Peltonen, O'Halloran, Sherpa, \& Pitts, 2018; Huff, Reed, Smith, Brown, Ovechkin, \& Pitts, 2018a), or rats (present study). The inability to assess this using visual metrics (videofluoroscopy and endoscopy) supports the need for development of "strength" related clinical metrics in order to better investigate this property of swallow pattern generation.

\section{$\underline{\text { Limitations }}$}

The data cohort of females in which lidocaine was nebulized is small, due in part to a high number of animal deaths from cardio-respiratory failure. This cohort originally consisted of 8 females, all of varying weights and estrus cycles, of which only 3 survived the protocol. Anesthesia also introduces potential limitations due to effects of sodium pentobarbital on gamma motoneurons. The dampening effects of this anesthetic may have reduced proprioceptive feedback in our study.

\section{Conclusion}

Our results provide evidence that, while the swallow central pattern generator is located in the brainstem, perturbations of peripheral feedback can disrupt swallow in predictable ways. This study adds to the body of evidence demonstrating that swallowbreathing coordination is dependent upon lung volume. This has potential clinical implications, as development of therapies targeting specific lung volumes to allow for safe swallowing would benefit patient populations for whom swallow is a risky behavior, such as patients with spinal cord injuries. 


\section{Table 5-1.}

Means, standard deviation (SD), $p$-values and direction of change for swallow parameters during control and chest compression conditions are listed for both male and female groups.

\begin{tabular}{|c|c|c|c|c|c|c|c|c|c|c|}
\hline & \multicolumn{4}{|c|}{ Control } & \multicolumn{4}{|c|}{ Chest Compression } & \multirow[b]{2}{*}{$p$-value } & \multirow[b]{2}{*}{ Change } \\
\hline & mean & ( & SD & ) & mean & ( & SD & ) & & \\
\hline \multicolumn{11}{|l|}{ Male (24) } \\
\hline Swallow Duration (ms) & 296 & ( & 73 & ) & 303 & ( & 77 & ) & 0.64 & - \\
\hline Swallow Number & 6 & ( & 4 & ) & 5 & ( & 4 & ) & 0.08 & - \\
\hline Mylohyoid Amplitude (\% max) & 72 & ( & 19 & ) & 111 & ( & 70 & ) & 0.02 & $\uparrow$ \\
\hline Geniohyoid Amplitude (\% max) & 78 & ( & 14 & ) & 110 & ( & 69 & ) & 0.03 & $\uparrow$ \\
\hline Thyroarytenoid Amplitude (\% max) & 87 & ( & 10 & ) & 115 & ( & 69 & ) & 0.06 & $\uparrow$ \\
\hline \multicolumn{11}{|l|}{ Female (19) } \\
\hline Swallow Duration (ms) & 301 & ( & 93 & ) & 290 & ( & 70 & ) & 0.41 & - \\
\hline Swallow Number & 9 & ( & 6 & ) & 7 & ( & 6 & ) & 0.17 & - \\
\hline Mylohyoid Amplitude (\% max) & 70 & ( & 19 & ) & 80 & ( & 41 & ) & 0.24 & - \\
\hline Geniohyoid Amplitude (\% max) & 76 & ( & 14 & ) & 85 & ( & 48 & ) & 0.39 & - \\
\hline Thyroarytenoid Amplitude (\% max) & 85 & ( & 10 & ) & 94 & ( & 21 & ) & 0.08 & - \\
\hline
\end{tabular}

Amplitude is normalized to maximum of control and shown as a percentage. Reported $p$ values are from Students paired t-test. Significance is bolded at $p$-values $\leq 0.05$ and $p$ values of $0.05>\mathrm{x} \leq 0.07$ are italicized. 
Table 5-2.

Number of swallows during each phase of breathing for control and chest compression conditions are listed for both male and female groups.

\begin{tabular}{rccccc}
\hline $\mathbf{A}$ & & $\begin{array}{c}\text { Control } \\
\text { \# of Swallows }\end{array}$ & $\begin{array}{c}\text { Vagotomy } \\
\text { \# of S wallows }\end{array}$ & $\boldsymbol{p}$-value & $\mathbf{Z}$ \\
\hline Male & & & 0.13 & -1.51 \\
Inspiration & 1 & 0 & & \\
Yield & 8 & 5 & & \\
Expiration & 16 & 19 & & \\
\hline Female & & & & -0.38 \\
Inspiration & 0 & 0 & & \\
Yield & 18 & 11 & & \\
Expiration & 26 & 17 & & \\
\hline
\end{tabular}

Reported $p$-values are from Wilcoxon signed ranks test. Significance is bolded at $p$ values $\leq 0.05$ and $p$-values of $0.05>\mathrm{x} \leq 0.07$ are italicized. 
Table 5-3.

Means, standard deviation (SD), $p$-values and direction of change for swallow parameters during control and feedback modulation conditions are listed for both male and female groups.

\begin{tabular}{|c|c|c|c|c|c|c|c|c|c|c|}
\hline & \multicolumn{4}{|c|}{ Control } & \multicolumn{5}{|c|}{ Vagotomy } & \multirow[b]{2}{*}{ Change } \\
\hline & mean & ( & SD & ) & mean & ( & SD & ) & $p$-value & \\
\hline \multicolumn{11}{|l|}{ Male (4) } \\
\hline Swallow Duration (ms) & 286 & ( & 49 & ) & 307 & ( & 84 & ) & 0.41 & - \\
\hline Swallow Number & 4 & ( & 2 & ) & 4 & ( & 3 & ) & 0.93 & - \\
\hline Mylohyoid Amplitude (\% max) & 79 & ( & 13 & ) & 135 & ( & 20 & ) & 0.002 & $\uparrow$ \\
\hline Geniohyoid Amplitude (\% max) & 86 & ( & 7 & ) & 143 & ( & 15 & ) & 0.003 & $\uparrow$ \\
\hline \multicolumn{11}{|l|}{ Female (5) } \\
\hline Swallow Duration (ms) & 300 & ( & 69 & ) & 311 & ( & 69 & ) & 0.72 & - \\
\hline Swallow Number & 7 & ( & 6 & ) & 5 & ( & 6 & ) & 0.34 & - \\
\hline Mylohyoid Amplitude (\% max) & 74 & ( & 18 & ) & 130 & ( & 101 & ) & 0.22 & - \\
\hline Geniohyoid Amplitude (\% max) & 70 & ( & 23 & ) & 121 & ( & 69 & ) & 0.07 & $\uparrow$ \\
\hline & \multicolumn{4}{|c|}{ Control } & \multicolumn{5}{|c|}{ Nebulize } & \\
\hline & mean & ( & SD & ) & mean & ( & SD & ) & $p$-value & Change \\
\hline \multicolumn{11}{|l|}{ Male (6) } \\
\hline Swallow Duration (ms) & 246 & ( & 52 & ) & 204 & ( & 41 & ) & 0.12 & - \\
\hline Swallow Number & 6 & ( & 2 & ) & 3 & ( & 3 & ) & 0.08 & - \\
\hline Mylohyoid Amplitude (\% max) & 61 & ( & 25 & ) & 88 & ( & 82 & ) & 0.44 & - \\
\hline Geniohyoid Amplitude (\% max) & 82 & ( & 10 & ) & 108 & ( & 24 & ) & 0.14 & - \\
\hline Thyroarytenoid Amplitude (\% max) & 86 & ( & 15 & ) & 70 & ( & 26 & ) & 0.28 & - \\
\hline \multicolumn{11}{|l|}{ Female (3) } \\
\hline Swallow Duration (ms) & 314 & ( & 57 & ) & 194 & ( & 58 & ) & 0.20 & - \\
\hline Swallow Number & 9 & ( & 10 & ) & 5 & ( & 6 & ) & 0.23 & - \\
\hline Mylohyoid Amplitude (\% max) & 76 & ( & 22 & ) & 48 & ( & 8 & ) & 0.10 & - \\
\hline Geniohyoid Amplitude (\% max) & 76 & ( & 23 & ) & 40 & ( & 18 & ) & 0.07 & $\downarrow$ \\
\hline Thyroarytenoid Amplitude (\% max) & 89 & ( & 13 & ) & 60 & ( & 46 & ) & 0.40 & - \\
\hline
\end{tabular}




\begin{tabular}{|c|c|c|c|c|c|c|c|c|c|c|}
\hline & \multicolumn{4}{|c|}{ Control } & \multicolumn{5}{|c|}{ Pleural Injection } & \multirow[b]{2}{*}{ Change } \\
\hline & mean & ( & SD & ) & mean & ( & SD & ) & $p$-value & \\
\hline \multicolumn{11}{|l|}{ Male (8) } \\
\hline Swallow Duration (ms) & 356 & ( & 69 & ) & 300 & ( & 112 & ) & 0.06 & $\downarrow$ \\
\hline Swallow Number & 9 & ( & 6 & ) & 6 & ( & 3 & ) & 0.15 & - \\
\hline Mylohyoid Amplitude (\% max) & 71 & ( & 16 & ) & 41 & ( & 23 & ) & 0.01 & $\downarrow$ \\
\hline Geniohyoid Amplitude (\% max) & 66 & ( & 19 & ) & 63 & ( & 48 & ) & 0.81 & - \\
\hline Thyroarytenoid Amplitude (\% max) & 85 & ( & 9 & ) & 78 & ( & 31 & ) & 0.56 & - \\
\hline \multicolumn{11}{|l|}{ Female (6) } \\
\hline Swallow Duration (ms) & 278 & ( & 55 & ) & 215 & ( & 37 & ) & 0.13 & - \\
\hline Swallow Number & 11 & ( & 7 & ) & 7 & ( & 8 & ) & 0.11 & - \\
\hline Mylohyoid Amplitude (\% max) & 60 & ( & 6 & ) & 41 & ( & 12 & ) & $\mathbf{0 . 0 3}$ & $\downarrow$ \\
\hline Geniohyoid Amplitude (\% max) & 69 & ( & 9 & ) & 44 & ( & 10 & ) & 0.02 & $\downarrow$ \\
\hline Thyroarytenoid Amplitude (\% max) & 82 & ( & 9 & ) & 67 & ( & 25 & ) & 0.23 & - \\
\hline
\end{tabular}

Amplitude is normalized to maximum of control and shown as a percentage. Reported $p$ values are from Students paired t-test. Significance is bolded at $p$-values $\leq 0.05$ and $p$ values of $0.05>\mathrm{x} \leq 0.07$ are italicized. Data is shown comparing control conditions to conditions of vagotomy (A), nebulized lidocaine (B), and pleural injection of lidocaine (C). 
Table 5-4.

Number of swallows during each phase of breathing during control and feedback $\underline{\text { modulation conditions are listed for both male and female groups. }}$

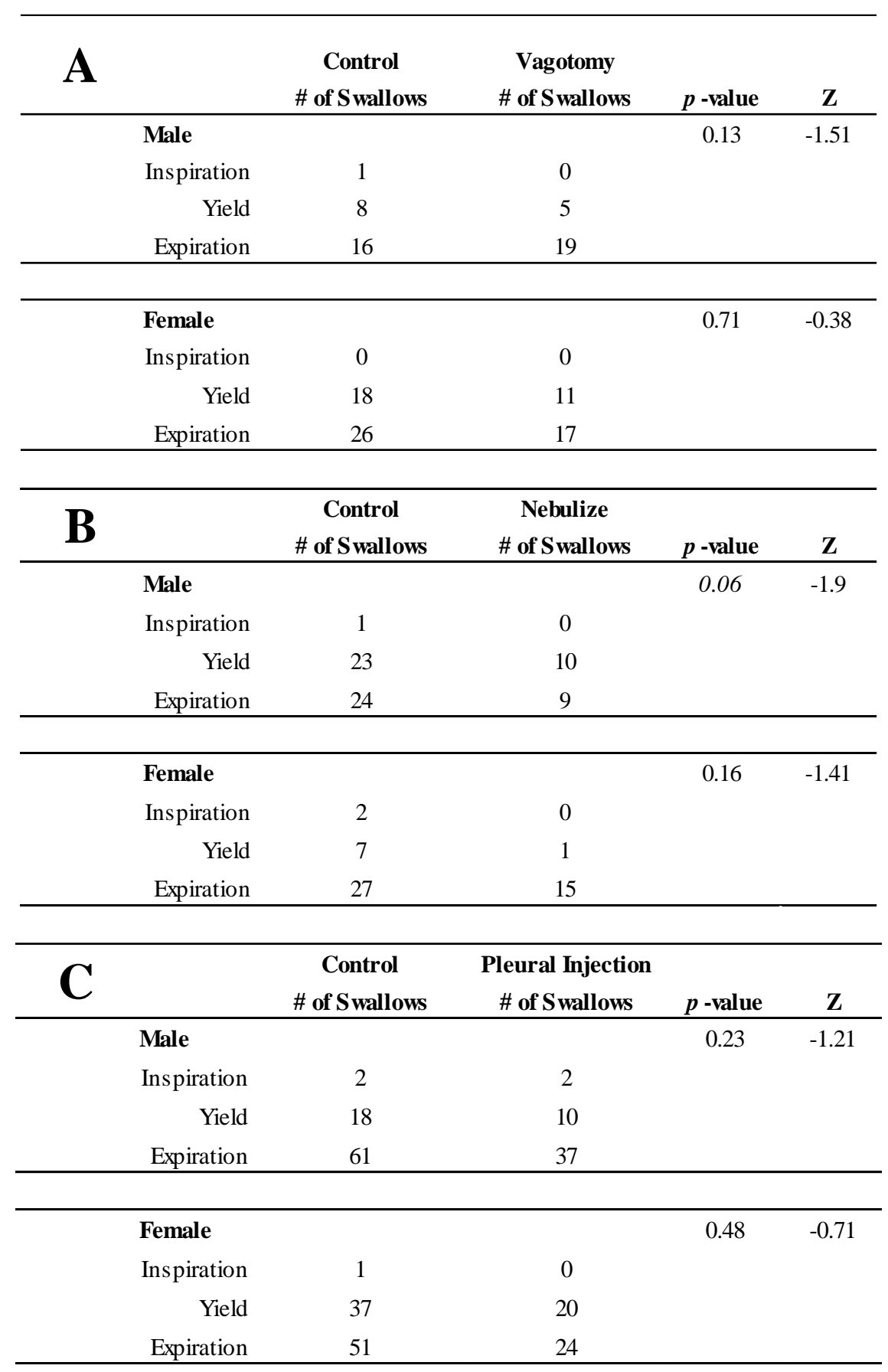


Reported $p$-values are from Wilcoxon signed ranks test. Significance is bolded at $p$ values $\leq 0.05$ and $p$-values of $0.05>\mathrm{x} \leq 0.07$ are italicized. Data is shown comparing control conditions to conditions of vagotomy (A), nebulized lidocaine (B), and pleural injection of lidocaine (C). 
Table 5-5.

Means, standard deviation (SD), $p$-values and direction of change for swallow parameters during chest compression and feedback modulation conditions are listed for both male and female groups.

\begin{tabular}{|c|c|c|c|c|c|c|c|c|c|c|}
\hline & \multicolumn{4}{|c|}{ Chest Compression } & \multicolumn{5}{|c|}{ CC+Vagotomy } & \multirow[b]{2}{*}{ Change } \\
\hline & mean & ( & SD & ) & mean & ( & SD & ) & $p$-value & \\
\hline \multicolumn{11}{|c|}{ Male (4) } \\
\hline Swallow Duration (ms) & 303 & ( & 69 & ) & 325 & ( & 82 & ) & 0.34 & - \\
\hline Swallow Number & 4 & ( & 3 & ) & 3 & ( & 2 & ) & 0.01 & $\downarrow$ \\
\hline Mylohyoid Amplitude (\% max) & 110 & ( & 11 & ) & 153 & ( & 35 & ) & 0.12 & - \\
\hline Geniohyoid Amplitude (\% max) & 110 & ( & 16 & ) & 160 & ( & 22 & ) & 0.05 & $\uparrow$ \\
\hline \multicolumn{11}{|c|}{ Female (4) } \\
\hline Swallow Duration (ms) & 280 & ( & 25 & ) & 322 & ( & 64 & ) & 0.29 & - \\
\hline Swallow Number & 6 & ( & 6 & ) & 3 & ( & 4 & ) & 0.06 & $\downarrow$ \\
\hline Mylohyoid Amplitude (\% max) & 84 & ( & 15 & ) & 243 & ( & 188 & ) & 0.19 & - \\
\hline Geniohyoid Amplitude (\% max) & 86 & ( & 15 & ) & 163 & ( & 80 & ) & 0.13 & - \\
\hline & \multicolumn{4}{|c|}{ Chest Compression } & \multicolumn{5}{|c|}{ CC+Nebulize } & \\
\hline & mean & ( & SD & ) & mean & ( & SD & ) & $p$-value & Change \\
\hline \multicolumn{11}{|c|}{ Male (5) } \\
\hline Swallow Duration (ms) & 244 & ( & 37 & ) & 198 & ( & 41 & ) & 0.02 & $\downarrow$ \\
\hline Swallow Number & 5 & ( & 3 & ) & 2 & ( & 2 & ) & 0.03 & $\downarrow$ \\
\hline Mylohyoid Amplitude (\% max) & 122 & ( & 54 & ) & 105 & ( & 78 & ) & 0.69 & - \\
\hline Geniohyoid Amplitude (\% max) & 102 & ( & 37 & ) & 119 & ( & 31 & ) & 0.19 & - \\
\hline Thyroarytenoid Amplitude (\% max) & 100 & ( & 49 & ) & 140 & ( & 161 & ) & 0.53 & - \\
\hline \multicolumn{11}{|c|}{ Female (3) } \\
\hline Swallow Duration (ms) & 342 & ( & 60 & ) & 217 & ( & 45 & ) & 0.01 & $\downarrow$ \\
\hline Swallow Number & 10 & ( & 6 & ) & 1 & ( & 0 & ) & 0.13 & - \\
\hline Mylohyoid Amplitude (\% max) & 82 & ( & 26 & ) & 54 & ( & 30 & ) & 0.12 & - \\
\hline Geniohyoid Amplitude (\% max) & 81 & ( & 22 & ) & 41 & ( & 28 & ) & 0.70 & $\downarrow$ \\
\hline Thyroarytenoid Amplitude (\% max) & 103 & ( & 19 & ) & 77 & ( & 63 & ) & 0.50 & - \\
\hline
\end{tabular}




\begin{tabular}{|c|c|c|c|c|c|c|c|c|c|c|}
\hline & \multicolumn{4}{|c|}{ Chest Compression } & \multicolumn{5}{|c|}{$\mathrm{CC}+$ Pleural Injection } & \multirow[b]{2}{*}{ Change } \\
\hline & mean & ( & SD & ) & mean & ( & SD & ) & $p$-value & \\
\hline \multicolumn{11}{|c|}{ Male (8) } \\
\hline Swallow Duration (ms) & 359 & ( & 68 & ) & 307 & ( & 78 & ) & 0.11 & - \\
\hline Swallow Number & 6 & ( & 5 & ) & 5 & ( & 3 & ) & 0.42 & - \\
\hline Mylohyoid Amplitude (\% max) & 76 & ( & 39 & ) & 64 & ( & 37 & ) & 0.51 & - \\
\hline Geniohyoid Amplitude (\% max) & 81 & ( & 23 & ) & 78 & ( & 52 & ) & 0.89 & - \\
\hline Thyroarytenoid Amplitude (\% max) & 100 & ( & 13 & ) & 86 & ( & 38 & ) & 0.26 & - \\
\hline \multicolumn{11}{|c|}{ Female (5) } \\
\hline Swallow Duration (ms) & 281 & ( & 42 & ) & 230 & ( & 47 & ) & 0.08 & - \\
\hline Swallow Number & 7 & ( & 8 & ) & 7 & ( & 7 & ) & 0.51 & - \\
\hline Mylohyoid Amplitude (\% max) & 60 & ( & 26 & ) & 45 & ( & 25 & ) & 0.34 & - \\
\hline Geniohyoid Amplitude (\% max) & 71 & ( & 26 & ) & 51 & ( & 27 & ) & 0.35 & - \\
\hline Thyroarytenoid Amplitude (\% max) & 98 & ( & 23 & ) & 75 & ( & 18 & ) & 0.07 & $\downarrow$ \\
\hline
\end{tabular}

Control chest compression (CC) is compared to CC plus intervention. Amplitude is normalized to maximum of control and shown as a percentage. Reported $p$-values are from Students paired t-test. Significance is bolded at $p$-values $\leq 0.05$ and $p$-values of $0.05>\mathrm{x} \leq 0.07$ are italicized. Data are shown comparing chest compression conditions to conditions adding vagotomy (A), nebulized lidocaine (B), and pleural injection of lidocaine (C). 


\section{Table 5-6.}

Number of swallows during each phase of during chest compression and feedback $\underline{\text { modulation conditions are listed for both male and female groups. }}$

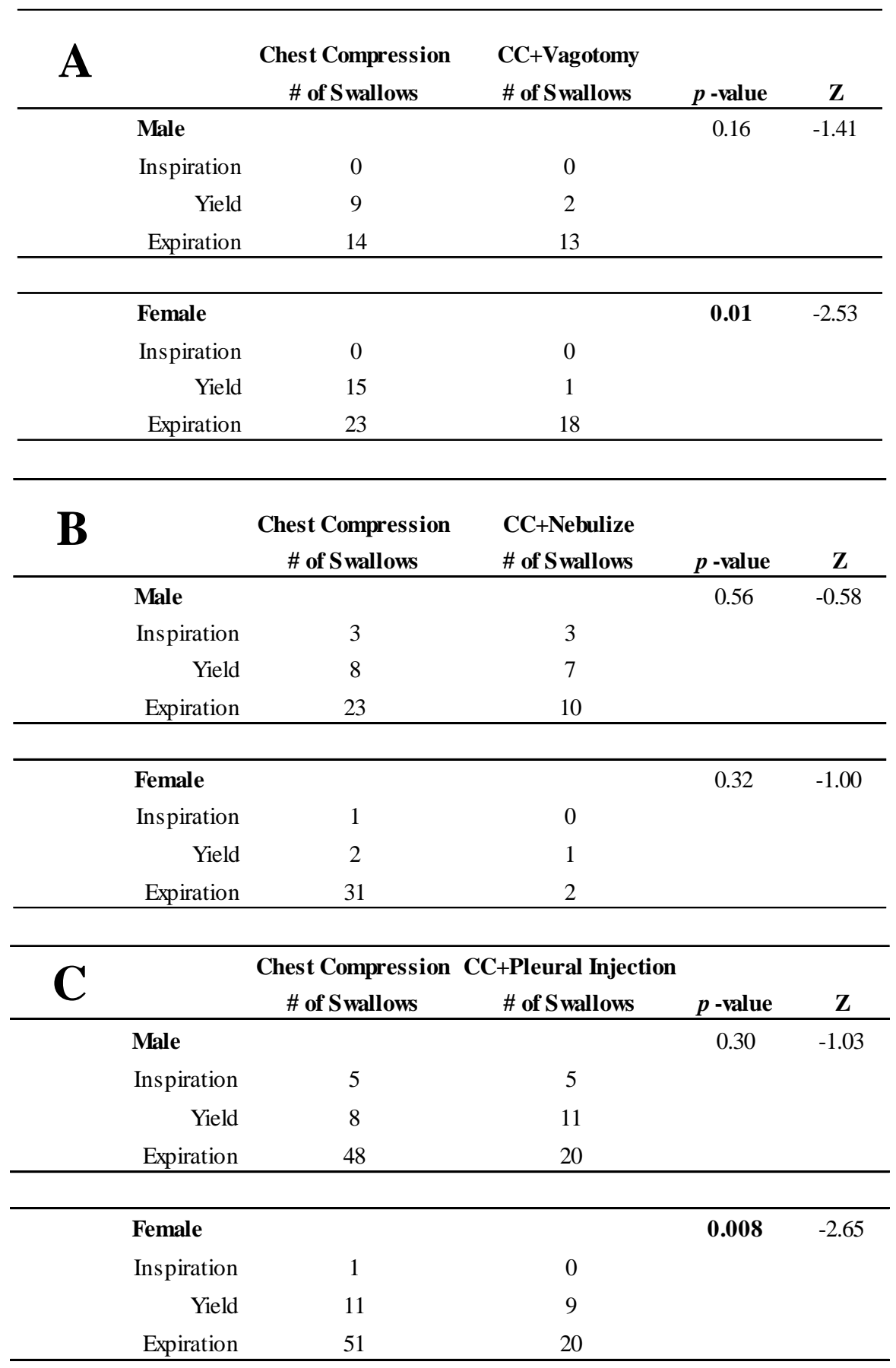


Control chest compression (CC) is compared to CC plus intervention. Reported $p$-values are from Wilcoxon signed ranks test. Significance is bolded at $p$-values $\leq 0.05$ and $p$ values of $0.05>\mathrm{x} \leq 0.07$ are italicized. Data are shown comparing control conditions to conditions of vagotomy (A), nebulized lidocaine (B), and pleural injection of lidocaine (C). 
Figure 5-1. Representative EMG traces of swallow activity before and after afferent feedback manipulations.

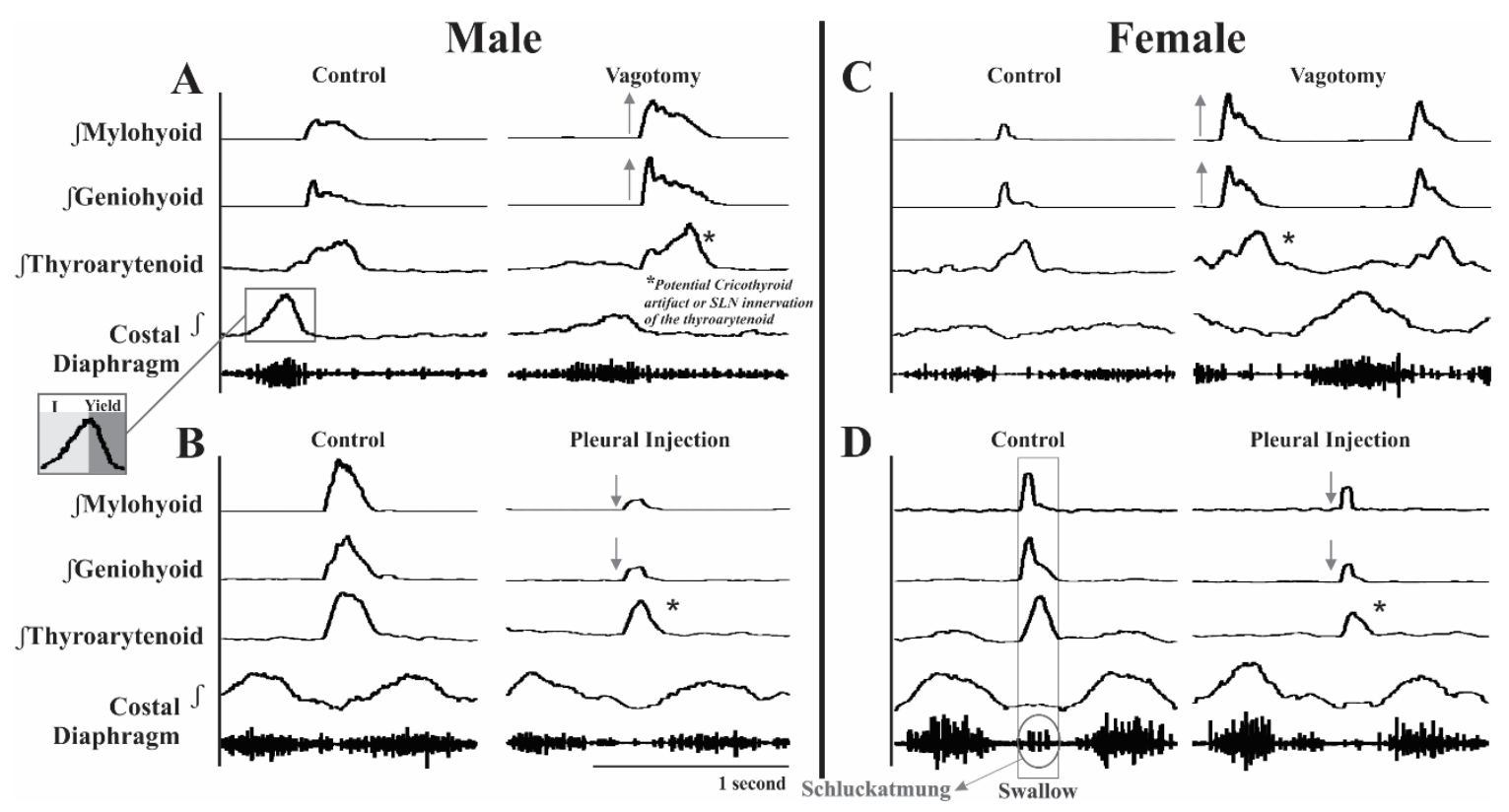

Panels A and B are recordings showing swallows from male animals. Panels C and D are recordings from female animals. In both male and female animals, upper airway amplitude increased after vagotomy (A and C) and decreased after pleural injection (B and D). Panel A demonstrates the inspiratory and yield, remnant diaphragm activity in early expiration, components of breathing. Panel D displays schluckatmung (swallow breath), diaphragm activation during swallow. 
Figure 5-2. Experimental perturbations shifted swallow-breathing coordination in females.
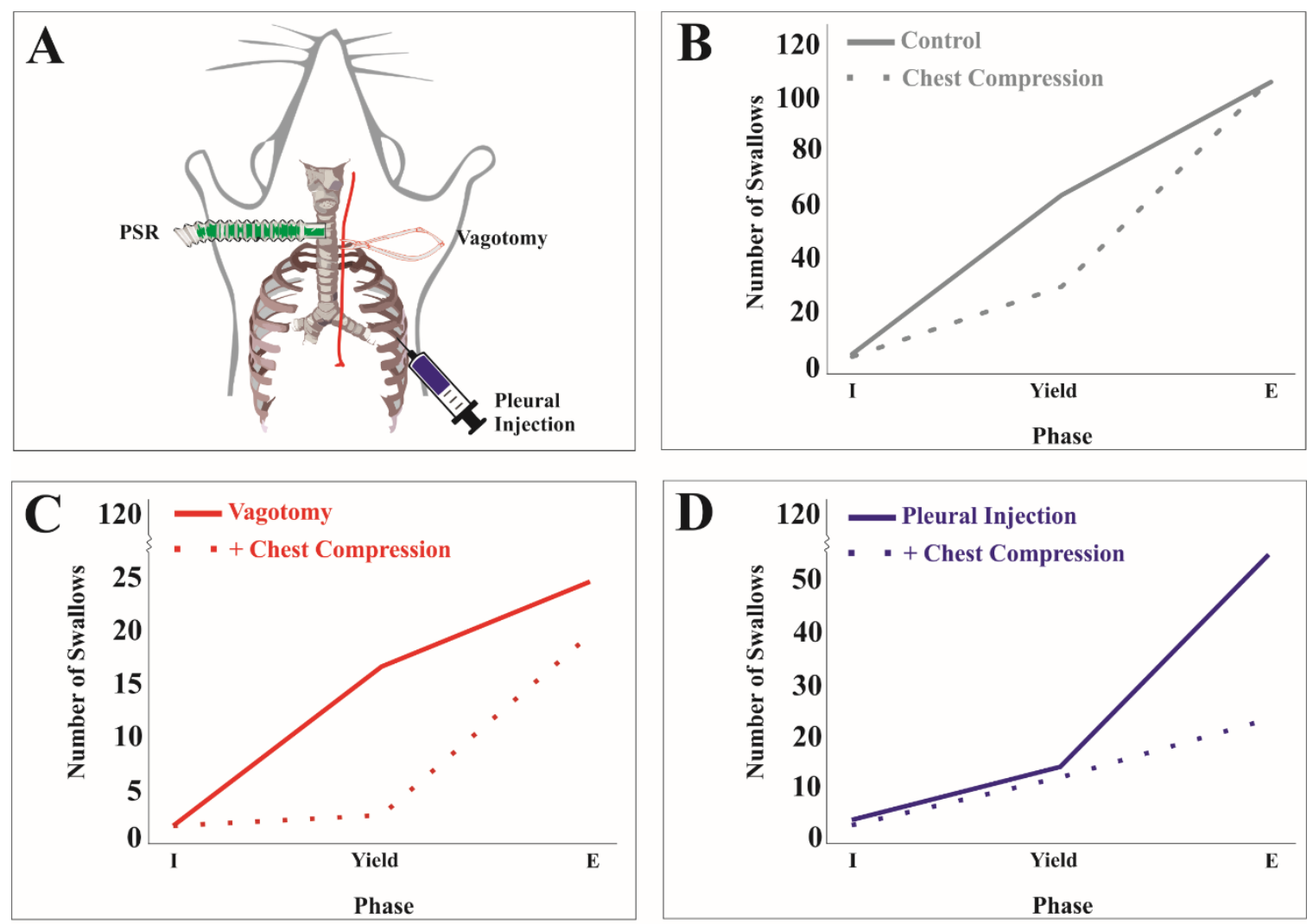

A) Illustrated representation of experimental protocol for afferent feedback manipulation.

B) Chest compression shifted swallow breathing coordination toward expiration, with swallow predominately occurring during expiration. In vagotomized females C) as well as those with reduced spinal feedback D) swallow breathing coordination shifted towards expiration when chest compression was applied. 


\section{CHAPTER 6}

\section{DISCUSSION}

These series of studies, further exploring breathing, swallow and the coordination of airway protective behaviors, has concluded with four main points. Swallow and breathing maintain coordination by using one or both strategies: phase preference and/or volume targeting. Swallow is a stable behavior and does not alter until airway patency is at risk. A balance of vagal and spinal feedback is important in maintaining swallow, breathing and swallow breathing coordination. There are breathing and swallow-related sex differences that need to be recognized for future study.

\section{Phase Preference versus Volume Targeting}

Pitts et al. (2013b) developed an aspiration protocol that resulted in the introduction of phase preference in the cat model. This stated that cats preferentially swallow during the E2 (late expiration) phase of breathing. Chapter Two transformed the aspiration cough swallow protocol from cat (Pitts, Rose, Mortensen, Poliacek, Sapienza, Lindsey, Morris, Davenport, \& Bolser, 2013b) into the human model using cough to challenge the system to move through a wide range of lung volumes and still safely swallow (Huff, Reed, Smith, Brown, Ovechkin, \& Pitts, 2018b). This is where volume targeting developed. Instead of preferentially swallowing during the expiration phase, like the cat model, the human model swallows during a specific, or targeted, lung 
volume. Humans are willing to swallow in any phase of breathing as long as a certain lung volume is maintained.

Chapter Three challenged swallow to different environmental factors allowing evaluation of swallow breathing coordination at increasing altitudes (Huff, Day, English, Reed, Zouboules, Saran, Leacy, Mann, Peltonen, \& O’Halloran, 2018). While respiratory drive increased as the respiratory system changed/adapted to hypoxia and Hypocapnia, swallow breathing coordination maintained. This study did not measure lung volume therefore; we cannot definitively say swallow occurred within the restricted lung volume found in chapter two. However, spontaneous saliva or water induced swallows did occur during all phases of breathing at each increasing altitudes.

Chapter Five manipulated lung volume by mechanically as well as systematically altering volume related sensory feedback. Chapter Two found swallow was inhibited at low and high lung volumes (Huff, Reed, Smith, Brown, Ovechkin, \& Pitts, 2018b) which led to the mechanical restriction (chest compression) protocol in chapter Five. Mechanical restriction of the thoracic cavity forced the lungs to operate at low lung volumes. Chest compression resulted in female rat swallow breathing coordination to shift to expiration regardless if vagal feedback was removed or spinal feedback reduced. However, swallow breathing coordination of the male rat was unaffected by chest compression, but altered by selectively anesthetizing PSRs shifting swallow toward inspiration occurring predominantly during yield phase. Since we did not directly measure lung volume we cannot say rodents use volume targeting to maintain swallow breathing coordination, however our data suggest in male rodents PSR volume-related feedback is necessary. We believe both phase preference and volume targeting strategies 
are necessary in maintaining swallow breathing coordination in both male and female rodents.

\section{Stability of Swallow}

Challenging cough and swallow to occur simultaneously, in chapter two, gave insight into the stability of swallow behavior. Duration of swallow and swallow related apnea, amplitude of submental complex, as well as lung volume in which swallow occurred were all unchanged when challenged with cough. Instead, cough characteristics, such as decreased inspiration and compression phases and increased number of cough epochs, were changed in order to maintain swallow breathing coordination. This suggest alteration in cough was an effort to maintain airway protection ensuring a patent airway for swallow occurrence in unstable conditions.

Both saliva and water induced swallows were not significantly altered, in chapter three, as hypoxia and hypocapnia altered cardiorespiratory conditions during ascent to altitude (Huff, Day, English, Reed, Zouboules, Saran, Leacy, Mann, Peltonen, \& O’Halloran, 2018). Submental amplitude was not altered while swallow duration did not significantly change in saliva swallows. However, post swallow apnea increased and pre swallow apnea and total swallow duration had a decreasing trend in water induced swallows as ascent to altitude increased. Environmental conditions challenged the respiratory system, swallow shifted closer to inspiration when water actively stimulated swallow. We believe the decrease in swallow duration as well as shift closer to inspiration, allowing more time before expiration, increased airway protection.

While we did not see alterations in swallow drive (amplitude increase) when challenged to coordinate with other behaviors or adapt to change in respiratory drive, 
there were alterations in amplitude when vagal and spinal feedback were removed. In chapter five removal of vagal feedback caused swallow amplitude to increase in both male and female while reduction of spinal feedback caused a reduction in swallow amplitude. We conclude that swallow does not alter motor pattern unless a high degree of afferent feedback is removed or provided.

\section{Vagal Spinal Balance}

Chapter Two concluded with the idea that humans are using enhanced pulmonary feedback to maintain cough-swallow-breathing coordination. Chapters Four and Five sought to test this theory by manipulating both vagal and spinal feedback and evaluate the response of breathing, swallow and its coordination. In swallow, we determined swallow drive is dependent upon the vagal spinal balance. In breathing, we concluded PSRs are not the primary contributor to volume feedback and diaphragm activity during yield is determined by a balance of vagal and spinal feedback.

There has been much controversy over the years discussing and interpreting the various phases of breathing: inspiration, post inspiration, E1 (early expiration), E2 (late expiration), passive versus active. Chapter Four introduced the yield phase of breathing. We believe the addition of "yield" (remnant diaphragm activity during early expiration) will distinguish and solve some debate on the difference between post inspiration, E1 and E2. Yield is the simultaneous activation of expiratory phasic laryngeal adductor (thyroarytenoid) and inspiratory phasic diaphragm activity at the start of expiration. We hypothesize yield acts to cushion forces from the chest wall on the lungs. After manipulation of vagal and spinal feedback with the addition of mechanical challenge (chest compression) we concluded there is tonic vagal inhibition on the yield phase and 
removal of vagal feedback results in disinhibition increasing yield phase. Our results suggest yield phase is spinally mediated and balance of vagal and spinal feedback is necessary to maintain this phase.

Chapter Five demonstrated the importance of vagal spinal balance on upper airway activity. Reduction in spinal feedback decreased swallow related upper airway activity, while removal of vagal feedback increased swallow related upper airway activity. This indicates that spinal feedback is excitatory toward swallow production and vagal feedback is inhibitory, much like what is seen in breathing with yield phase.

\section{Sex Differences in Breathing and Swallow Coordination}

Due to male and female anatomical differences (such as extra fat around the abdomen) we were unable to record clean sEMG signals and coordination of coughswallow-breathing (chapter Two) was only evaluated in male participants. Both male and female swallow breathing coordination at altitude was evaluated, due to low numbers in each group, both male and female data was evaluated together.

Chapter Four provided insight into sex differences in breathing characteristics not previously considered. Under control conditions, females have a longer expiration, thyroarytenoid, cycle duration and RR than males. Removal of vagal feedback heightens this phenomenon with further increase in expiratory duration, slowing RR while not have an effect on males. Females also had on average $200 \%$ greater variability than males in breathing parameters.

Chapter Five demonstrated sex differences in swallow breathing coordination. When PSRs were selectively removed, swallows occurred predominately during the yield phase of breathing in males only. When mechanical challenge was presented with and 
without perturbation of spinal and/or vagal feedback, swallow breathing coordination shifted to occurring more predominantly during expiration in females only. These results suggest, male rodents rely on volume feedback via PSRs where as female rodents rely on volume feedback via chest wall proprioception.

The large increase in variability of breathing parameters between male and female may be attributed to female animals having the smallest and largest weights of all animals in this study. Others factors such as estrus cycle could potentially effect variability though we did not study this. The sex difference in response to chest compression could be due to difference in thoracic geometry, chest wall compliance, or the size of the compression band relative to the size of the animal's thoracic cavity. Male and female rats have differences in alveolar size as well as lung volume to body mass ratio (Carey, Card, Voltz, Arbes, Germolec, Korach, \& Zeldin, 2007; Massaro, Mortola, \& Massaro, 1995). Inspiratory intercostal muscles have a greater contribution to breathing in females where as diaphragm activity is the predominate contributor in males (Bellemare, Jeanneret, \& Couture, 2003; Hutchinson, 1846; LoMauro \& Aliverti, 2018).

\section{Clincial Significance}

We believe the aspiration protocol developed in Chapter Two could potentially detect pathologic changes in airway protection in humans. Further investigation into airway coordination mechanisms using this protocol could provide important clinical understandings. Development of the swallow breathing protocol at altitude in chapter three could further be used to study swallow breathing coordination in individuals who develop high altitude pulmonary edema (HAPE) leading to pneumonia. These studies could define mechanisms useful to those who perish from aspiration pneumonia. 
Knowledge from Chapters Four and Five, that spinally mediated proprioceptive feedback regulates breathing, swallow and swallow breathing coordination could develop new therapy techniques to assist breathing and swallow concerns in spinal cord injury patients.

\section{Conclusions}

In conclusion, both vagal and spinal pulmonary feedback are necessary for production of breathing, upper airway behaviors and their coordination. Analysis of both inspiratory and expiratory breathing related muscle activity is necessary to classify breathing phase. Future breathing and/or swallow studies should evaluate both male and female models.

\section{Future Directions}

Cholera toxin subunit B (CTB), a retrograde tracer, was injected into the pleural space (in the same manner as the lidocaine pleural injections) to identify the presence of afferents in the DRG with innervation in the pleural space. Due to 1) Negative controls (no CTB injected, Figure 6-1, A-C), 2) negative primary controls, 3) negative secondary controls (primary antibody not applied and/or secondary antibody not applied) and 4) selectivity of stained neurons in the fifth cervical DRG (Figure 6-1, D-F) and $6^{\text {th }}$ thoracic DRG (Figure 6-1, G-I) we confirm there is sensory innervation of the pleural space. Unfortunately we were only able to perform the immunohistochemistry in one animal. Future studies will expand these findings to a larger population classifying innervation in both male and female as well as specific sensory innervaion to the pleural space. With this preliminary knowledge we can speculate within reason that there is sensory feedback within the pleural space contributing to lung volume feedback. Seperating out male and 
female animals could clarify some of the sex differences seen in both breathing and swallow-breathing coordination.

Concurrent experiments (with Nicholas Mellen PhD) on the sagittal section rat hindbrain (SSRH) preparation (Mellen \& Funk, 2013) were preformed, but not included in the previous chapters, sought to incorporate ficitive swallow. Fictive swallow has been classified as presence of activity on the hypoglossal (XII) rootlet and absence on the cervical rootlet (Gestreau, Milano, Bianchi, \& Grélot, 1996). With these parameters, as well as previously published swallow regions (Kessler \& Jean, 1985,) we proved fictive swallow could be centrally stimulated (Figure 6-2A) by electrical stimulation ( $20 \mathrm{~Hz}, 8$ $10 \mathrm{~V}$ ) in the NTS (blue square Figure 6-2B) in the SSRH preparation. We simultaneously stimulated ficitive swallow while optically recording calcium imaging (Figure 6-2A) in the brainstem identifiying two locations of neuron population active during ficitive swallow and silent during breathing (Figure 6-2C). The first population of neurons (top portion of 6-2C) is the more ventral population located within and just dorsal to facial nucleus. The second population of neurons is located dorsal and slightly caudal to the facial nucleus, thought to be the intermediate reticular nucleus (Ain Summan Toor, Sun, Kumar, Le, Hildreth, Phillips, \& McMullan, 2019) also thought to be called post inspiratory complex (PiCo) (Anderson, Garcia, Baertsch, Pollak, Bloom, Wei, Rai, \& Ramirez, 2016). With this preliminary knowledge we can investigate swallow realted neuron populations, central control of swallow and how swallow is modulated by breathing. 
Figure 6-1. Presence of CTB in the cervical and thoracic DRG after pleural space CTB injections.
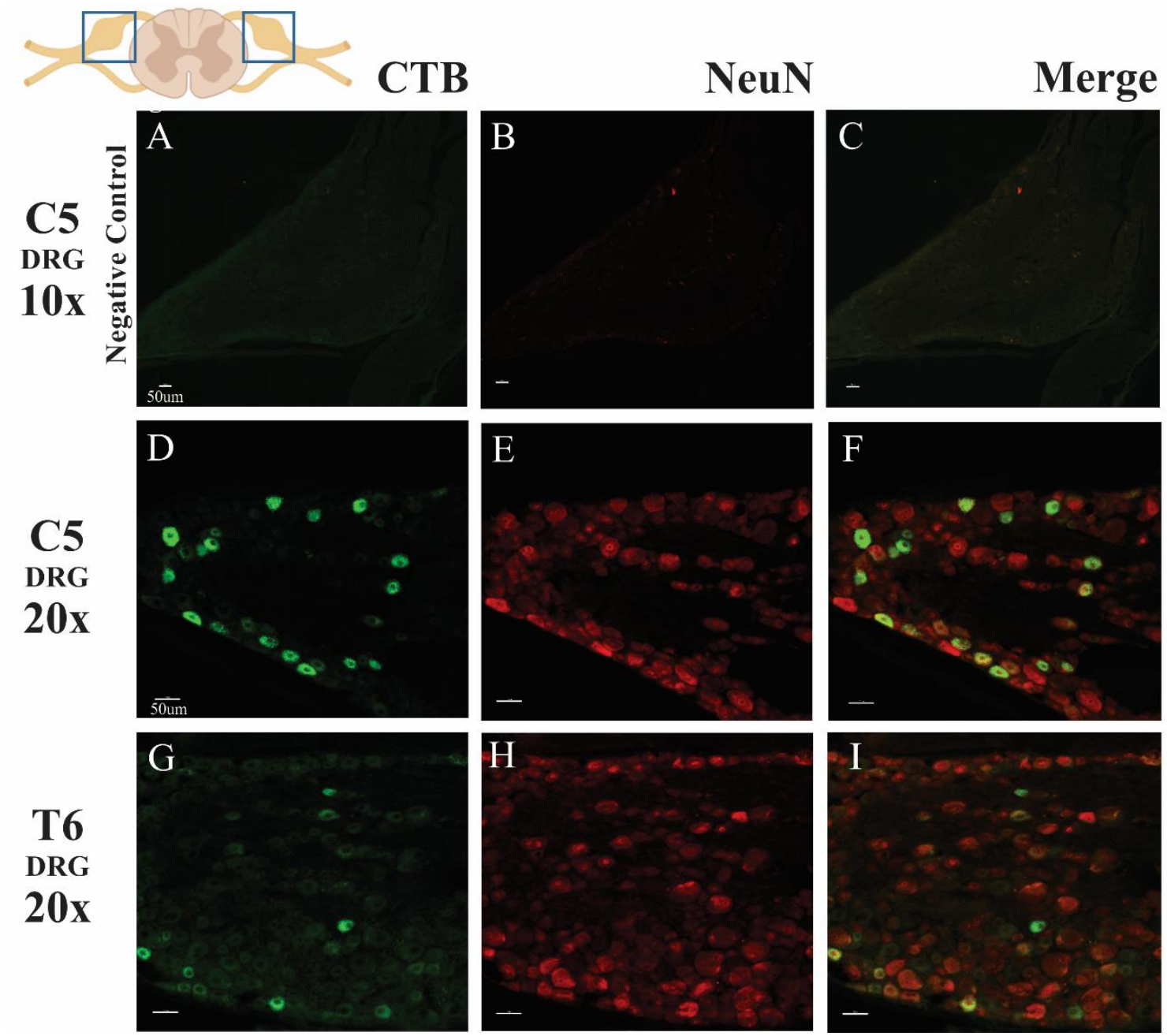

A-C) Negative control immunohistochemistry (IHC) (animals not injected with CTB) at the $5^{\text {th }}$ cervical dorsal root ganglia (DRG). (A) negative staining for cholera toxin subunit B (CTB) due to primary antibody anti-CTB was not applied and (B) negative staining due to primary antibody anti-NeuN was not applied. (C) merging of the two stains. D-F) positive labeling at the fifth cervical DRG of neurons containing CTB (D), neurons in the C5 DRG (E), and merging of both CTB and NeuN stains show the selectivity of neuons that contained CTB. G-H) shows the same results as D-F except in $6^{\text {th }}$ thoracic DRG. 
Figure 6-2. Stimulation of ficitive swallowin the sagittal section

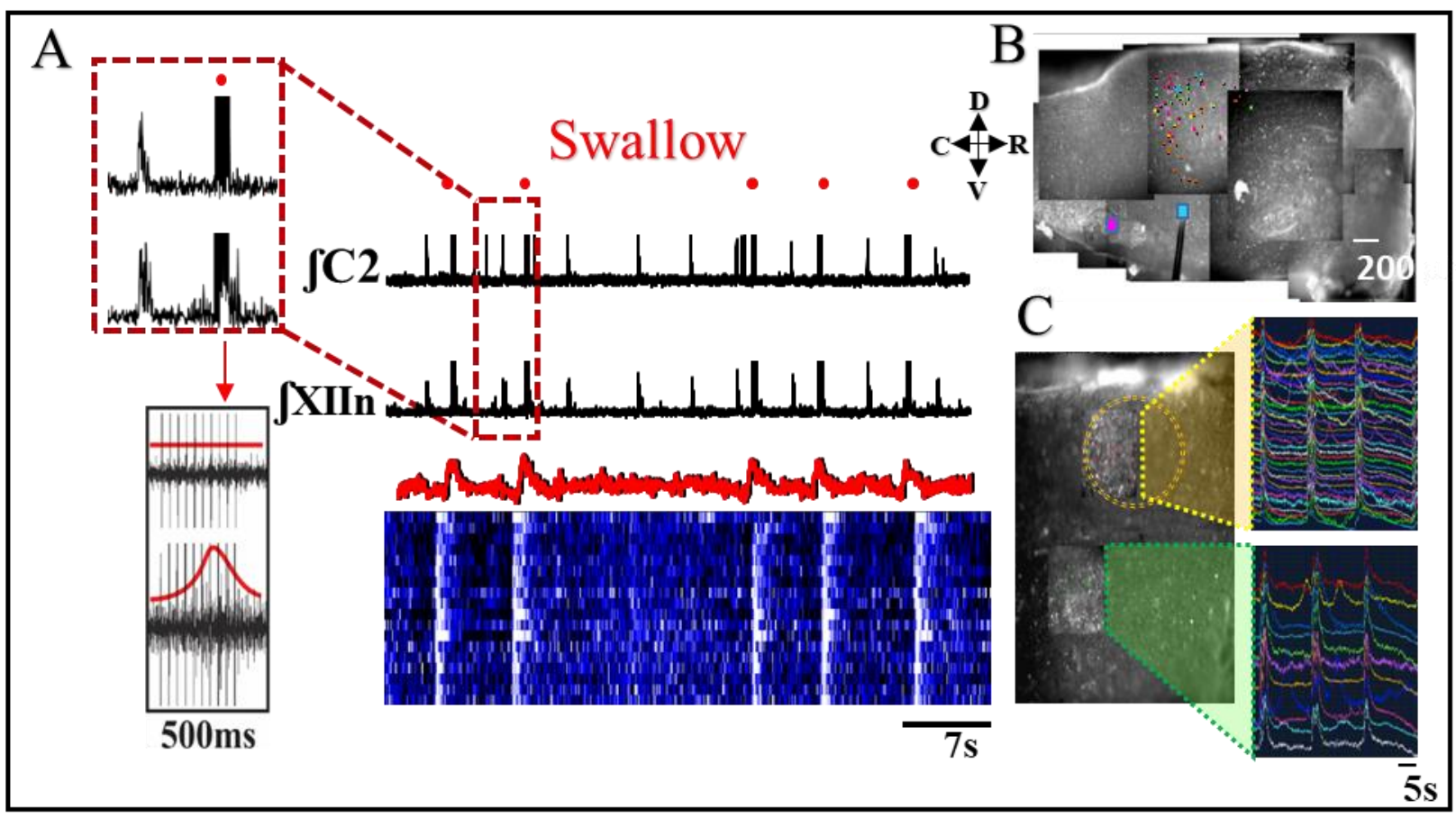

A) Representative electrophyiological trace of the second cervical rootlet (C2) and the hypoglossal (XII) rootlet. Red dots above the trace indicate electrical stimulation of swallow $(20 \mathrm{~Hz}$ at $8-10 \mathrm{~V})$. To the left in the red dashed box is a magnification of the electrical trace and below further increases magnification showing the stimulus artifact in both traces with no activity in the $\mathrm{C} 2$ root and presence of activity in the XII root. The blue image to the right of this indicates the simultaneous calcium trace of swallow related neurons activating during ficitive swallow and silence during fictive breathing. B) optical mapping image of SSRH prep with the blue square representing stimulation location for ficitive swallow and more caudual, (pink square) stimulation location for Hering-Breurer reflex (data not shown). Dorsal (D) up, ventral (V) down, Rostral (R) right, Caudal (C) left. C) the facial nucleus is circled in yellow with pink dots within corresponding to the calcium traces to the right. This is the more dorsal population of swallow related neurons. 
Below with the green dots indicate the more ventral population of neurons thought to be the intermediate reticular nucleus/PiCo. 


\section{REFERENCES}

Adrian, E. D. (1933). Afferent impulses in the vagus and their effect on respiration. $J$ Physiol, 79(3), 332-358.

Agostoni, E., D'angelo, E., Torri, G., \& Ravenna, L. (1977). Effects of uneven elastic loads on breathing pattern of anesthetized and conscious men. Respir Physiol, 30(1-2), 153-168.

Ain Summan Toor, R. U., Sun, Q.-J., Kumar, N., Le, S., Hildreth, C., Phillips, J., \& McMullan, S. (2019). Neurons in the intermediate reticular nucleus coordinate post-inspiratory activity, swallowing, and respiratory-sympathetic coupling in the rat. The Journal of Neuroscience, 0502-0519.

Anderson, T. M., Garcia, A. J., 3rd, Baertsch, N. A., Pollak, J., Bloom, J. C., Wei, A. D., Rai, K. G., \& Ramirez, J.-M. (2016). A novel excitatory network for the control of breathing. Nature, 536(7614), 76-80.

Ardran, G., \& Kemp, F. (1952). The protection of the laryngeal airway during swallowing. The British journal of radiology, 25(296), 406-416.

Askanazi, J., Silverberg, P. A., Foster, R. J., Hyman, A. I., Milic-Emili, J., \& Kinney, J. M. (1980). Effects of respiratory apparatus on breathing pattern. J Appl Physiol Respir Environ Exerc Physiol, 48(4), 577-580.

Baekey, D. M., Morris, K. F., Gestreau, C., Li, Z., Lindsey, B. G., \& Shannon, R. (2001). Medullary respiratory neurones and control of laryngeal motoneurones during fictive eupnoea and cough in the cat. J Physiol, 534(Pt 2), 565-581.

Baekey, D. M., Morris, K. F., Nuding, S. C., Segers, L. S., Lindsey, B. G., \& Shannon, R. (2003). Medullary raphe neuron activity is altered during fictive cough in the decerebrate cat. Journal of Applied Physiology, 94(1), 93-100.

Baertsch, N. A., Baertsch, H. C., \& Ramirez, J. M. (2018). The interdependence of excitation and inhibition for the control of dynamic breathing rhythms. Nature communications, 9(1), 843. 
Baertsch, N. A., Severs, L. J., Anderson, T. M., \& Ramirez, J.-M. (2019). A spatially dynamic network underlies the generation of inspiratory behaviors. Proceedings of the National Academy of Sciences, 116(15), 7493-7502.

Bailey, E. F., \& Fregosi, R. F. (2006). Modulation of upper airway muscle activities by bronchopulmonary afferents. Journal of Applied Physiology, 101(2), 609-617.

Barlow, S. M. (2009). Central pattern generation involved in oral and respiratory control for feeding in the term infant. Curr Opin Otolaryngol Head Neck Surg, 17(3), 187-193.

Bartlett, D., Jr., Sant'ambrogio, G., \& Wise, J. C. (1976). Transduction properties of tracheal stretch receptors. J Physiol, 258(2), 421-432.

Bartlett Jr, D. (1989). Respiratory functions of the larynx. Physiol Rev, 69(1), 33-57.

Bartlett Jr, D., Remmers, J., \& Gautier, H. (1973). Laryngeal regulation of respiratory airflow. Respir Physiol, 18(2), 194-204.

Basmajian, J. V., \& Stecko, G. (1962). A new bipolar electrode for electromyography. Journal of Applied Physiology, 17(5), 849-849.

Bautista, T. G., \& Dutschmann, M. (2014). Ponto-medullary nuclei involved in the generation of sequential pharyngeal swallowing and concomitant protective laryngeal adduction in situ. J Physiol, 592(12), 2605-2623.

Bautista, T. G., Sun, Q.-J., \& Pilowsky, P. M. (2014). The generation of pharyngeal phase of swallow and its coordination with breathing: Interaction between the swallow and respiratory central pattern generators Prog brain res (Vol. 212, pp. 253-275): Elsevier.

Bellemare, F., Jeanneret, A., \& Couture, J. (2003). Sex differences in thoracic dimensions and configuration. Am J Respir Crit Care Med, 168(3), 305-312.

Berger, A. J. (1977). Dorsal respiratory group neurons in the medulla of cat: Spinal projections, responses to lung inflation and superior laryngeal nerve stimulation. Brain Res, 135(2), 231-254.

Bianchi, A. L., \& Gestreau, C. (2009). The brainstem respiratory network: An overview of a half century of research. Respir Physiol Neurobiol, 168(1-2), 4-12.

Bland, S., Lazerou, L., Dyck, G., \& Cherniack, R. M. (1967). The influence of the "chest wall" on respiratory rate and depth. Respir Physiol, 3(1), 47-54.

Bolser, D., Lindsey, B., \& Shannon, R. (1983). Inhibition of medullary inspiratory drive by intercostal muscle tendon organs. Paper presented at the Soc. Neurosci. Abstr. 
Bolser, D., Lindsey, B., \& Shannon, R. (1984). Evidence that intercostal muscle-spindle endings do not reflexly modulate medullary inspiratory drive. Paper presented at the Federation Proceedings.

Bolser, D. C., Gestreau, C., Morris, K. F., Davenport, P. W., \& Pitts, T. E. (2013a). Central neural circuits for coordination of swallowing, breathing, and coughing: Predictions from computational modeling and simulation. Otolaryngol Clin North Am, 46(6), 957-964.

Bolser, D. C., Gestreau, C., Morris, K. F., Davenport, P. W., \& Pitts, T. E. (2013b). Central neural circuits for coordination of swallowing, breathing, and coughing: Predictions from computational modeling and simulation. Otolaryngol Clin North Am, 46(6), 957-964.

Bolser, D. C., Lindsey, B. G., \& Shannon, R. (1987). Medullary inspiratory activity: Influence of intercostal tendon organs and muscle spindle endings. J Appl Physiol (1985), 62(3), 1046-1056.

Bolser, D. C., Lindsey, B. G., \& Shannon, R. (1988). Respiratory pattern changes produced by intercostal muscle/rib vibration. J Appl Physiol (1985), 64(6), 24582462.

Bolser, D. C., \& Remmers, J. E. (1989). Synaptic effects of intercostal tendon organs on membrane potentials of medullary respiratory neurons. J Neurophysiol, 61(5), 918-926.

Bonham, A. (1995). Neurotransmitters in the cns control of breathing. Respir Physiol, 101(3), 219-230.

Bonis, J., Neumueller, S., Marshall, B., Krause, K., Qian, B., Pan, L., Hodges, M., \& Forster, H. (2011a). The effects of lesions in the dorsolateral pons on the coordination of swallowing and breathing in awake goats. Respiratory Physiology and Neurobiology, 175(2), 272-282.

Bonis, J. M., Neumueller, S., Marshall, B., Krause, K., Qian, B., Pan, L., Hodges, M., \& Forster, H. V. (2011b). The effects of lesions in the dorsolateral pons on the coordination of swallowing and breathing in awake goats. Respiratory physiology \& neurobiology, 175(2), 272-282.

Bonis, J. M., Neumueller, S. E., Krause, K., Pan, L., Hodges, M. R., \& Forster, H. V. (2013). Contributions of the kölliker-fuse nucleus to coordination of breathing and swallowing. Respiratory physiology \& neurobiology, 189(1), 10-21.

Bradley, G. W. (1977). Control of the breathing pattern. Int Rev Physiol, 14, 185-217. 
Bradley, G. W., von Euler, C., Marttila, I., \& Roos, B. (1975). A model of the central and reflex inhibition of inspiration in the cat. Biol Cybern, 19(2), 105-116.

Brandimore, A. E., Troche, M. S., Huber, J. E., \& Hegland, K. W. (2015). Respiratory kinematic and airflow differences between reflex and voluntary cough in healthy young adults. Frontiers in Physiology, 6, 284.

Brodsky, M. B., McFarland, D. H., Dozier, T. S., Blair, J., Ayers, C., Michel, Y., Gillespie, M. B., Day, T. A., \& Martin-Harris, B. (2010). Respiratory-swallow phase patterns and their relationship to swallowing impairment in patients treated for oropharyngeal cancer. Head Neck, 32(4), 481-489.

Bruce CD, S. G., Pfoh JR, Leacy JK, Zouboules SM, Peltonen JDB, Linares AM, Chiew AE, O'Halloran KD, Sherpa MT, Day TA. (2018). What is the point of the peak? Assessing steady-state chemoreflex drive in high altitude field studies. Adv Exp Med Biol. In Press.

Bucher, K. (1958). Pathophysiology and pharmacology of cough. Pharmacol Rev, 10(1), 43-58.

Butler, S. G., Postma, G. N., \& Fischer, E. (2004). Effects of viscosity, taste, and bolus volume on swallowing apnea duration of normal adults. Otolaryngology Head and Neck Surgery, 131(6), 860.

Campbell, E., \& Howell, J. (1962). Proprioceptive control of breathing. Pulmonary structure and function, 29-45.

Camporesi, E., Mortola, J., Sant'Ambrogio, F., \& Sant'Ambrogio, G. (1979). Topical anesthesia of tracheal receptors. Journal of Applied Physiology, 47(5), 1123-1126.

Canning, B. J., Mori, N., \& Mazzone, S. B. (2006). Vagal afferent nerves regulating the cough reflex. Respir Physiol Neurobiol, 152(3), 223-242.

Car, A. (1979). Inputs to the swallowing medullary neurons from the peripheral afferent fibers and the swallowing cortical area. Brain Res, 178(2-3), 567-572.

Car, A., Jean, A., \& Roman, C. (1975). A pontine primary relay for ascending projections of the superior laryngeal nerve. Exp Brain Res, 22(2), 197-210.

Carey, M. A., Card, J. W., Voltz, J. W., Arbes, S. J., Jr., Germolec, D. R., Korach, K. S., \& Zeldin, D. C. (2007). It's all about sex: Gender, lung development and lung disease. Trends in endocrinology and metabolism: TEM, 18(8), 308-313.

Chi-Fishman, G., \& Sonies, B. C. (2000). Motor strategy in rapid sequential swallowing: New insights. Journal of Speech, Language and Hearing Research, 43(6), 1481. 
Chiao, G., Larson, C., Yajima, Y., Ko, P., \& Kahrilas, P. J. (1994). Neuronal activity in nucleus ambiguus during deglutition and vocalization in conscious monkeys. Exp Brain Res, 100(1), 29-38.

Clark, F. J., \& von Euler, C. (1972). On the regulation of depth and rate of breathing. $J$ Physiol, 222(2), 267-295.

Clark, G. A. (1920). Deglutition apnoea. Journal of Physiology, The, 54, 59.

Cohen, M. I., \& Shaw, C.-F. (2004). Role in the inspiratory off-switch of vagal inputs to rostral pontine inspiratory-modulated neurons. Respiratory physiology \& neurobiology, 143(2-3), 127-140.

Cook, I. J., Dodds, W. J., Dantas, R. O., Kern, M. K., Massey, B. T., Shaker, R., \& Hogan, W. J. (1989). Timing of videofluoroscopic, manometric events, and bolus transit during the oral and pharyngeal phases of swallowing. Dysphagia, 4(1), 815.

Corda, M., Eklund, G., \& Von, E. (1965). External intercostal and phrenic alpha-motor responses to changes in respiratory load. Acta Physiol Scand, 63, 391-400.

Cremona, G., Asnaghi, R., Baderna, P., Brunetto, A., Brutsaert, T., Cavallaro, C., Clark, T. M., Cogo, A., Donis, R., \& Lanfranchi, P. (2002). Pulmonary extravascular fluid accumulation in recreational climbers: A prospective study. The Lancet, 359(9303), 303-309.

Cross, B. A., Guz, A., Jain, S., Archer, S., Stevens, J., \& Reynolds, F. (1976). The effect of anaesthesia of the airway in dog and man: A study of respiratory reflexes, sensations and lung mechanics. Clinical Science, 50(6), 439-454.

Culver, G. A., \& Rahn, H. (1952). Reflex respiratory stimulation by chest compression in the dog. Am J Physiol, 168(3), 686-693.

D'Angelo, E. (1982). Inspiratory muscle activity during rebreathing in intact and vagotomized rabbits. Respir Physiol, 47(2), 193-218.

D'Angelo, E., Miserocchi, G., \& Agostoni, E. (1976). Effect of rib cage or abdomen compression at iso-lung volume on breathing pattern. Respir Physiol, 28(2), 161177.

D’Angelo, O. M., Diaz-Gil, D., Nunn, D., Simons, J. C., Gianatasio, C., Mueller, N., Meyer, M. J., Pierce, E., Rosow, C., \& Eikermann, M. (2014). Anesthesia and increased hypercarbic drive impair the coordination between breathing and 
swallowing. Anesthesiology: The Journal of the American Society of Anesthesiologists, 121(6), 1175-1183.

Davenport, P. W., \& Wozniak, J. A. (1986). Effect of expiratory loading on expiratory duration and pulmonary stretch receptor discharge. Journal of Applied Physiology, 61(5), 1857-1863.

Davies, A., Dixon, M., Callanan, D., Huszczuk, A., Widdicombe, J. G., \& Wise, J. C. (1978). Lung reflexes in rabbits during pulmonary stretch receptor block by sulphur dioxide. Respir Physiol, 34(1), 83-101.

De Troyer, A. (1997). Role of joint receptors in modulation of inspiratory intercostal activity by rib motion in dogs. J Physiol, 503 ( Pt 2)(Pt 2), 445-453.

De Troyer, A., Kirkwood, P. A., \& Wilson, T. A. (2005). Respiratory action of the intercostal muscles. Physiol Rev, 85(2), 717-756.

Delcomyn, F. (1980). Neural basis of rhythmic behavior in animals. Science, 210(4469), $492-498$.

Dick, T., Oku, Y., Romaniuk, J., \& Cherniack, N. (1993a). Interaction between central pattern generators for breathing and swallowing in the cat. The Journal of Physiology, 465(1), 715.

Dick, T. E., Oku, Y., Romaniuk, J. R., \& Cherniack, N. S. (1993b). Interaction between central pattern generators for breathing and swallowing in the cat. J Physiol, 465(1), 715-730.

Dick, T. E., Shannon, R., Lindsey, B. G., Nuding, S. C., Segers, L. S., Baekey, D. M., \& Morris, K. F. (2008). Pontine respiratory-modulated activity before and after vagotomy in decerebrate cats. J Physiol, 586(17), 4265-4282.

Ding, R., Logemann, J. A., Larson, C. R., \& Rademaker, A. W. (2003). The effects of taste and consistency on swallow physiology in younger and older healthy individuals: A surface electromyographic study. Journal of Speech, Language and Hearing Research, 46(4), 977.

Dobbins, E. G., \& Feldman, J. L. (1994). Brainstem network controlling descending drive to phrenic motoneurons in rat. Journal of Comparative Neurology, 347(1), 64-86.

Dunin-Bell, O., \& Boyle, S. (2009). Secondary prevention of hape in a mount everest summiteer. High Alt Med Biol, 10(3), 293-296.

Duron, B., \& Marlot, D. (1980). The non-myelinated fibers of the phrenic and the intercostal nerves in the cat. Z Mikrosk Anat Forsch, 94(2), 257-268. 
Dutschmann, M., Bautista, T. G., Mörschel, M., \& Dick, T. E. (2014). Learning to breathe: Habituation of hering-breuer inflation reflex emerges with postnatal brainstem maturation. Respiratory physiology \& neurobiology, 195, 44-49.

Dutschmann, M., \& Dick, T. E. (2012). Pontine mechanisms of respiratory control. Comprehensive Physiology, 2(4), 2443.

Dutschmann, M., Jones, S. E., Subramanian, H. H., Stanic, D., \& Bautista, T. G. (2014). The physiological significance of postinspiration in respiratory control Prog brain res (Vol. 212, pp. 113-130): Elsevier.

Dutschmann, M., Mörschel, M., Reuter, J., Zhang, W., Gestreau, C., Stettner, G. M., \& Kron, M. (2008). Postnatal emergence of synaptic plasticity associated with dynamic adaptation of the respiratory motor pattern. Respiratory physiology \& neurobiology, 164(1-2), 72-79.

Dutschmann, M., Mörschel, M., Rybak, I. A., \& Dick, T. E. (2009). Learning to breathe: Control of the inspiratory-expiratory phase transition shifts from sensory-to central-dominated during postnatal development in rats. J Physiol, 587(20), 49314948.

Duvareille, C., Lafrance, M., Samson, N., St-Hilaire, M., Pladys, P., Micheau, P., Bournival, V., Langlois, C., \& Praud, J.-P. (2007). Effects of hypoxia and hypercapnia on nonnutritive swallowing in newborn lambs. Journal of Applied Physiology, 103(4), 1180-1188.

E, D. A., \& Agostoni, E. (1975). Tonic vagal influences on inspiratory duration. Respir Physiol, 24(3), 287-302.

Eccles, R. M., Sears, T., \& Shealy, C. (1962). Intra-cellular recording from respiratory motoneurones of the thoracic spinal cord of the cat. Nature, 193(4818), 844.

Ellenberger, H., \& Feldman, J. (1990). Brainstem connections of the rostral ventral respiratory group of the rat. Brain Res, 513(1), 35-42.

Erik Van Lunteren, K. P. S. (1988). Striated respiratory muscles of the upper airway. In G. S. A. Oommen P Mathew (Ed.), Respiratory function of the upper airway (Vol. 35, pp. 87-123). New York: Dekker.

Ertekin, C. (2014). Electrophysiological evaluation of oropharyngeal dysphagia in parkinson's disease. Journal of Movement Disorders, 7(2), 31-56.

Ezure, K., \& Tanaka, I. (2004). Gaba, in some cases together with glycine, is used as the inhibitory transmitter by pump cells in the hering-breuer reflex pathway of the rat. Neuroscience, 127(2), 409-417. 
Ezure, K., \& Tanaka, I. (2006). Distribution and medullary projection of respiratory neurons in the dorsolateral pons of the rat. Neuroscience, 141(2), 1011-1023.

Ezure, K., Tanaka, I., Saito, Y., \& Otake, K. (2002). Axonal projections of pulmonary slowly adapting receptor relay neurons in the rat. Journal of Comparative Neurology, 446(1), 81-94.

Fahim, M., \& Jain, S. (1979). The effect of bupivacaine aerosol on the activity of pulmonary stretch and'irritant'receptors. J Physiol, 288(1), 367-378.

Fay, R. A., \& Norgren, R. (1997). Identification of rat brainstem multisynaptic connections to the oral motor nuclei using pseudorabies virus: Iii. Lingual muscle motor systems. Brain research reviews, 25(3), 291-311.

Feldman, J. L. (1986). Neurophysiology of breathing in mammals. Handbook of physiology. The nervous system. intrinsic regulatory system in the brain. Am Physiol Soc, Sect, 1, 463-524.

Feroah, T. R., Forster, H., Fuentes, C. G., Lang, I. M., Beste, D., Martino, P., Pan, L., \& Rice, T. (2002). Effects of spontaneous swallows on breathing in awake goats. Journal of Applied Physiology, 92(5), 1923-1935.

Feroah, T. R., Forster, H., Fuentes, C. G., Wenninger, J., Martino, P., Hodges, M., Pan, L., \& Rice, T. (2002). Contributions from rostral medullary nuclei to coordination of swallowing and breathing in awake goats. Journal of Applied Physiology, 93(2), 581-591.

Fibla, M. S., Bernardet, U., \& Verschure, P. F. (2010). Allostatic control for robot behaviour regulation: An extension to path planning. Paper presented at the Intelligent Robots and Systems (IROS), 2010 IEEE/RSJ International Conference on.

Fitzgerald, R. S., \& Parks, D. C. (1971). Effect of hypoxia on carotid chemoreceptor response to carbon dioxide in cats. Respir Physiol, 12(2), 218-229.

Florey, H., Carleton, H., \& Wells, A. (1932). Mucus secretion in the trachea. British journal of experimental pathology, 13(3), 269.

Freer, L. (2004). Descriptive report of experience designing and staffing the first-ever medical clinic at mt. Everest base camp, 2003. High Alt Med Biol, 5(1), 89-90.

Fulwiler, C. E., \& Saper, C. B. (1984). Subnuclear organization of the efferent connections of the parabrachial nucleus in the rat. Brain research reviews, 7(3), 229-259. 
GARCIA, J. R. (1959). On the integration of respiratory movements. I. Acta physiologica latino americana, 9, 246-256.

Gautier, H. (1973). Respiratory responses of the anesthetized rabbit to vagotomy and thoracic dorsal rhizotomy. Respir Physiol, 17(2), 238-247.

German, R. Z., Crompton, A. W., \& Thexton, A. J. (2009). Integration of the reflex pharyngeal swallow into rhythmic oral activity in a neurologically intact pig model. J Neurophysiol, 102(2), 1017-1025.

Gestreau, C., Grélot, L., \& Bianchi, A. L. (2000). Activity of respiratory laryngeal motoneurons during fictive coughing and swallowing. Exp Brain Res, 130(1), 2734.

Gestreau, C., Milano, S., Bianchi, A. L., \& Grélot, L. (1996). Activity of dorsal respiratory group inspiratory neurons during laryngeal-induced fictive coughing and swallowing in decerebrate cats. Exp Brain Res, 108(2), 247-256.

Ghannouchi, I., Duclos, C., Marie, J., \& Verin, E. (2013). Modification in swallowing and ventilation co-ordination during hypercapnia, hypoxia, and tachypnea in unrestrained animals. Neurogastroenterology \& Motility, 25(4), 308.

Goslow Jr, G. E., Reinking, R. M., \& Stuart, D. G. (1973). The cat step cycle: Hind limb joint angles and muscle lengths during unrestrained locomotion. Journal of Morphology, 141(1), 1-41.

Greet, D. G., \& De Vree, F. (1984). Movements of the mandibles and tongue during mastication and swallowing in pteropus giganteus (megachiroptera): A cineradiographical study. Journal of Morphology, 179(1), 95-114.

Gürgör, N., Arıcı, Ş., Incesu, T. K., Seçil, Y., Tokuçoğlu, F., \& Ertekin, C. (2013). An electrophysiological study of the sequential water swallowing. Journal of Electromyography and Kinesiology, 23(3), 619-626.

Guyenet, P. G., \& Bayliss, D. A. (2015). Neural control of breathing and co2 homeostasis. Neuron, 87(5), 946-961.

H.M. Coleridge, J. C. G. C. (1986). Reflexes evoked from the tracheobronchial tree and lungs. In A. P. Fishman (Ed.), Handbook of physiology: The respiratory system; control of breathing (Vol. II, pp. 395-429). Bethesda, Maryland: American Physiology Society.

Hammond, C. A. S., Goldstein, L. B., Horner, R. D., Ying, J., Gray, L., Gonzalez-Rothi, L., \& Bolser, D. C. (2009). Predicting aspiration in patients with ischemic stroke: Comparison of clinical signs and aerodynamic measures of voluntary cough.

Chest, 135(3), 769-777. 
Hammouda, M., \& Wilson, W. H. (1932). The vagus influences giving rise to the phenomena accompanying expansion and collapse of the lungs. $J$ Physiol, 74(1), 81-114.

Hammouda, M., \& Wilson, W. H. (1939). Reflex acceleration of respiration arising from excitation of the vagus or its terminations in the lungs. J Physiol, 94(4), 497-524.

Hanacek, J., Davies, A., \& Widdicombe, J. G. (1984). Influence of lung stretch receptors on the cough reflex in rabbits. Respiration, 45(3), 161-168.

Harada, H., Takakusaki, K., Kita, S., Matsuda, M., Nonaka, S., \& Sakamoto, T. (2005). Effects of injecting gabaergic agents into the medullary reticular formation upon swallowing induced by the superior laryngeal nerve stimulation in decerebrate cats. Neurosci Res, 51(4), 395-404.

Hardemark Cedborg, A. I., Sundman, E., Boden, K., Hedstrom, H. W., Kuylenstierna, R., Ekberg, O., \& Eriksson, L. I. (2009). Co-ordination of spontaneous swallowing with respiratory airflow and diaphragmatic and abdominal muscle activity in healthy adult humans. Exp Physiol, 94(4), 459-468.

Harding, R. (1984). Function of the larynx in the fetus and newborn. Annu Rev Physiol, $46,645-659$.

Hashimoto, K., Sugiyama, Y., Fuse, S., Umezaki, T., Oku, Y., Dutschmann, M., \& Hirano, S. (2019). Activity of swallowing-related neurons in the medulla in the perfused brainstem preparation in rats. Laryngoscope, 129(2), E72-E79.

Hayashi, F., Coles, S. K., \& McCrimmon, D. R. (1996). Respiratory neurons mediating the breuer-hering reflex prolongation of expiration in rat. Journal of Neuroscience, 16(20), 6526-6536.

Hegland, K. W., Davenport, P. W., Brandimore, A. E., Singletary, F. F., \& Troche, M. S. (2016). Rehabilitation of swallowing and cough functions following stroke: An expiratory muscle strength training trial. Arch Phys Med Rehabil, 97(8), 13451351.

Hegland, K. W., Okun, M. S., \& Troche, M. S. (2014). Sequential voluntary cough and aspiration or aspiration risk in parkinson's disease. Lung, 192(4), 601-608.

Herbert, H., Moga, M. M., \& Saper, C. B. (1990). Connections of the parabrachial nucleus with the nucleus of the solitary tract and the medullary reticular formation in the rat. Journal of Comparative Neurology, 293(4), 540-580. 
Hildebrand, M. (1959). Motions of the running cheetah and horse. Journal of Mammalogy, 40(4), 481-495.

Hiss, S. G., Treole, K., \& Stuart, A. (2001). Effects of age, gender, bolus volume, and trial on swallowing apnea duration and swallow/respiratory phase relationships of normal adults. Dysphagia, 16(2), 128-135.

Horton, K.-K., Segers, L., Nuding, S., O’Connor, R., Alencar, P., Davenport, P., Lindsey, B., Morris, K., \& Gestreau, C. (2018). New insights into a decerebrate feline model of swallow-breathing coordination. The FASEB Journal, 32(1_supplement), 913.919-913.919.

Houston, C. S. (1960). Acute pulmonary edema of high altitude. New England Journal of Medicine, 263(10), 478-480.

Huang, S., Alexander, J., Grover, R., Maher, J., McCullough, R., McCullough, R., Moore, L., Sampson, J., Weil, J., \& Reeves, J. (1984). Hypocapnia and sustained hypoxia blunt ventilation on arrival at high altitude. Journal of Applied Physiology, 56(3), 602-606.

Huff, A., Day, T. A., English, M., Reed, M. D., Zouboules, S., Saran, G., Leacy, J. K., Mann, C., Peltonen, J. D., \& O'Halloran, K. D. (2018). Swallow-breathing coordination during incremental ascent to altitude. Respiratory physiology \& neurobiology.

Huff, A., Day, T. A., English, M., Reed, M. D., Zouboules, S., Saran, G., Leacy, J. K., Mann, C., Peltonen, J. D. B., O'Halloran, K. D., Sherpa, M. T., \& Pitts, T. (2018). Swallow-breathing coordination during incremental ascent to altitude. Respir Physiol Neurobiol.

Huff, A., Reed, M. D., Smith, B. K., Brown, E. H., Jr., Ovechkin, A. V., \& Pitts, T. (2018a). Strategies for the integration of cough and swallow to maintain airway protection in humans. Lung, 196(5), 601-608.

Huff, A., Reed, M. D., Smith, B. K., Brown, E. H., Ovechkin, A. V., \& Pitts, T. (2018b). Strategies for the integration of cough and swallow to maintain airway protection in humans. Lung, 1-8.

Hultgren, H. N. (1969). High altitude pulmonary edema. Biomedicine of high terrestrial elevations, 131-141.

Hutchinson, J. (1846). On the capacity of the lungs, and on the respiratory functions, with a view of establishing a precise and easy method of detecting disease by the spirometer. Medico-chirurgical transactions, 29, 137. 
Insalaco, G., Kuna, S. T., Costanza, B. M., Catania, G., Cibella, F., \& Bellia, V. (1991). Thyroarytenoid muscle activity during loaded and nonloaded breathing in adult humans. J Appl Physiol (1985), 70(6), 2410-2416.

Iscoe, S. (1998). Control of abdominal muscles. Progress in neurobiology, 56(4), 433506.

Iscoe, S. D. (1988). Central control of the upper airway. In G. S. A. Oommen P Mathew (Ed.), Respiratory function of the upper airway (Vol. 35, pp. 125-192). New York: Marcel Dekker.

Jafari, S., Prince, R. A., Kim, D. Y., \& Paydarfar, D. (2003). Sensory regulation of swallowing and airway protection: A role for the internal superior laryngeal nerve in humans. J Physiol, 550(1), 287-304.

Jaiswal, P. B., \& Davenport, P. W. (2016). Intercostal muscle motor behavior during tracheal occlusion conditioning in conscious rats. Journal of Applied Physiology, 120(7), 792-800.

Jean, A. (1984a). Control of the central swallowing program by inputs from the peripheral receptors. A review. J Auton Nerv Syst, 10(3-4), 225-233.

Jean, A. (1984b). Control of the central swallowing program by inputs from the peripheral receptors. A review. Journal of the autonomic nervous system, 10(3-4), 225-233.

Jean, A. (2001a). Brain stem control of swallowing: Neuronal network and cellular mechanisms. Physiol Rev, 81(2), 929-969.

Jean, A. (2001b). Brain stem control of swallowing: Neuronal network and cellular mechanisms. Physiological Review, 81(2), 929-969.

Jordan, D., \& Spyer, K. M. (1986). Brainstem integration of cardiovascular and pulmonary afferent activity. Prog Brain Res, 67, 295-314.

Karczewski, W. (1962). Effects of afferent vagal activity recorded on magnetic tape on the respiration of vagotomised animals. Bulletin of the Polish Academy of Sciences(10), 499-500.

Keller, C. J., \& Loeser, A. (1929). Der zentripetale lungenvagus. Z Biol, 89, 373-395.

Keller, E., Kohl, J., \& Koller, E. (1989). Location of pulmonary stretch receptors in the guinea-pig. Respir Physiol, 76(2), 149-157.

Kessler, J., \& Jean, A. (1985). Identification of the medullary swallowing regions in the rat. Exp Brain Res, 57(2), 256-263. 
Khurana, A., \& Thach, B. T. (1996). Effects of upper airway stimulation on swallowing, gasping, and autoresuscitation in hypoxic mice. J Appl Physiol (1985), 80(2), 472-477.

Kijima, M., Isono, S., \& Nishino, T. (1999). Coordination of swallowing and phases of respiration during added respiratory loads in awake subjects. Am J Respir Crit Care Med, 159(6), 1898-1902.

Kitagawa, J., Nakagawa, K., Hasegawa, M., Iwakami, T., Shingai, T., Yamada, Y., \& Iwata, K. (2009). Facilitation of reflex swallowing from the pharynx and larynx. Journal of oral science, 51(2), 167-171.

Kogo, M., Yamanishi, T., Koizumi, H., \& Matsuya, T. (2002). Swallowing-like activity elicited in vitro in neonatal rat organ attached brainstem block preparation. Brain Res, 955(1-2), 24-33.

Koller, E. A., \& Ferrer, P. (1970). Studies on the role of the lung deflation reflex. Respir Physiol, 10(2), 172-183.

Kolta, A., Lund, J., \& Rossignol, S. (1990). Modulation of activity of spindle afferents recorded in trigeminal mesencephalic nucleus of rabbit during fictive mastication. J Neurophysiol, 64(4), 1067-1076.

Konno, K., \& Mead, J. (1967). Measurement of the separate volume changes of rib cage and abdomen during breathing. J Appl Physiol, 22(3), 407-422.

Korpas, J., \& Jakus, J. (2000). The expiration reflex from the vocal folds. Acta Physiologica Hungarica, 87(3), 201-215.

Korpáš, J., \& Tomori, Z. (1979). Cough and other respiratory reflexes: S. Karger.

Kronecker, H., \& Meltzer, S. (1880). Über die vorgänge beim schlucken. Arch.f. Physiol, 446.

Kronecker, H., \& Meltzer, S. (1882). Ix. On the propagation of inhibitory excitation in the medulla oblongata. Proceedings of the Royal Society of London, 33(216-219), 27-29.

Kubin, L., Alheid, G. F., Zuperku, E. J., \& McCrimmon, D. R. (2006). Central pathways of pulmonary and lower airway vagal afferents. Journal of Applied Physiology, 101(2), 618-627.

Lahiri, S., \& DeLaney, R. (1975). Stimulus interaction in the responses of carotid body chemoreceptor single afferent fibers. Respir Physiol, 24(3), 249-266. 
Larrabee, M. G., \& Knowlton, G. C. (1946). Excitation and inhibition of phrenic motoneurones by inflation of the lungs. Am J Physiol, 147, 90-99.

Leow, L., Huckabee, M. L., Sharma, S., \& Tooley, T. (2006). The influence of taste on swallowing apnea, oral preparation time, and duration and amplitude of submental muscle contraction. Chemical senses, 32(2), 119-128.

Leshem, E., Pandey, P., Shlim, D. R., Hiramatsu, K., Sidi, Y., \& Schwartz, E. (2008). Clinical features of patients with severe altitude illness in nepal. Journal of travel medicine, 15(5), 315-322.

Leslie, P., Drinnan, M. J., Ford, G. A., \& Wilson, J. A. (2002). Swallow respiration patterns in dysphagic patients following acute stroke. Dysphagia, 17(3), 202-207.

Linoby, A. F., Nias, M. A., Ahmad, B. E., Zaki, S., Canda, R., Sariman, H., Azam, Z., \& Amat, A. (2013). Physiological responses and adaptations to exposure from moderate to extreme altitude: A case study of the youngest malaysian climber to scale mt. Everest.

LoMauro, A., \& Aliverti, A. (2018). Sex differences in respiratory function. Breathe, 14(2), 131.

López-Barneo, J., González-Rodríguez, P., Gao, L., Fernández-Agüera, M. C., Pardal, R., \& Ortega-Sáenz, P. (2016). Oxygen sensing by the carotid body: Mechanisms and role in adaptation to hypoxia. American Journal of Physiology-Cell Physiology, 310(8), C629-C642.

Lund, J. P. (2011). Chapter 15 - chew before you swallow. In J. P. Gossard, R. Dubuc, \& A. Kolta (Eds.), Prog brain res (Vol. 188, pp. 219-228): Elsevier.

Lust, R. M. (2007). Control of respiration.

Magendie, F. (1816). 1817. Précis élémentaire de physiologie, 2.

Mantilla, C. B., Zhan, W.-Z., \& Sieck, G. C. (2009). Retrograde labeling of phrenic motoneurons by intrapleural injection. Journal of neuroscience methods, 182(2), 244-249.

Marckwald, M. (1888). The movements of respiration and their innervation in the rabbit. With a supplement on the relation of respiration to deglutition, and on the question of the existence of respiratory centres in the spinal cord. Glasgow: Blackie \& Son.

MÅrnsson, I., \& Sandberg, N. (1974). Effects of surface anesthesia on deglutition in man. Laryngoscope, 84(3), 427-437. 
Martin-Harris, B. (2008). Clinical implications of respiratory-swallowing interactions. Curr Opin Otolaryngol Head Neck Surg, 16(3), 194-199.

Martin-Harris, B., Brodsky, M. B., Michel, Y., Castell, D. O., Schleicher, M., Sandidge, J., Maxwell, R., \& Blair, J. (2008). Mbs measurement tool for swallow impairment—mbsimp: Establishing a standard. Dysphagia, 23(4), 392-405.

Martin-Harris, B., Brodsky, M. B., Price, C. C., Michel, Y., \& Walters, B. (2003a). Temporal coordination of pharyngeal and laryngeal dynamics with breathing during swallowing: Single liquid swallows. J Appl Physiol (1985), 94(5), 17351743.

Martin-Harris, B., Brodsky, M. B., Price, C. C., Michel, Y., \& Walters, B. (2003b). Temporal coordination of pharyngeal and laryngeal dynamics with breathing during swallowing: Single liquid swallows. Journal of Applied Physiology, 94(5), 1735.

Martin-Harris, B., \& McFarland, D. H. (2013). Coordination of deglutition and respiration Principles of deglutition (pp. 25-34): Springer.

Martin, B., Logemann, J., Shaker, R., \& Dodds, W. (1994a). Coordination between respiration and swallowing: Respiratory phase relationships and temporal integration. Journal of Applied Physiology, 76(2), 714.

Martin, B. J., Logemann, J. A., Shaker, R., \& Dodds, W. J. (1994b). Coordination between respiration and swallowing: Respiratory phase relationships and temporal integration. J Appl Physiol (1985), 76(2), 714-723.

Massaro, D., \& Massaro, G. D. (2006). Estrogen receptor regulation of pulmonary alveolar dimensions: Alveolar sexual dimorphism in mice. American Journal of Physiology-Lung Cellular and Molecular Physiology, 290(5), L866-L870.

Massaro, G. D., Mortola, J. P., \& Massaro, D. (1995). Sexual dimorphism in the architecture of the lung's gas-exchange region. Proceedings of the National Academy of Sciences, 92(4), 1105-1107.

Mathew, O. P. (1988). Regulation of breathing pattern during feeding: Role of suck, swallow and nutrients. In F. B. S. A. Oommen P Mathew (Ed.), Respiratory function of the upper airway (Vol. 35, pp. 535-560). New York: Dekker.

McFarland, D. H., \& Lund, J. P. (1993). An investigation of the coupling between respiration, mastication, and swallowing in the awake rabbit. J Neurophysiol, 69(1), 95-108.

McFarland, D. H., Martin-Harris, B., Fortin, A. J., Humphries, K., Hill, E., \& Armeson, K. (2016). Respiratory-swallowing coordination in normal subjects: Lung volume at swallowing initiation. Respir Physiol Neurobiol, 234, 89-96. 
McNamara Jr, J. A., \& Moyers, R. E. (1973). Electromyography of the oral phase of deglutition in the rhesus monkey (macaca mulatta). Archives of oral biology, 18(8), 995-1002.

Mellen, N. M., \& Funk, G. D. (2013). The sagittally sectioned rat hindbrain preparation: Improved access to the brainstem respiratory network Multidisciplinary tools for investigating synaptic plasticity (pp. 257-268): Springer.

Miller, A. J. (1982a). Deglutition. Physiol Rev, 62(1), 129-184.

Miller, A. J. (1982b). Deglutition. Physiological Review, 62(1), 129-184.

Miller, F., \& Sherrington, C. (1915). Some observations on the bucco-pharyngeal stage of reflex deglutition in the cat. Exp Physiol, 9(2), 147-186.

Miserocchi, G., Mortola, J., \& Sant'ambrogio, G. (1973). Localization of pulmonary stretch receptors in the airways of the dog. J Physiol, 235(3), 775-782.

Miyazaki, M., Tanaka, I., \& Ezure, K. (1999). Excitatory and inhibitory synaptic inputs shape the discharge pattern of pump neurons of the nucleus tractus solitarii in the rat. Exp Brain Res, 129(2), 191-200.

Mondal, P., Abu-Hasan, M., Saha, A., Pitts, T., Rose, M., Bolser, D. C., \& Davenport, P. W. (2016). Effect of laparotomy on respiratory muscle activation pattern. Physiological reports, 4(1), e12668.

Monteau, R., \& Hilaire, G. (1991). Spinal respiratory motoneurons. Progress in neurobiology, 37(2), 83-144.

Nagami, S., Oku, Y., Yagi, N., Sato, S., Uozumi, R., Morita, S., Yamagata, Y., Kayashita, J., Tanimura, K., Sato, A., Takahashi, R., \& Muro, S. (2017). Breathing-swallowing discoordination is associated with frequent exacerbations of copd. BMJ Open Respiratory Research, 4(1), e000202.

Nail, B., Sterling, G., \& Widdicombe, J. (1972). Patterns of spontaneous and reflexlyinduced activity in phrenic and intercostal motoneurons. Exp Brain Res, 15(3), 318-332.

Nasri, S., Beizai, P., Ye, M., Sercarz, J. A., Kim, Y. M., \& Berke, G. S. (1997). Crossinnervation of the thyroarytenoid muscle by a branch from the external division of the superior laryngeal nerve. Ann Otol Rhinol Laryngol, $106(7 \mathrm{Pt} 1), 594-598$.

Negus, V. (1942). The mechanism of swallowing: SAGE Publications. 
Nishino, T., Kohchi, T., Honda, Y., Shirahata, M., \& Yonezawa, T. (1986). Differences in the effects of hypercapnia and hypoxia on the swallowing reflex in cats. BJA: British Journal of Anaesthesia, 58(8), 903-908.

Nishino, T., Yonezawa, T., \& Honda, Y. (1985). Effects of swallowing on the pattern of continuous respiration in human adults. Am Rev Respir Dis, 132(6), 1219-1222.

Nomura, S., \& Mizuno, N. (1982). Central distribution of afferent and efferent components of the glossopharyngeal nerve: An hrp study in the cat. Brain Res, 236(1), 1-13.

O'Connor, R., Segers, L. S., Morris, K. F., Nuding, S. C., Pitts, T., Bolser, D. C., Davenport, P. W., \& Lindsey, B. G. (2012). A joint computational respiratory neural network-biomechanical model for breathing and airway defensive behaviors. Front Physiol, 3, 264.

Oku, Y., \& Dick, T. E. (1992). Phase resetting of the respiratory cycle before and after unilateral pontine lesion in cat. Journal of Applied Physiology, 72(2), 721-730.

Oommen P Mathew, F. B. S. A. (1988). Laryngeal reflexes. In G. S. A. Oommen P Mathew (Ed.), Respiratory function of the upper airway (Vol. 35, pp. 259-302). New York: Dekker.

Ouahchi, Y., Letelier, C., Bon-Mardion, N., Marie, J.-P., Tardif, C., \& Verin, E. (2011). Effects of chronic aspirations on breathing pattern and ventilatory drive in vagatomized rats. Respiratory physiology \& neurobiology, 176(3), 98-102.

Paintal, A. S. (1973). Vagal sensory receptors and their reflex effects. Physiol Rev, 53(1), $159-227$.

Partridge, R. C. (1939). Respiratory accelerator action of the carotid sinus-cardiac depressor mechanism. J Physiol, 96(3), 233-239.

Paton, J. F., Deuchars, J., Li, Y. W., \& Kasparov, S. (2001). Properties of solitary tract neurones responding to peripheral arterial chemoreceptors. Neuroscience, 105(1), $231-248$.

Patten, S. (1789). An account of the good effects of mercury in two cases of impeded deglutition; to which is added an instance of the relief obtained from the same remedy in a spasmodic affection of the neck of the bladder. The London medical journal, 10(Pt 4), 356-361.

Paydarfar, D., Gilbert, R. J., Poppel, C. S., \& Nassab, P. F. (1995). Respiratory phase resetting and airflow changes induced by swallowing in humans. J Physiol, 483(1), 273-288. 
Pearson, K. G. (1995). Proprioceptive regulation of locomotion. Current Opinion in Neurobiology, 5(6), 786-791.

Pfoh, J. R., Steinback, C. D., Berg, E. R. V., Bruce, C. D., \& Day, T. A. (2017). Assessing chemoreflexes and oxygenation in the context of acute hypoxia: Implications for field studies. Respiratory physiology \& neurobiology, 246, 6775 .

Pinto, C. F., Balasubramanium, R. K., \& Acharya, V. (2017). Nasal airflow monitoring during swallowing: Evidences for respiratory-swallowing incoordination in individuals with chronic obstructive pulmonary disease. Lung India, 34(3), 247250.

Pitts, T., Bolser, D., Rosenbek, J., Troche, M., Okun, M. S., \& Sapienza, C. (2009). Impact of expiratory muscle strength training on voluntary cough and swallow function in parkinson disease. Chest, 135(5), 1301-1308.

Pitts, T., Bolser, D., Rosenbek, J., Troche, M., \& Sapienza, C. (2008). Voluntary cough production and swallow dysfunction in parkinson's disease. Dysphagia, 23(3), 297-301.

Pitts, T., Gayagoy, A., Rose, M., Poliacek, I., Condrey, J., Musslewhite, M., Shen, T., Davenport, P., \& Bolser, D. (2015a). Suppression of abdominal motor activity during swallowing in cats and humans. PLoS One, 10(5), e0128245-e0128245.

Pitts, T., Gayagoy, A. G., Rose, M. J., Poliacek, I., Condrey, J. A., Musslewhite, M. N., Shen, T. Y., Davenport, P. W., \& Bolser, D. C. (2015b). Suppression of abdominal motor activity during swallowing in cats and humans. PLoS One, 10(5), e0128245.

Pitts, T., Morris, K., Lindsey, B., Davenport, P., Poliacek, I., \& Bolser, D. (2012a). Coordination of cough and swallow in vivo and in silico. Experimental Physiology, 97(4), 469-473.

Pitts, T., Morris, K., Lindsey, B., Davenport, P., Poliacek, I., \& Bolser, D. (2012b). Coordination of cough and swallow in vivo and in silico. Exp Physiol, 97(4), 469473.

Pitts, T., Poliacek, I., Rose, M. J., Reed, M. D., Condrey, J. A., Tsai, H.-W., Zhou, G., Davenport, P. W., \& Bolser, D. C. (2018). Neurons in the dorsomedial medulla contribute to swallow pattern generation: Evidence of inspiratory activity during swallow. PLoS One, 13(7), e0199903.

Pitts, T., Rose, M. J., Mortensen, A. N., Poliacek, I., Sapienza, C. M., Lindsey, B. G., Morris, K. F., Davenport, P. W., \& Bolser, D. C. (2013a). Coordination of cough 
and swallow: A meta-behavioral response to aspiration. Respiratory physiology \& neurobiology, 189(3), 543-551.

Pitts, T., Rose, M. J., Mortensen, A. N., Poliacek, I., Sapienza, C. M., Lindsey, B. G., Morris, K. F., Davenport, P. W., \& Bolser, D. C. (2013b). Coordination of cough and swallow: A meta-behavioral response to aspiration. Respir Physiol Neurobiol, 189(3), 543-551.

Pitts, T., Rose, M. J., Mortensen, A. N., Poliacek, I., Sapienza, C. M., Lindsey, B. G., Morris, K. F., Davenport, P. W., \& Bolser, D. C. (2013c). Coordination of cough and swallow: A meta-behavioral response to aspiration. Respiratory physiology \& neurobiology.

Pitts, T., Rose, M. J., Poliacek, I., Condrey, J., Davenport, P. W., \& Bolser, D. C. (2015). Effect of laparotomy on the swallow-breathing relationship in the cat. Lung, 193(1), 129-133.

Pitts, T., Troche, M., Mann, G., Rosenbek, J., Okun, M. S., \& Sapienza, C. (2010). Using voluntary cough to detect penetration and aspiration during oropharyngeal swallowing in patients with parkinson disease. Chest, 138(6), 1426-1431.

Pitts, T., Troche, M. S., Carnaby-Mann, G., Rosenbek, J. C., Okun, M. S., \& Sapienza, C. M. (2010). Utilizing voluntary cough to detect penetration and aspiration during oropharyngeal swallowing in parkinson's disease. Chest.

Poliacek, I., Simera, M., Veternik, M., Kotmanova, Z., Pitts, T., Hanacek, J., Plevkova, J., Machac, P., Visnovcova, N., Misek, J., \& Jakus, J. (2016). The course of lung inflation alters the central pattern of tracheobronchial cough in cat-the evidence for volume feedback during cough. Respir Physiol Neurobiol, 229, 43-50.

Pratali, L., Cavana, M., Sicari, R., \& Picano, E. (2010). Frequent subclinical high-altitude pulmonary edema detected by chest sonography as ultrasound lung comets in recreational climbers. Critical care medicine, 38(9), 1818-1823.

Ramirez, J.-M., \& Baertsch, N. A. (2018). The dynamic basis of respiratory rhythm generation: One breath at a time. Annual review of neuroscience $(0)$.

Ravi, K. (1986). Distribution and location of slowly adapting pulmonary stretch receptors in the airways of cats. Journal of the autonomic nervous system, 15(3), 205-216.

Reed, M. D., English, M., English, C., Huff, A., Poliacek, I., Musselwhite, M. N., Howland, D. R., Bolser, D. C., \& Pitts, T. (2019). The role of the cerebellum in control of swallow: Evidence of inspiratory activity during swallow. Lung. 
Remmers, J. E. (1970). Inhibition of inspiratory activity by intercostal muscle afferents. Respir Physiol, 10(3), 358-383.

Remmers, J. E. (1973). Extra-segmental reflexes derived from intercostal afferents: Phrenic and laryngeal responses. J Physiol, 233(1), 45-62.

Richardson, C. A., Herbert, D. A., \& Mitchell, R. A. (1984). Modulation of pulmonary stretch receptors and airway resistance by parasympathetic efferents. $J$ Appl Physiol Respir Environ Exerc Physiol, 57(6), 1842-1849.

Richter, D., Ballantyne, D., \& Remmers, J. (1986). How is the respiratory rhythm generated? A model. Physiology, 1(3), 109-112.

Richter, D. W., \& Smith, J. C. (2014). Respiratory rhythm generation in vivo. Physiology, 29(1), 58-71.

Rikard-Bell, G. C., Bystrzycka, E. K., \& Nail, B. S. (1984). Brainstem projections to the phrenic nucleus: A hrp study in the cat. Brain Res Bull, 12(5), 469-477.

Rikard-Bell, G. C., Bystrzycka, E. K., \& Nail, B. S. (1985). The identification of brainstem neurones projecting to thoracic respiratory motoneurones in the cat as demonstrated by retrograde transport of hrp. Brain Res Bull, 14(1), 25-37.

Rujdomin, P. (1966). The electrical activity of the cricothyroid muscles of the cat. Archives internationales de physiologie et de biochimie, 74(1), 135-153.

Saito, Y., Ezure, K., \& Tanaka, I. (2002a). Difference between hypoglossal and phrenic activities during lung inflation and swallowing in the rat. J Physiol, 544(Pt 1), 183-193.

Saito, Y., Ezure, K., \& Tanaka, I. (2002b). Swallowing-related activities of respiratory and non-respiratory neurons in the nucleus of solitary tract in the rat. Journal of Physiology, 540(Pt 3), 1047-1060.

Saito, Y., Ezure, K., \& Tanaka, I. (2002c). Swallowing-related activities of respiratory and non-respiratory neurons in the nucleus of solitary tract in the rat. $J$ Physiol, 540(3), 1047-1060.

Sang, Q., \& Goyal, R. K. (2001). Swallowing reflex and brain stem neurons activated by superior laryngeal nerve stimulation in the mouse. American Journal of Physiology-Gastrointestinal and Liver Physiology, 280(2), G191-G200.

Sant'Ambrogio, G., Sant'Ambrogio, F. B., \& Davies, A. (1984). Airway receptors in cough. Bull Eur Physiopathol Respir, 20(1), 43-47. 
Sant'Ambrogio, G., Tsubone, H., \& Sant'Ambrogio, F. B. (1995). Sensory information from the upper airway: Role in the control of breathing. Respir Physiol, 102(1), 116.

Sasaki, C. T., \& Buckwalter, J. (1984). Laryngeal function. American journal of otolaryngology, 5(4), 281-291.

Schelegle, E. S., \& Green, J. F. (2001). An overview of the anatomy and physiology of slowly adapting pulmonary stretch receptors. Respir Physiol, 125(1-2), 17-31.

Schoene, R. B., Hackett, P. H., Henderson, W. R., Sage, E. H., Chow, M., Roach, R. C., Mills, W. J., \& Martin, T. R. (1986). High-altitude pulmonary edema. Jama, 256, 63-69.

Schoene, R. B., Swenson, E. R., Pizzo, C. J., Hackett, P. H., Roach, R. C., Mills Jr, W. J., Henderson Jr, W., \& Martin, T. (1988). The lung at high altitude:

Bronchoalveolar lavage in acute mountain sickness and pulmonary edema. Journal of Applied Physiology, 64(6), 2605-2613.

Sciortino, K. F., Liss, J. M., Case, J. L., Gerritsen, K. G. M., \& Katz, R. C. (2003). Effects of mechanical, cold, gustatory, and combined stimulation to the human anterior faucial pillars. Dysphagia, 18(1), 16-26.

Segers, L. S., Nuding, S. C., Dick, T. E., Shannon, R., Baekey, D. M., Solomon, I. C., Morris, K. F., \& Lindsey, B. G. (2008). Functional connectivity in the pontomedullary respiratory network. J Neurophysiol, 100(4), 1749-1769.

Shaker, R., Dodds, W. J., Dantas, R. O., Hogan, W. J., \& Arndorfer, R. C. (1990). Coordination of deglutitive glottic closure with oropharyngeal swallowing. Gastroenterology, 98(6), 1478-1484.

Shannon, R. (1975). Respiratory frequency control during external elastic loading and chest compression. Respir Physiol, 23(1), 11-22.

Shannon, R. (1977). Effects of thoracic dorsal rhizotomies on the respiratory pattern in anesthetized cats. J Appl Physiol Respir Environ Exerc Physiol, 43(1), 20-26.

Shannon, R. (1979a). Involvement of thoracic nerve afferents in the respiratory response to chest compression. Respir Physiol, 36(1), 65-76.

Shannon, R. (1979b). Involvement of thoracic nerve afferents in the respiratory response to chest compression. Respir Physiol, 36(1), 65-76.

Shannon, R. (1980). Intercostal and abdominal muscle afferent influence on medullary dorsal respiratory group neurons. Respir Physiol, 39(1), 73-94. 
Shannon, R. (1986). Reflexes from respiratory muscles and costovertebral joints. Handbook of Physiology. The Respiratory System. Control of Breathing(Part 1), 431-447.

Shannon, R., Baekey, D., Morris, K., Li, Z., \& Lindsey, B. (2000). Functional connectivity among ventrolateral medullary respiratory neurones and responses during fictive cough in the cat. $J$ Physiol, 525(1), 207-224.

Shannon, R., Baekey, D., Morris, K., \& Lindsey, B. (1996). Brainstem respiratory networks and cough. Pulmonary pharmacology, 9(5-6), 343-347.

Shannon, R., Baekey, D., Morris, K., Nuding, S., Segers, L., \& Lindsey, B. (2004a). Production of reflex cough by brainstem respiratory networks. Pulmonary pharmacology \& therapeutics, 17(6), 369-376.

Shannon, R., Baekey, D. M., Morris, K. F., \& Lindsey, B. G. (1998). Ventrolateral medullary respiratory network and a model of cough motor pattern generation. $J$ Appl Physiol (1985), 84(6), 2020-2035.

Shannon, R., Baekey, D. M., Morris, K. F., \& Lindsey, B. G. (2005). Central cough mechanisms: Neuroanatomy and neurophysiology. LUNG BIOLOGY IN HEALTH AND DISEASE, 205, 49.

Shannon, R., Baekey, D. M., Morris, K. F., Nuding, S. C., Segers, L. S., \& Lindsey, B. G. (2004b). Pontine respiratory group neuron discharge is altered during fictive cough in the decerebrate cat. Respiratory physiology \& neurobiology, 142(1), 4354.

Shannon, R., Bolser, D. C., \& Lindsey, B. G. (1987). Medullary expiratory activity: Influence of intercostal tendon organs and muscle spindle endings. Journal of Applied Physiology, 62(3), 1057-1062.

Shannon, R., Shear, W., Mercak, A., Bolser, D., \& Lindsey, B. (1985). Non-vagal reflex effects on medullary inspiratory neurons during inspiratory loading. Respir Physiol, 60(2), 193-204.

Shannon, R., Zechman, F., \& Frazier, D. (1972). First-breath response of medullary inspiratory neurones to the mechanical loading of inspiration. Respir Physiol, 16(1), 70-78.

Shelton Jr, R. L., Bosma, J. F., \& Sheets, B. V. (1960). Tongue, hyoid and larynx displacement in swallow and phonation. Journal of Applied Physiology, 15(2), 283-288. 
Sherrey, J. H., \& Megirian, D. (1974). Spontaneous and reflexly evoked laryngeal abductor and adductor muscle activity of cat. Exp Neurol, 43(3), 487-498.

Smith Hammond, C. A., Goldstein, L. B., Horner, R. D., Ying, J., Gray, L., GonzalezRothi, L., \& Bolser, D. C. (2009). Predicting aspiration in patients with ischemic stroke: Comparison of clinical signs and aerodynamic measures of voluntary cough. Chest, 135(3), 769-777.

Smith Hammond, C. A., Goldstein, L. B., Zajac, D. J., Gray, L., Davenport, P. W., \& Bolser, D. C. (2001). Assessment of aspiration risk in stroke patients with quantification of voluntary cough. Neurology, 56(4), 502-506.

Song, G., Xu, H., Wang, H., MacDonald, S. M., \& Poon, C.-S. (2011). Hypoxia-excited neurons in nts send axonal projections to kölliker-fuse/parabrachial complex in dorsolateral pons. Neuroscience, 175, 145-153.

Spearman, D. G., Poliacek, I., Rose, M. J., Bolser, D. C., \& Pitts, T. (2014). Variability of the pharyngeal phase of swallow in the cat. PLoS One, 9(8), e106121.

Steinback, C. D., \& Poulin, M. J. (2007). Ventilatory responses to isocapnic and poikilocapnic hypoxia in humans. Respir Physiol Neurobiol, 155(2), 104-113.

Storey, A. T. (1968). A functional analysis of sensory units innervating epiglottis and larynx.

Sumi, T. (1963a). The activity of brain-stem respiratory neurons and spinal respiratory motoneurons during swallowing. J Neurophysiol, 26(3), 466-477.

Sumi, T. (1963b). Neuronal mechanisms in swallowing. Pflüger's Archiv für die gesamte Physiologie des Menschen und der Tiere, 278(5), 467-477.

Suzuki, T., Nakazawa, K., \& Shiba, K. (2010). Swallow-related inhibition in laryngeal motoneurons. Neurosci Res, 67(4), 327-333.

Takada, M., Itoh, K., Yasui, Y., Mitani, A., Nomura, S., \& Mizuno, N. (1984). Distribution of premotor neurons for the hypoglossal nucleus in the cat. Neurosci Lett, 52(1-2), 141-146.

Tatar, M., Sant' Ambrogio, G., \& Sant' Ambrogio, F. B. (1994). Laryngeal and tracheobronchial cough in anesthetized dogs. Journal of Applied Physiology, 76(6), 2672-2679.

Taylor, A., Hidaka, O., Durbaba, R., \& Ellaway, P. H. (1997). Fusimotor influence on jaw muscle spindle activity during swallowing-related movements in the cat. $J$ Physiol, 503 ( Pt 1), 157-167. 
Thexton, A. J., Crompton, A., \& German, R. Z. (2007). Electromyographic activity during the reflex pharyngeal swallow in the pig: Doty and bosma (1956) revisited. Journal of Applied Physiology, 102(2), 587-600.

Tomori, Z., \& Widdicombe, J. (1969). Muscular, bronchomotor and cardiovascular reflexes elicited by mechanical stimulation of the respiratory tract. $J$ Physiol, 200(1), 25-49.

Trenchard, D. (1977). Role of pulmonary stretch receptors during breathing in rabbits, cats and dogs. Respir Physiol, 29(2), 231-246.

Troche, M. S., Brandimore, A. E., Godoy, J., \& Hegland, K. W. (2014). A framework for understanding shared substrates of airway protection. Journal of Applied Oral Science, 22(4), 251-260.

Troche, M. S., Brandimore, A. E., Okun, M. S., Davenport, P. W., \& Hegland, K. W. (2014). Decreased cough sensitivity and aspiration in parkinson disease. Chest, 146(5), 1294-1299.

Troche, M. S., Huebner, I., Rosenbek, J. C., Okun, M. S., \& Sapienza, C. M. (2011). Respiratory-swallowing coordination and swallowing safety in patients with parkinson's disease. Dysphagia, 26(3), 218-224.

Troche, M. S., Okun, M. S., Rosenbek, J. C., Musson, N., Fernandez, H. H., Rodriguez, R., Romrell, J., Pitts, T., Wheeler-Hegland, K. M., \& Sapienza, C. M. (2010). Aspiration and swallowing in parkinson disease and rehabilitation with emst: A randomized trial(loe classification). Neurology, 75(21), 1912-1919.

Troche, M. S., Schumann, B., Brandimore, A. E., Okun, M. S., \& Hegland, K. W. (2016). Reflex cough and disease duration as predictors of swallowing dysfunction in parkinson's disease. Dysphagia, 31(6), 757-764.

Uchida, K., Yamada, Y., \& Sato, T. (1994). The coordination of rhythmical drinking behavior with swallowing in rabbits. Physiol Behav, 55(5), 795-801.

van Lunteren, E., \& Dick, T. E. (1989). Motor unit regulation of mammalian pharyngeal dilator muscle activity. The Journal of clinical investigation, 84(2), 577-585.

van Lunteren, E., Haxhiu, M. A., \& Cherniack, N. S. (1989). Effects of tracheal airway occlusion on hyoid muscle length and upper airway volume. Journal of Applied Physiology, 67(6), 2296-2302. 
Van Oostdam, J., Walker, D., Knudson, K., Dirks, P., Dahlby, R., \& Hogg, J. (1986). Effect of breathing dry air on structure and function of airways. Journal of Applied Physiology, 61(1), 312-317.

von Euler, C., \& Peretti, G. (1966). Dynamic and static contributions to the rhythmic y activation of primary and secondary spindle endings in external intercostal muscle. J Physiol, 187(3), 501-516.

Wang, Y., \& Bieger, D. (1991). Role of solitarial gabaergic mechanisms in control of swallowing. American Journal of Physiology-Regulatory, Integrative and Comparative Physiology, 261(3), R639-R646.

Wasserman, A. M., Sahibzada, N., Hernandez, Y. M., \& Gillis, R. A. (2000). Specific subnuclei of the nucleus tractus solitarius play a role in determining the duration of inspiration in the rat. Brain Res, 880(1-2), 118-130.

Wassilieff, N. (1887). Wo wird der schluckreflex ausgelöst? , Verlag nicht ermittelbar. Weil, J. V. (1986). Ventilatory control at high altitude. Comprehensive Physiology.

Wheeler Hegland, K., Huber, J. E., Pitts, T., Davenport, P. W., \& Sapienza, C. M. (2011a). Lung volume measured during sequential swallowing in healthy young adults. Journal of Speech, Language, and Hearing Research, 54(3), 777.

Wheeler Hegland, K., Huber, J. E., Pitts, T., Davenport, P. W., \& Sapienza, C. M. (2011b). Lung volume measured during sequential swallowing in healthy young adults. J Speech Lang Hear Res, 54(3), 777-786.

Wheeler Hegland, K. M., Huber, J. E., Pitts, T., \& Sapienza, C. M. (2009a). Lung volume during swallowing: Single bolus swallows in healthy young adults. Journal of Speech, Language, and Hearing Research, 52(1), 178.

Wheeler Hegland, K. M., Huber, J. E., Pitts, T., \& Sapienza, C. M. (2009b). Lung volume during swallowing: Single bolus swallows in healthy young adults. J Speech Lang Hear Res, 52(1), 178-187.

Widdicombe, J. (1954). Respiratory reflexes from the trachea and bronchi of the cat. $J$ Physiol, 123(1), 55-70.

Widdicombe, J. (1988). Nasal and pharyngeal reflexes. In G. S. A. Oommen P Mathew (Ed.), Respiratory function of upper airway (Vol. 35, pp. 233-258). New York: Dekker.

Widdicombe, J. (2003). Functional morphology and physiology of pulmonary rapidly adapting receptors (rars). The Anatomical Record, 270(1), 2-10. 
Widdicombe, J. G. (1964). Respiratory reflexes. In H. R. W.O. Fenn (Ed.), Handbook of physiology section 3 respiration (Vol. I, pp. 585-630). Washington, D.C.: American Physiological Society.

Widdicombe, J. G. (2011). Reflexes from the upper respiratory tract. Comprehensive Physiology.

Widdicombe, J. G., \& Sellick, H. (1970). Vagal deflation and inflation reflexes mediated by lung irritant receptors. Q J Exp Physiol Cogn Med Sci, 55(2), 153-163.

Wohlgemuth, M., van der Kooi, E. L., Hendriks, J. C., Padberg, G. W., \& Folgering, H. T. (2003). Face mask spirometry and respiratory pressures in normal subjects. Eur Respir J, 22(6), 1001-1006.

Yagi, N., Oku, Y., Nagami, S., Yamagata, Y., Kayashita, J., Ishikawa, A., Domen, K., \& Takahashi, R. (2017). Inappropriate timing of swallow in the respiratory cycle causes breathing-swallowing discoordination. Front Physiol, 8, 676.

Yoshida, Y., Miyazaki, T., Hirano, M., Shin, T., Totoki, T., \& Kanaseki, T. (1980). Location of motoneurons supplying the cricopharyngeal muscle in the cat studied by means of the horseradish peroxidase method. Neurosci Lett, 18(1), 1-4.

Yoshida, Y., Miyazaki, T., Hirano, M., Shin, T., Totoki, T., \& Kanaseki, T. (1981). Localization of efferent neurons innervating the pharyngeal constrictor muscles and the cervical esophagus muscle in the cat by means of the horseradish peroxidase method. Neurosci Lett, 22(2), 91-95.

Zechman Jr, F. W., \& Wiley, R. L. (2011). Afferent inputs to breathing: Respiratory sensation. Comprehensive Physiology, 449-474. 


\section{APPENDIX A: ABBREVIATIONS}

$\% \mathrm{VC}$

$\mathrm{AB}$

Böt-VRG

C

$\mathrm{cEx}-\mathrm{cEx}$

cEx-cIn

cIn-cEx

cIn-cIn

$\mathrm{CO}_{2}$

CPD

CPG

CTB

DAPI

DRG

DSG

E

E1

E2

EBD

ECG

EE

EMG

EPPF

EPRT

Ex-Ex

Ex-In percent of vital capacity

abdomen

Bötzinger Ventral respiratory group

Cervical segment of spinal cord

expiration to expiration during cough

expiration to inspiration during cough

inspiration to expiration during cough

inspiration to inspiration during cough

Carbon Dioxide

compression phase duration

central pattern generator

Cholera Toxin subunit B

4',6-diamidino-2-phenylindole

Dorsal Root Ganglia

dorsal swallow group

expiration

early expiration

late expiration

Evans Blue Dye

Electrocardiograph

expiratory effort

electromyogram

expiratory phase peak airflow

expiratory phase rise time

expiration to expiration

expiration to inspiration 


\begin{tabular}{|c|c|}
\hline FVC & forced vital capacity \\
\hline HAPE & high altitude pulmonary edema \\
\hline HR & heart rate \\
\hline I & inspiration \\
\hline i.v. & intravenous \\
\hline IACUC & Institutional Animal Care and Use Committee \\
\hline In-Ex & inspiration to expiration \\
\hline In-In & inspiration to inspiration \\
\hline IPD & inspiratory phase duration \\
\hline IPPF & inspiratory phase peak airflow \\
\hline IPRT & inspiratory phase rise time \\
\hline IRB & Institution Review Board \\
\hline $\mathrm{L}$ & liter \\
\hline LV & lung volume \\
\hline MAP & mean atrial pressure \\
\hline $\mathrm{ms}$ & milliseconds \\
\hline NA & nucleus ambiguus \\
\hline NDS-PBS-T & normal donkey serum phosphate buffer solution triton \\
\hline $\mathrm{NeuN}$ & neuronal nuclei \\
\hline NMDA & N-methyl-D-aspartate \\
\hline NTS & nucleus tractus solitarus \\
\hline $\mathrm{O}_{2}$ & Oxygen \\
\hline PBS & phosphate buffer solution \\
\hline PCA & posterior crycoartenoid \\
\hline PD & Parkinson's disease \\
\hline $\mathrm{P}_{\mathrm{ETCO}} \mathrm{CO}_{2}$ & end tidal carboc dixocide pressure \\
\hline PSR & pulmonary stretch receptors \\
\hline
\end{tabular}




\begin{tabular}{ll} 
RC & ribcage \\
rCPG & respiratory Central Pattern Generator \\
RLN & recurrent laryngeal nerve \\
RO & right oblique \\
RR & respiratory rate \\
RTN/pFRG & retrotrapezoid nucleus/parafacial respiratory group \\
S & seconds \\
SD & standard deviation \\
SEMG & surface electromyogram \\
SI & stimulus index \\
SLN & superior laryngeal nerve \\
SpD & Sprague Dawley \\
SpO2 & peripherial oxygen saturation \\
SS-CD & inspired volume \\
T & steady state chemoreflex \\
TA & Thoracic segment of spinal cord \\
TRC & thyroarytenoid \\
UES & total respiratory cycle \\
VRG & ventral respiratory column \\
V & instantaneous minute ventilation \\
\hline
\end{tabular}




\section{APPENDIX B: LUNG LICENSE AGREEMENT}

${ }^{1}$ Confirmation Number: 11831984

Special Rightsholder Terms \& Conditions

The following terms \& conditions apply to the specific publication under which they are listed

Lung

Permission type: Republish or display content

Type of use: Thesis/Dissertation

TERMS AND CONDITIONS

The following terms are individual to this publisher:

A maximum of $10 \%$ of the content may be licensed for republication.

The user is responsible for identifying and seeking separate licenses for any third party materials that are identified anywhere in the work. Without a separate license, such third party materials may not be reused.

Other Terms and Conditions:

STANDARD TERMS AND CONDITIONS

1. Description of Service; Defined Terms. This Republication License enables the User to obtain licenses for republication of one or more copyrighted works as described in detail on the relevant Order Confirmation (the "Work(s)"). Copyright Clearance Center, Inc. ("CCC") grants licenses through the Service on behalf of the rightsholder identified on the Order Confirmation (the "Rightsholder"). "Republication", as used herein, generally means the inclusion of a Work, in whole or in part, in a new work or works, also as described on the Order Confirmation. "User", as used herein, means the person or entity making such republication.

2. The terms set forth in the relevant Order Confirmation, and any terms set by the Rightsholder with respect to a particular Work, govern the terms of use of Works in connection with the Service. By using the Service, the person transacting for a republication license on behalf of the User represents and warrants that he/she/it (a) has been duly authorized by the User to accept, and hereby does accept, all such terms and conditions on behalf of User, and (b) shall inform User of all such terms and conditions. In the event such person is a "freelancer" or other third party independent of User and CCC, such party shall be deemed jointly a "User" for purposes of these terms and conditions. In any event, User shall be deemed to have accepted and agreed to all such terms and conditions if User republishes the Work in any fashion.

3. Scope of License; Limitations and Obligations.

3.1 All Works and all rights therein, including copyright rights, remain the sole and exclusive property of the Rightsholder. The license created by the exchange of an Order Confirmation (and/or any invoice) and payment by User of the full amount set forth on that document includes only those rights expressly set forth in the Order Confirmation and in these terms and conditions, and conveys no other rights in the Work(s) to User. All rights not expressly granted are hereby reserved.

3.2 General Payment Terms: You may pay by credit card or through an account with us payable at the end of the month. If you and we agree that you may establish a standing account with CCC, then the following terms apply: Remit Payment to: Copyright Clearance Center, 29118 Network Place, Chicago, IL 60673-1291. Payments Due: Invoices are payable upon their delivery to you (or upon our notice to you that they are available to you for downloading). After 30 days, outstanding amounts will be subject to a service charge of $1-1 / 2 \%$ per month or, if less, the maximum rate allowed by applicable law. Unless otherwise specifically set forth in the Order Confirmation or in a separate written agreement signed

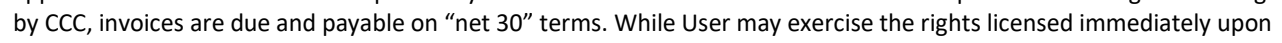
issuance of the Order Confirmation, the license is automatically revoked and is null and void, as if it had never been issued, if complete payment for the license is not received on a timely basis either from User directly or through a payment agent, such as a credit card company.

3.3 Unless otherwise provided in the Order Confirmation, any grant of rights to User (i) is "one-time" (including the editions and product family specified in the license), (ii) is non-exclusive and non-transferable and (iii) is subject to any and all limitations and restrictions (such as, but not limited to, limitations on duration of use or circulation) included in the Order Confirmation or invoice and/or in these terms and conditions. Upon completion of the licensed use, User shall either secure a new permission for further use of the Work(s) or immediately cease any new use of the Work(s) and shall render inaccessible (such as by deleting or by removing or severing links or other locators) any further copies of the Work (except for copies printed on paper in accordance with this license and still in User's stock at the end of such period). 3.4 In the event that the material for which a republication license is sought includes third party materials (such as photographs, illustrations, graphs, inserts and similar materials) which are identified in such material as having been used by permission, User is responsible for identifying, and seeking separate licenses (under this Service or otherwise) for, any of such third party materials; without a separate license, such third party materials may not be used.

3.5 Use of proper copyright notice for a Work is required as a condition of any license granted under the Service. Unless otherwise provided in the Order Confirmation, a proper copyright notice will read substantially as follows: "Republished with permission of [Rightsholder's name], from [Work's title, author, volume, edition number and year of copyright]; permission conveyed through Copyright Clearance Center, Inc. " Such notice must be provided in a reasonably legible font size and must be placed either immediately adjacent to the Work as used (for example, as part of a by-line or footnote Copyright Clearance Center https://www.copyright.com/printCoiConfirmPurchase.do?operation=defa... 3 of $67 / 15 / 2019,10: 12$ AM

but not as a separate electronic link) or in the place where substantially all other credits or notices for the new work containing the republished Work are located. Failure to include the required notice results in loss to the Rightsholder and CCC, and the User shall be liable to pay liquidated damages for each such failure equal to twice the use fee specified in 
the Order Confirmation, in addition to the use fee itself and any other fees and charges specified.

3.6 User may only make alterations to the Work if and as expressly set forth in the Order Confirmation. No Work may be used in any way that is defamatory, violates the rights of third parties (including such third parties' rights of copyright, privacy, publicity, or other tangible or intangible property), or is otherwise illegal, sexually explicit or obscene. In addition, User may not conjoin a Work with any other material that may result in damage to the reputation of the Rightsholder. User agrees to inform CCC if it becomes aware of any infringement of any rights in a Work and to cooperate with any reasonable request of CCC or the Rightsholder in connection therewith.

4. Indemnity. User hereby indemnifies and agrees to defend the Rightsholder and CCC, and their respective employees and directors, against all claims, liability, damages, costs and expenses, including legal fees and expenses, arising out of any use of a Work beyond the scope of the rights granted herein, or any use of a Work which has been altered in any unauthorized way by User, including claims of defamation or infringement of rights of copyright, publicity, privacy or other tangible or intangible property.

5. Limitation of Liability. UNDER NO CIRCUMSTANCES WILL CCC OR THE RIGHTSHOLDER BE LIABLE FOR ANY DIRECT, INDIRECT, CONSEQUENTIAL OR INCIDENTAL DAMAGES (INCLUDING WITHOUT LIMITATION DAMAGES FOR LOSS OF BUSINESS PROFITS OR INFORMATION, OR FOR BUSINESS INTERRUPTION) ARISING OUT OF THE USE OR INABILITY TO USE A WORK, EVEN IF ONE OF THEM HAS BEEN ADVISED OF THE POSSIBILITY OF SUCH DAMAGES. In any event, the total liability of the Rightsholder and CCC (including their respective employees and directors) shall not exceed the total amount actually paid by User for this license. User assumes full liability for the actions and omissions of its principals, employees, agents, affiliates, successors and assigns.

6. Limited Warranties. THE WORK(S) AND RIGHT(S) ARE PROVIDED “AS IS". CCC HAS THE RIGHT TO GRANT TO USER THE RIGHTS GRANTED IN THE ORDER CONFIRMATION DOCUMENT. CCC AND THE RIGHTSHOLDER DISCLAIM ALL OTHER WARRANTIES RELATING TO THE WORK(S) AND RIGHT(S), EITHER EXPRESS OR IMPLIED, INCLUDING WITHOUT LIMITATION IMPLIED WARRANTIES OF MERCHANTABILITY OR FITNESS FOR A PARTICULAR PURPOSE. ADDITIONAL RIGHTS MAY BE REQUIRED TO USE ILLUSTRATIONS, GRAPHS, PHOTOGRAPHS, ABSTRACTS, INSERTS OR OTHER PORTIONS OF THE WORK (AS OPPOSED TO THE ENTIRE WORK) IN A MANNER CONTEMPLATED BY USER; USER UNDERSTANDS AND AGREES THAT NEITHER CCC NOR THE RIGHTSHOLDER MAY HAVE SUCH ADDITIONAL RIGHTS TO GRANT.

7. Effect of Breach. Any failure by User to pay any amount when due, or any use by User of a Work beyond the scope of the license set forth in the Order Confirmation and/or these terms and conditions, shall be a material breach of the license created by the Order Confirmation and these terms and conditions. Any breach not cured within 30 days of written notice thereof shall result in immediate termination of such license without further notice. Any unauthorized (but licensable) use of a Work that is terminated immediately upon notice thereof may be liquidated by payment of the Rightsholder's ordinary license price therefor; any unauthorized (and unlicensable) use that is not terminated immediately for any reason (including, for example, because materials containing the Work cannot reasonably be recalled) will be subject to all remedies available at law or in equity, but in no event to a payment of less than three times the Rightsholder's ordinary license price for the most closely analogous licensable use plus Rightsholder's and/or CCC's costs and expenses incurred in collecting such payment.

8. Miscellaneous.

8.1 User acknowledges that CCC may, from time to time, make changes or additions to the Service or to these terms and conditions, and CCC reserves the right to send notice to the User by electronic mail or otherwise for the purposes of notifying User of such changes or additions; provided that any such changes or additions shall not apply to permissions already secured and paid for.

8.2 Use of User-related information collected through the Service is governed by CCC's privacy policy, available online here: http://www.copyright.com/content/cc3/en/tools/footer/privacypolicy.html.

8.3 The licensing transaction described in the Order Confirmation is personal to User. Therefore, User may not assign or transfer to any other person (whether a natural person or an organization of any kind) the license created by the Order Confirmation and these terms and conditions or any rights granted hereunder; provided, however, that User may assign such license in its entirety on written notice to CCC in the event of a transfer of all or substantially all of User's rights in the new material which includes the Work(s) licensed under this Service.

8.4 No amendment or waiver of any terms is binding unless set forth in writing and signed by the parties. The Rightsholder and CCC hereby object to any terms contained in any writing prepared by the User or its principals, employees, agents or affiliates and purporting to govern or otherwise relate to the licensing transaction described in the Order Confirmation, which terms are in any way inconsistent with any terms set forth in the Order Confirmation and/or in these terms and conditions or CCC's standard operating procedures, whether such writing is prepared prior to, simultaneously with or subsequent to the Order Confirmation, and whether such writing appears on a copy of the Order Confirmation or in a separate instrument.

8.5 The licensing transaction described in the Order Confirmation document shall be governed by and construed under the law of the State of New York, USA, without regard to the principles thereof of conflicts of law. Any case, controversy, suit, action, or proceeding arising out of, in connection with, or related to such licensing transaction shall be brought, at CCC's sole discretion, in any federal or state court located in the County of New York, State of New York, USA, or in any federal or state court whose geographical jurisdiction covers the location of the Rightsholder set forth in the Order Confirmation. The parties expressly submit to the personal jurisdiction and venue of each such federal or state court.If you have any comments or questions about the Service or Copyright Clearance Center, please contact us at 978-750-8400 or send an e-mail to info@copyright.com. v 1.1 Confirmation Number: 11831984
Citation Information

Order Detail ID: 71945642

Lung by SPRINGER-VERLAG NEW YORK. Reproduced with permission of SPRINGER-VERLAG NEW YORK in the format 


\title{
APPENDIX C: RESPIRATORY PHYSIOLOGY AND NEUROBIOLOGY LICENSE
}

\section{AGREEMENT}

\author{
${ }^{2}$ Confirmation N umber: 11831996
}

Special Rightsholder Terms \& Conditions

The following terms \& conditions apply to the specific publication under which they are listed

Respiratory physiology \& neurobiology

Permission type: Republish or display content

Type of use: Thesis/Dissertation

TERMS AND CONDITIONS

The following terms are individual to this publisher:

None

Other Terms and Conditions:

STANDARD TERMS AND CONDITIONS

1. Description of Service; Defined Terms. This Republication License enables the User to obtain licenses for republication of one or more copyrighted works as described in detail on the relevant Order Confirmation (the "Work(s)"). Copyright Clearance Center, Inc. ("CCC") grants licenses through the Service on behalf of the rightsholder identified on the Order Confirmation (the "Rightsholder"). "Republication", as used herein, generally means the inclusion of a Work, in whole or in part, in a new work or works, also as described on the Order Confirmation. "User", as used herein, means the person or entity making such republication.

2. The terms set forth in the relevant Order Confirmation, and any terms set by the Rightsholder with respect to a particular Work, govern the terms of use of Works in connection with the Service. By using the Service, the person transacting for a republication license on behalf of the User represents and warrants that he/she/it (a) has been duly authorized by the User to accept, and hereby does accept, all such terms and conditions on behalf of User, and (b) shall inform User of all such terms and conditions. In the event such person is a "freelancer" or other third party independent of User and CCC, such party shall be deemed jointly a "User" for purposes of these terms and conditions. In any event, User shall be deemed to have accepted and agreed to all such terms and conditions if User republishes the Work in any fashion.

3. Scope of License; Limitations and Obligations.

3.1 All Works and all rights therein, including copyright rights, remain the sole and exclusive property of the Rightsholder. The license created by the exchange of an Order Confirmation (and/or any invoice) and payment by User of the full amount set forth on that document includes only those rights expressly set forth in the Order Confirmation and in these terms and conditions, and conveys no other rights in the Work(s) to User. All rights not expressly granted are hereby reserved.

3.2 General Payment Terms: You may pay by credit card or through an account with us payable at the end of the month. If you and we agree that you may establish a standing account with CCC, then the following terms apply: Remit Payment to: Copyright Clearance Center, 29118 Network Place, Chicago, IL 60673-1291. Payments Due: Invoices are payable upon their delivery to you (or upon our notice to you that they are available to you for downloading). After 30 days, outstanding amounts will be subject to a service charge of 1-1/2\% per month or, if less, the maximum rate allowed by applicable law. Unless otherwise specifically set forth in the Order Confirmation or in a separate written agreement signed by CCC, invoices are due and payable on "net 30 " terms. While User may exercise the rights licensed immediately upon issuance of the Order Confirmation, the license is automatically revoked and is null and void, as if it had never been issued, if complete payment for the license is not received on a timely basis either from User directly or through a payment agent, such as a credit card company.

3.3 Unless otherwise provided in the Order Confirmation, any grant of rights to User (i) is "one-time" (including the editions and product family specified in the license), (ii) is non-exclusive and non-transferable and (iii) is subject to any and all limitations and restrictions (such as, but not limited to, limitations on duration of use or circulation) included in the Order Confirmation or invoice and/or in these terms and conditions. Upon completion of the licensed use, User shall either secure a new permission for further use of the Work(s) or immediately cease any new use of the Work(s) and shall render inaccessible (such as by deleting or by removing or severing links or other locators) any further copies of the Work (except for copies printed on paper in accordance with this license and still in User's stock at the end of such period). 3.4 In the event that the material for which a republication license is sought includes third party materials (such as photographs, illustrations, graphs, inserts and similar materials) which are identified in such material as having been used by permission, User is responsible for identifying, and seeking separate licenses (under this Service or otherwise) for, any of such third party materials; without a separate license, such third party materials may not be used.

3.5 Use of proper copyright notice for a Work is required as a condition of any license granted under the Service. Unless otherwise provided in the Order Confirmation, a proper copyright notice will read substantially as follows: "Republished with permission of [Rightsholder's name], from [Work's title, author, volume, edition number and year of copyright]; permission conveyed through Copyright Clearance Center, Inc. " Such notice must be provided in a reasonably legible font Copyright Clearance Center https://www.copyright.com/printCoiConfirmPurchase.do?operation=defa... 3 of 6 7/15/2019, 10:26 AM 
size and must be placed either immediately adjacent to the Work as used (for example, as part of a by-line or footnote but not as a separate electronic link) or in the place where substantially all other credits or notices for the new work containing the republished Work are located. Failure to include the required notice results in loss to the Rightsholder and CCC, and the User shall be liable to pay liquidated damages for each such failure equal to twice the use fee specified in the Order Confirmation, in addition to the use fee itself and any other fees and charges specified.

3.6 User may only make alterations to the Work if and as expressly set forth in the Order Confirmation. No Work may be used in any way that is defamatory, violates the rights of third parties (including such third parties' rights of copyright, privacy, publicity, or other tangible or intangible property), or is otherwise illegal, sexually explicit or obscene. In addition, User may not conjoin a Work with any other material that may result in damage to the reputation of the Rightsholder. User agrees to inform CCC if it becomes aware of any infringement of any rights in a Work and to cooperate with any reasonable request of CCC or the Rightsholder in connection therewith.

4. Indemnity. User hereby indemnifies and agrees to defend the Rightsholder and CCC, and their respective employees and directors, against all claims, liability, damages, costs and expenses, including legal fees and expenses, arising out of any use of a Work beyond the scope of the rights granted herein, or any use of a Work which has been altered in any unauthorized way by User, including claims of defamation or infringement of rights of copyright, publicity, privacy or other tangible or intangible property. 5. Limitation of Liability. UNDER NO CIRCUMSTANCES WILL CCC OR THE RIGHTSHOLDER BE LIABLE FOR ANY DIRECT, INDIRECT, CONSEQUENTIAL OR INCIDENTAL DAMAGES (INCLUDING WITHOUT LIMITATION DAMAGES FOR LOSS OF BUSINESS PROFITS OR INFORMATION, OR FOR BUSINESS INTERRUPTION) ARISING OUT OF THE USE OR INABILITY TO USE A WORK, EVEN IF ONE OF THEM HAS BEEN ADVISED OF THE POSSIBILITY OF SUCH DAMAGES. In any event, the total liability of the Rightsholder and CCC (including their respective employees and directors) shall not exceed the total amount actually paid by User for this license. User assumes full liability for the actions and omissions of its principals, employees, agents, affiliates, successors and assigns.

6. Limited Warranties. THE WORK(S) AND RIGHT(S) ARE PROVIDED “AS IS". CCC HAS THE RIGHT TO GRANT TO USER THE RIGHTS GRANTED IN THE ORDER CONFIRMATION DOCUMENT. CCC AND THE RIGHTSHOLDER DISCLAIM ALL OTHER WARRANTIES RELATING TO THE WORK(S) AND RIGHT(S), EITHER EXPRESS OR IMPLIED, INCLUDING WITHOUT LIMITATION IMPLIED WARRANTIES OF MERCHANTABILITY OR FITNESS FOR A PARTICULAR PURPOSE. ADDITIONAL RIGHTS MAY BE REQUIRED TO USE ILLUSTRATIONS, GRAPHS, PHOTOGRAPHS, ABSTRACTS, INSERTS OR OTHER PORTIONS OF THE WORK (AS OPPOSED TO THE ENTIRE WORK) IN A MANNER CONTEMPLATED BY USER; USER UNDERSTANDS AND AGREES THAT NEITHER CCC NOR THE RIGHTSHOLDER MAY HAVE SUCH ADDITIONAL RIGHTS TO GRANT.

7. Effect of Breach. Any failure by User to pay any amount when due, or any use by User of a Work beyond the scope of the license set forth in the Order Confirmation and/or these terms and conditions, shall be a material breach of the license created by the Order Confirmation and these terms and conditions. Any breach not cured within 30 days of written notice thereof shall result in immediate termination of such license without further notice. Any unauthorized (but licensable) use of a Work that is terminated immediately upon notice thereof may be liquidated by payment of the Rightsholder's ordinary license price therefor; any unauthorized (and unlicensable) use that is not terminated immediately for any reason (including, for example, because materials containing the Work cannot reasonably be recalled) will be subject to all remedies available at law or in equity, but in no event to a payment of less than three times the Rightsholder's ordinary license price for the most closely analogous licensable use plus Rightsholder's and/or CCC's costs and expenses incurred in collecting such payment.

8. Miscellaneous.

8.1 User acknowledges that CCC may, from time to time, make changes or additions to the Service or to these terms and conditions, and CCC reserves the right to send notice to the User by electronic mail or otherwise for the purposes of notifying User of such changes or additions; provided that any such changes or additions shall not apply to permissions already secured and paid for.

8.2 Use of User-related information collected through the Service is governed by CCC's privacy policy, available online here: http://www.copyright.com/content/cc3/en/tools/footer/privacypolicy.html.

8.3 The licensing transaction described in the Order Confirmation is personal to User. Therefore, User may not assign or transfer to any other person (whether a natural person or an organization of any kind) the license created by the Order Confirmation and these terms and conditions or any rights granted hereunder; provided, however, that User may assign such license in its entirety on written notice to CCC in the event of a transfer of all or substantially all of User's rights in the new material which includes the Work(s) licensed under this Service.

8.4 No amendment or waiver of any terms is binding unless set forth in writing and signed by the parties. The Rightsholder and CCC hereby object to any terms contained in any writing prepared by the User or its principals, employees, agents or affiliates and purporting to govern or otherwise relate to the licensing transaction described in the Order Confirmation, which terms are in any way inconsistent with any terms set forth in the Order Confirmation and/or in these terms and conditions or CCC's standard operating procedures, whether such writing is prepared prior to,

simultaneously with or subsequent to the Order Confirmation, and whether such writing appears on a copy of the Order Confirmation or in a separate instrument.

8.5 The licensing transaction described in the Order Confirmation document shall be governed by and construed under the law of the State of New York, USA, without regard to the principles thereof of conflicts of law. Any case, controversy, suit, action, or proceeding arising out of, in connection with, or related to such licensing transaction shall be brought, at CCC's sole discretion, in any federal or state court located in the County of New York, State of New York, USA, or in any federal 
or state court whose geographical jurisdiction covers the location of the Rightsholder set forth in the Order Confirmation. The parties expressly submit to the personal jurisdiction and venue of each such federal or state court.If you have any comments or questions about the Service or Copyright Clearance Center, please contact us at $978-750-8400$ or send an e-mail to info@copyright.com.

v 1.1

Confirmation Number: 11831996

Citation Information

Order Detail ID: 71945663

Respiratory physiology \& neurobiology by ELSEVIER BV. Reproduced with permission of ELSEVIER BV in the

format Thesis/Dissertation via Copyright Clearance Center. 


\section{CURRICULUM VITAE}

NAME:

ADDRESS:

DOB:

EDUCATION \& TRAINING:
Alyssa Danielle Huff

Department of Physiology - Kentucky Spinal Cord Injury Research Center

511 South Floyd Street, University of Louisville

Louisville, KY 40202

Gadsden, Alabama - July 30, 1993

B.S, Biology and BioPhysics

Western Kentucky University

2011-2015

M.S, Physiology

University of Louisville

2015-18

Ph.D., Physiology

University of Louisville

2015-19 

2019-2021

Anne Mehus Verbon Scholarship

2017

Caroline tum Suden/Frances A Hellebrandt Professional Opportunity Award

2017

Biology Summer Undergraduate Research Experience (BSURE)

2013

PROFESSIONAL SOCIETIES:

PUBLICATIONS: $\quad$ Reed MD, English M, English C, Huff A, Poliacek I, Musselwhite MN, Howland D, Bolser D, Pitts T (2019) The Role of the Cerebellum in Control of Swallow:Evidence of Inspiratory Activity During Swallow. Lung 1-6.

Pitts T, Bordelon R, Huff A, Byrne BJ, Smith BK (2018) Cough Effectiveness and Pulmonary Hygiene Practices in Patients with Pompe Disease. Lung 1-8.

Huff A, Reed MD, Smith BK, Brown EH, Ovechkin AV, Pitts T (2018) Strategies for the Integration of Cough and Swallow to Maintain Airway Protection in Humans. Lung 1-8

Huff A, Day TA, English M, Reed MD, Zouboules S, Saran G, Leacy JK, Mann C, Peltonen JD, O'Halloran KD (2018) SwallowBreathing Coordination during Incremental Ascent to Altitude. Respiratory Physiology \& Neurobiology.

Huff A, Brown A, Smith BK, Pitts T. Mechanisms for Successful Rehabilitation of Cough in Parkinson's disease using Expiratory Muscle Strength Training. Perspectives of the ASHA Special Interest Groups SIGS 13, Vol.2(Part 3), 2017

Wang L, McKeith A, Shen C, Carter K, Huff A, McKeith R, Zhang X, Zhengxing C. (2016) Effect of Hops Beta Acids on the Survival of Unstressed- or Acid-Stress-Adapted-Listeria 
Monocyrogenes and on the Quality and Sensory Attributes of Commercially Cured Ham Slices. Journal of Food Science 82 (2):M445-M453.

\section{PRESENTATIONS: Oral Presentations}

International

Huff A, Reed M, Mian S, Iceman K, Pitts T. Vagal and spinal pulmonary afferents contribute to respiratory variability and gender-specific response to mechanical challenge. Respiratory Trainee Highlights Breakfast. Experimental Biology, Orlando, Fl: April 2019.

Huff A, Reed M, Pitts T. Role of distention in swallow phase transition. Respiratory Trainee Highlights Breakfast. Experimental Biology, San Diego, CA: April 2018.

Huff A, Greene C, Cheffer K, O'Steen W, Howland D, Pitts T. The effects of $\mathrm{C} 2$ hemisection acutely induces swallow hyperreflexia. Respiratory Trainee Highlights Breakfast. Experimental Biology, Chicago, IL: April 2017.

Huff A, Brown EH, Aslan SC, Ovechkin AV, Pitts T. The coordination of cough and swallow behaviors in humans. Respiratory Trainee Highlights Breakfast. Experimental Biology, San Diego, CA: April 2016

\section{$\underline{\text { Regional }}$}

Huff A. The interdependence of excitation and inhibition for the control of dynamic breathing rhythms Baertsch et al. KSCIRC Journal Club, Louisville, KY: May 2019

Huff A. Vagal and spinal afferents modulate breathing and swallow. KSCIRC Seminar series. Louisville, KY: April 2019.

Huff A, Reed M, Pitts T, Gourévitch B, Mellen N. Electrical stimulation of swallow and coordination with breathing recorded optically in the sagittally-sectioned rat hindbrain. Data Blitz. Neuroscience Day. Louisville, KY: April 2019.

Huff, A. Vagal and Spinal Afferents Modulate Breathing. Ohio River Control of Breathing Symposium. Lexington, Ky: January 2019 
Huff, A. Begone Heart Artifact. Ohio River Control of Breathing Symposium. Lexington, Ky: January 2019

Huff, A. Swallow-breathing coordination during ascent to Mount Everest. Kentucky Spinal Cord Injury Research Center Seminar series. Louisville, KY: May 2018.

Huff A, Reed M, Pitts T. Swallow phase transition is mediated by distention. Data Blitz. Neuroscience Day. Louisville, KY: April 2018.

Huff, A. Coordination of cough and swallow: non-disabled human population. Kentucky Spinal Cord Injury Research Center Seminar series. Louisville, KY: July 2017.

Huff, A. Techniques from the laboratory of airway protection. Louisville Science Pathways: August 2017.

\section{Abstract/Poster Presentations}

International

Huff A, Reed M, Mian S, Iceman K, Pitts T. Respiratory variability modulated by vagal and spinal pulmonary afferents. PANAM2019: Physiology without Borders Havana, Cuba: May 2019.

Huff A, Reed M, Mian S, Iceman K, Pitts T. Vagal and spinal pulmonary afferents contribute to respiratory variability and gender-specific response to mechanical challenge. Experimental Biology, Orlando, Fl: April 2019.

Huff A, Reed M, Pitts T, Gourévitch B, Mellen N. Intercalation of swallow and breathing recorded optically in the sagittallysectioned rat hindbrain. Experimental Biology, Orlando, Fl: April 2019.

Huff A, Reed M, Pitts T. Role of distention in swallow phase transition. Experimental Biology, San Diego, CA: April 2018.

Huff A, Greene C, Cheffer K, O'Steen W, Howland D, Pitts T. Cervical hemisection increases upper airway activity in breathing and swallow. The Oxford Conference, Oxford, UK: September 2017. 
Huff A, Greene C, Cheffer K, O’Steen W, Howland D, Pitts T. Effect of cervical hemisection on swallow and airway protection. 6th Mediterranean Neuroscience Society Meeting, St. Julian's, Malta: June, 2017.

Huff A, Greene C, Cheffer K, O'Steen W, Howland D, Pitts T. The effects of $\mathrm{C} 2$ hemisection on swallow function. Experimental Biology, Chicago, IL: April 2017.

Huff A. The coordination of cough and swallow behaviors in humans. Experimental Biology, San Diego, CA: April 2016.

\section{$\underline{\text { Regional }}$}

Huff A, Reed M, Pitts T, Gourévitch B, Mellen N. Electrical stimulation of swallow and coordination with breathing recorded optically in the sagittally-sectioned rat hindbrain. Neuroscience Day. Louisville, KY: April 2019.

Huff A, Reed M, Pitts T. Role of distention in swallow phase transition using videofluoroscopy. Research!Louisville. Louisville, KY: October 2018.

Huff A, Reed M, Pitts T. Swallow phase transition is mediated by distention. Data Blitz. Neuroscience Day. Louisville, KY: April 2018.

Huff A, Greene C, Cheffer K, O'Steen W, Howland D, Pitts T. The effects of C2 hemisection on swallow function. 23rd Annual Kentucky Spinal Cord and Head Injury Trust Symposium, Louisville, KY: May, 2017.

Huff A, Greene C, Cheffer K, O'Steen W, Howland D, Pitts T. The effects of C2 hemisection on swallow function. 27th Annual Louisville Neuroscience Day, Louisville, KY: April, 2017.

Huff A, Brown EH, Abbas M, Ovechkin AV, Pitts T. The effects of cough on swallow motor pattern generation 26th Annual Louisville Neuroscience Day, Louisville, KY: April, 2016.

Huff A, Carter K, Shen C. Inactivation of unstressed adapted Listeria monocytogenes in ham extract by hops beta acids. Annual BSURE Symposium, Bowling Green, KY; October 2013. 
Huff A, Carter K, Shen C. Inactivation of unstressed adapted Listeria monocytogenes in ham extract by hops beta acids.

Kentucky Academy of Science Annual meeting, Morehead, KY November 2013

BOOKS: $\quad$ Huff A, Pitts T. (2016) "Laryngeal Reflexes". Voice Disorders. Edited by Christine Sapienza, PhD and Bari Hoffman-Ruddy, PhD

INVITED

PRESENTATIONS: Huff A, Partners in Caring Medicine in Kenya: PIC MIK. Lions Club Meeting, Morganfield, KY: May 2018.. 